\title{
Search for squarks and gluinos in events with an isolated lepton, jets, and missing transverse momentum at $\sqrt{s}=13 \mathrm{TeV}$ with the ATLAS detector
}

\author{
M. Aaboud et al." \\ (ATLAS Collaboration) \\ (Received 29 August 2017; published 26 December 2017)
}

\begin{abstract}
The results of a search for squarks and gluinos in final states with an isolated electron or muon, multiple jets and large missing transverse momentum using proton-proton collision data at a center-of-mass energy of $\sqrt{s}=13 \mathrm{TeV}$ are presented. The data set used was recorded during 2015 and 2016 by the ATLAS experiment at the Large Hadron Collider and corresponds to an integrated luminosity of $36.1 \mathrm{fb}^{-1}$. No significant excess beyond the expected background is found. Exclusion limits at $95 \%$ confidence level are set in a number of supersymmetric scenarios, reaching masses up to $2.1 \mathrm{TeV}$ for gluino pair production and up to $1.25 \mathrm{TeV}$ for squark pair production.
\end{abstract}

DOI: 10.1103/PhysRevD.96.112010

\section{INTRODUCTION}

Supersymmetry (SUSY) [1-6] is a theoretical framework of physics beyond the Standard Model (SM) which predicts for each SM particle the existence of a supersymmetric partner (sparticle) differing by half a unit of spin. The partner particles of the SM fermions (quarks and leptons) are the scalar squarks $(\tilde{q})$ and sleptons $(\tilde{\ell})$. In the boson sector, the supersymmetric partner of the gluon is the fermionic gluino $(\tilde{g})$, whereas the supersymmetric partners of the Higgs (higgsinos) and the electroweak gauge bosons (winos and bino) mix to form charged mass eigenstates (charginos) and neutral mass eigenstates (neutralinos). In the minimal supersymmetric extension of the Standard Model (MSSM) [7,8] two scalar Higgs doublets along with their higgsino partners are necessary, resulting in four chargino states $\left(\tilde{\chi}_{1,2}^{ \pm}\right)$and four neutralinos $\left(\tilde{\chi}_{1,2,3,4}^{0}\right)$. SUSY addresses the SM hierarchy problem [9-12] provided that the masses of at least some of the supersymmetric particles (most notably the higgsinos, the top squarks and the gluinos) are near the $\mathrm{TeV}$ scale.

In R-parity-conserving SUSY [13], gluinos or squarks are pair produced at the Large Hadron Collider (LHC) via the strong interaction and decay either directly or via intermediate states to the lightest supersymmetric particle (LSP). The LSP, which is assumed to be the lightest neutralino $\left(\tilde{\chi}_{1}^{0}\right)$ in this paper, is stable and weakly interacting, making it a candidate for dark matter $[14,15]$.

The decay topologies targeted in this paper are largely inspired by decay chains that could be realized in the pMSSM scenario, which is a two-dimensional subspace of

*Full author list given at the end of the article.

Published by the American Physical Society under the terms of the Creative Commons Attribution 4.0 International license. Further distribution of this work must maintain attribution to the author(s) and the published article's title, journal citation, and DOI. the 19-parameter phenomenological minimal supersymmetric Standard Model (pMSSM) [16,17]. Four SUSY models with gluino or squark pair production and different decay topologies are considered. The first two models, referred to as the gluino and squark one-step models for the rest of this paper, are SUSY simplified models [18-20] in which pair-produced gluinos or squarks decay via the lightest chargino $\left(\tilde{\chi}_{1}^{ \pm}\right)$to the LSP. In the model with gluino production, the gluino decays to the lightest chargino and two SM quarks via $\tilde{g} \rightarrow q \bar{q}^{\prime} \tilde{\chi}_{1}^{ \pm}$, as illustrated in Fig. 1 (left). The gluino decay is assumed to proceed via virtual firstand second-generation squarks, hence no bottom or top quarks are produced in the simplified model. The chargino then decays to the LSP by emitting an on- or off-shell $W$ boson, $\tilde{\chi}_{1}^{ \pm} \rightarrow W^{(*) \pm} \tilde{\chi}_{1}^{0}$, depending on the available phase space. In the MSSM this decay chain is realized when the gluino decays, via a virtual squark that is the partner particle of the left-handed SM quark, to the chargino with a dominant wino component. In the squark production model, the squark decays to the chargino via $\tilde{q} \rightarrow q^{\prime} \tilde{\chi}_{1}^{ \pm}$, followed by the same chargino decay, as illustrated in Fig. 1 (middle).

The third model, referred to as the gluino two-step model for the rest of this paper, assumes gluino pair production with a subsequent decay to the chargino via $\tilde{g} \rightarrow q \bar{q}^{\prime} \tilde{\chi}^{ \pm}$. The chargino then decays via emission of an on- or off-shell $W$ boson to the second lightest neutralino according to $\tilde{\chi}^{ \pm} \rightarrow W^{ \pm} \tilde{\chi}_{2}^{0}$. In the last step of the cascade, the second lightest neutralino decays via emission of a $Z$ boson to the LSP. The decay chain of this signal model is illustrated in Fig. 1 (right). The model is used as a proxy for SUSY scenarios with many decay products in the final state. Within the MSSM, additional decay modes lead to a significant reduction in the cross section times branching fraction for this particular decay.

Finally, the fourth set of SUSY models, the pMSSM model, is selected to have a bino-dominated neutralino as 

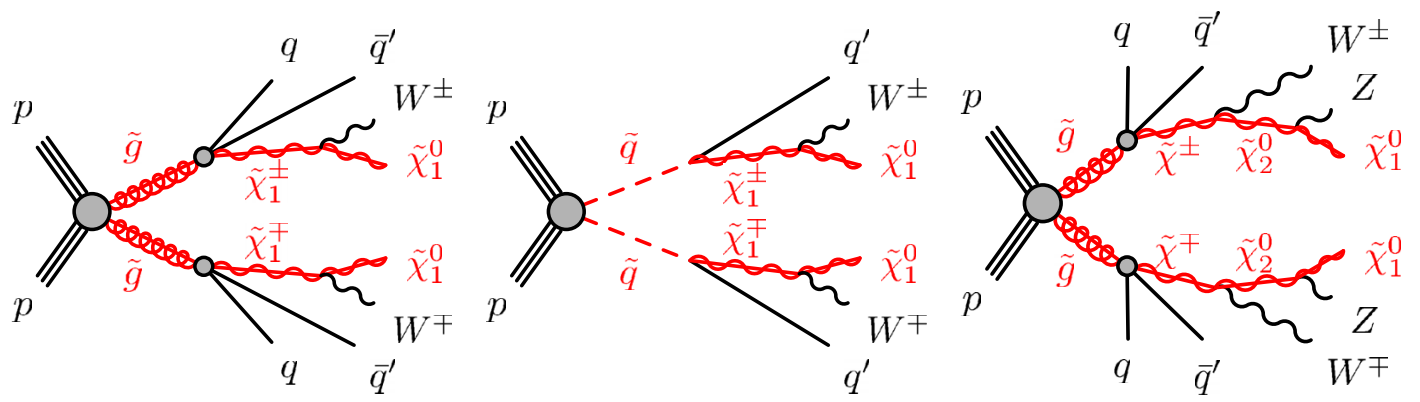

FIG. 1. The decay topologies of the simplified signal models considered in this search: gluino one-step (left), squark one-step (middle) and gluino two-step (right).

the LSP, kinematically accessible gluinos, and a higgsinodominated multiplet at intermediate mass. The higgsino multiplet contains two neutralinos (the $\tilde{\chi}_{2}^{0}$ and $\tilde{\chi}_{3}^{0}$ ) and a chargino. The decays proceed predominantly via virtual third-generation supersymmetric quarks due to their enhanced couplings with the higgsinos. Examples of dominant characteristic decay chains of this model for $m_{\tilde{\chi}_{1}^{ \pm}} \lesssim 500 \mathrm{GeV}$ and $m_{\tilde{g}} \gtrsim 1200 \mathrm{GeV}$ are $\tilde{g} \rightarrow t \bar{\tau} \tilde{\chi}_{2,3}^{0}$ and $\tilde{g} \rightarrow t \bar{b} \tilde{\chi}_{1}^{ \pm}$, with $\tilde{\chi}_{2,3}^{0}$ decaying to $Z / h \tilde{\chi}_{1}^{0}$ and $\tilde{\chi}_{1}^{ \pm}$to $W^{ \pm} \tilde{\chi}_{1}^{0}$.

In this search, the experimental signature consists of a lepton (electron or muon), several jets, and missing transverse momentum $\left(E_{\mathrm{T}}^{\text {miss }}\right)$ from the undetectable neutralinos and neutrino(s). Depending on the sparticle masses of the model considered, different amounts of energy are available in their decays. Therefore, the number of leptons and jets in the final state, as well as their kinematic properties, depend on the mass spectrum in the model of interest. Four signal regions with jet multiplicities ranging from two to six are defined to provide sensitivity to a broad range of mass spectra in the gluino and squark one-step models. For the two-step and pMSSM models, a dedicated signal region requiring nine jets is constructed to take advantage of the large jet multiplicities in these models. In each signal region, the event yield is compared with the SM prediction, which is estimated using a combination of simulation and observed data in control regions.

The search presented in this paper uses the ATLAS data collected in proton-proton collisions at the LHC during 2015 and 2016 at a center-of-mass energy of $13 \mathrm{TeV}$, corresponding to an integrated luminosity of $36.1 \mathrm{fb}^{-1}$. The analysis extends previous ATLAS searches with similar event selections performed with data collected in 20102012 (LHC Run-1) [21] and in 2015 (Run-2) [22], at center-of-mass energies of $8 \mathrm{TeV}$ and $13 \mathrm{TeV}$, respectively. Similar searches for gluinos and squarks with decays via intermediate supersymmetric particles were performed by the CMS Collaboration in Run $1[23,24]$ and Run 2 [25-27]. The results of all Run-1 ATLAS searches targeting squark and gluino pair production are summarized in Ref. [28]. The same SUSY models considered in this paper were also targeted in other Run-2 ATLAS searches using different experimental signatures [29-31].
This paper is structured as follows. After a brief description of the ATLAS detector in Sec. II, the simulated data samples for the background and signal processes used in the analysis as well as the data set and the trigger strategy are detailed in Sec. III. The reconstructed objects and quantities used in the analysis are described in Sec. IV and the event selection is presented in Sec. V. The background estimation and the systematic uncertainties associated with the expected event yields are discussed in Secs. VI and VII, respectively. Finally, the results of the analysis are presented in Sec. VIII, and are followed by a conclusion.

\section{ATLAS DETECTOR}

ATLAS [32] is a general-purpose detector with a forward-backward symmetric design that provides almost full solid angle coverage around the interaction point. ${ }^{1}$ The main components are the inner detector (ID), which is surrounded by a superconducting solenoid providing a $2 \mathrm{~T}$ axial magnetic field, the calorimeter system, and the muon spectrometer (MS), which is immersed in a magnetic field generated by three large superconducting toroidal magnets. The ID provides track reconstruction within $|\eta|<2.5$, employing pixel detectors close to the beam pipe, silicon microstrip detectors at intermediate radii, and a straw-tube tracker with particle identification capabilities based on transition radiation at radii up to $1080 \mathrm{~mm}$. The innermost pixel detector layer, the insertable B-layer [33], was added during the shutdown between LHC Run 1 and Run 2, at a radius of $33 \mathrm{~mm}$ around a new, narrower, beam pipe. The calorimeters cover $|\eta|<4$.9. The forward region $(3.2<|\eta|<4.9)$ is instrumented with a liquid-argon (LAr) calorimeter for both the electromagnetic and

\footnotetext{
${ }^{1}$ ATLAS uses a right-handed coordinate system with its origin at the nominal interaction point (IP) in the center of the detector and the $z$ axis along the beam pipe. The $x$ axis points from the IP to the center of the LHC ring, and the $y$ axis points upward. Cylindrical coordinates $(r, \phi)$ are used in the transverse plane, $\phi$ being the azimuthal angle around the $z$ axis. The pseudorapidity is defined in terms of the polar angle $\theta$ as $\eta=-\ln \tan (\theta / 2)$. Rapidity is defined as $y=0.5 \ln \left[\left(E+p_{z}\right) /\left(E-p_{z}\right)\right]$ where $E$ denotes the energy and $p_{z}$ is the component of the momentum along the beam direction.
} 
hadronic measurements. In the central region, a lead/LAr electromagnetic calorimeter covers $|\eta|<3.2$, while the hadronic calorimeter uses two different detector technologies, with scintillator tiles $(|\eta|<1.7)$ or liquid argon $(1.5<|\eta|<3.2)$ as the active medium. The MS consists of three layers of precision tracking chambers providing coverage over $|\eta|<2.7$, while dedicated fast chambers allow triggering over $|\eta|<2.4$. The ATLAS trigger system used for real-time event selection [34] consists of a hardware-based first-level trigger and a software-based high-level trigger.

\section{SIMULATED EVENT SAMPLES AND DATA SAMPLES}

Three simplified SUSY signal models and a set of pMSSM scenarios are considered in this search. Gluinos or squarks are assumed to be produced in pairs $(\tilde{g} \tilde{g}$ or $\tilde{q} \overline{\tilde{q}})$. In the case of the simplified models, $100 \%$ branching ratios to the decay of interest are assumed.

The gluino/squark one-step simplified models have three free parameters: the masses of the gluino or squark $\left(m_{\tilde{g} / \tilde{q}}\right)$, the lightest chargino $\left(m_{\tilde{\chi}_{1}^{ \pm}}\right)$, and the lightest neutralino $\left(m_{\tilde{\chi}_{1}^{0}}\right)$. Other sparticles that do not appear in the decay chain are set to have a high mass. To probe a broad range of SUSY mass spectra, two model parametrizations are considered. In the first type, $m_{\tilde{g} / \tilde{q}}$ and the mass ratio $x \equiv$ $\left(m_{\tilde{\chi}_{1}^{ \pm}}-m_{\tilde{\chi}_{1}^{0}}\right) /\left(m_{\tilde{g} / \tilde{q}}-m_{\tilde{\chi}_{1}^{0}}\right)$ are free parameters, while $m_{\tilde{\chi}_{1}^{0}}$ is fixed to $60 \mathrm{GeV}$. In the second type, $m_{\tilde{g} / \tilde{q}}$ and $m_{\tilde{\chi}_{1}^{0}}$ are free parameters, while $m_{\tilde{\chi}_{1}^{ \pm}}$is fixed by setting $x=1 / 2$. For the rest of this paper, the former type is referred to as variable- $x$ and the latter one is referred to as $x=1 / 2$.

The gluino two-step simplified model has two free parameters that are varied to probe different mass configurations: the masses of the gluino $\left(m_{\tilde{g}}\right)$ and the lightest neutralino $\left(m_{\tilde{\chi}_{1}}\right)$. The masses of the lightest chargino and the second-lightest neutralino are constrained to be $m_{\tilde{\chi}_{1}^{ \pm}}=$ $\left(m_{\tilde{g}}+m_{\tilde{\chi}_{1}^{0}}\right) / 2$ and $m_{\tilde{\chi}_{2}^{0}}=\left(m_{\tilde{\chi}_{1}^{ \pm}}+m_{\tilde{\chi}_{1}^{0}}\right) / 2$, respectively. All other sparticles are kinematically inaccessible.

In the pMSSM scenario, the sparticle masses are varied by scanning the gluino mass parameter $M_{3}$ (related to $m_{\tilde{g}}$ ) and the bilinear Higgs mass parameter $\mu$ (related to $m_{\tilde{\chi}_{1}^{ \pm}}$and $\left.m_{\tilde{\chi}_{2}^{0}}\right)$. The scan ranges are $690 \mathrm{GeV}<M_{3}<2140 \mathrm{GeV}$ and $-770 \mathrm{GeV}<\mu<-160 \mathrm{GeV}$. The bino mass parameter $M_{1}$ (related to $m_{\tilde{\chi}_{1}^{0}}$ ) was set to $60 \mathrm{GeV}$. The remaining model parameters, defined in Ref. [35], are set to $M_{A}=M_{2}=3 \mathrm{TeV}, A_{\tau}=0, \tan \beta=10$, and $A_{t}=A_{b}=$ $m_{\tilde{\mathrm{L}_{\mathrm{L}(1,2,3)}}}=m_{\left(\tilde{e_{\mathrm{R}}}, \tilde{u_{\mathrm{R}}}, \tilde{\tau_{\mathrm{R}}}\right)}=m_{\tilde{Q}_{\mathrm{L}(1,2,3)}}=m_{\left(\tilde{u_{\mathrm{R}}}, \tilde{c_{\mathrm{R}}}, \tilde{t_{\mathrm{R}}}\right)}=m_{\left(\tilde{d_{\mathrm{R}}}, \tilde{\mathrm{s}_{\mathrm{R}}}, \tilde{\hat{R}_{\mathrm{R}}}\right)}=$ $5 \mathrm{TeV}$, such that the mass of the lightest Higgs boson is compatible with $125 \mathrm{GeV}$ and all other sparticles are kinematically inaccessible. Mass spectra consistent with electroweak symmetry breaking were generated using
SOFTSUSY 3.4.0 [36] and the decay branching ratios were calculated with SDECAY/HDECAY 1.3b/3.4 [37].

The signal samples were generated at leading order (LO) using MADGRAPH 2.2.2 [38] with up to two extra partons in the matrix element, interfaced to PYTHIA 8.186 [39] for parton showers and hadronization. The CKKW-L matching scheme [40] was applied for the matching of the matrix element and the parton shower, with a scale parameter set to a quarter of the mass of the sparticle produced. The ATLAS A14 [41] set of tuned parameters (tune) was used for the shower and the underlying event, together with the NNPDF2.3 LO [42] parton distribution function (PDF) set. The EvtGen 1.2.0 program [43] was used to describe the properties of the bottom and charm hadron decays in the signal samples.

The signal cross sections were calculated at next-toleading order (NLO) in the strong coupling constant, adding the resummation of soft gluon emission at nextto-leading-logarithmic accuracy (NLL) [44-48]. The nominal cross section and its uncertainty are taken from an envelope of cross section predictions using different PDF sets and factorization and renormalization scales, as described in Ref. [49], considering only the four lightflavor left-handed squarks $\left(\tilde{u}_{L}, \tilde{d}_{L}, \tilde{s}_{L}\right.$, and $\left.\tilde{c}_{L}\right)$.

The simulated event samples for the signal and SM backgrounds are summarized in Table I. Additional samples are used to assess systematic uncertainties, as explained in Sec. VII.

To generate $t \bar{t}$ and single-top-quark events in the $W t$ and $s$-channel [50], the PowHEG-BoX v2 [51] event generator with the CT10 [52] PDF set in the matrix-element calculations was used. Electroweak $t$-channel single-top-quark events were generated using the PowHEG-Box v1 event generator. This event generator uses the four-flavor scheme for the NLO matrix-element calculations together with the fixed four-flavor PDF set CT10f4. For all top quark processes, top quark spin correlations are preserved (for the single-top $t$-channel, top quarks are decayed using MADSPIN [53]). The parton shower, fragmentation, and the underlying event were simulated using PYTHIA 6.428 [54] with the CTEQ6L1 [55] PDF set and the corresponding PERUGIA2012 tune (P2012) [56]. The top quark mass was set to $172.5 \mathrm{GeV}$. The EvTGEN 1.2.0 program was also used to describe the properties of the bottom and charm hadron decays in the $t \bar{t}$ and the single-top-quark samples. The $h_{\text {damp }}$ parameter, which controls the $p_{\mathrm{T}}$ of the first additional emission beyond the Born configuration, was set to the mass of the top quark. The main effect of this is to regulate the high- $p_{\mathrm{T}}$ emission against which the $t \bar{t}$ system recoils. The $t \bar{t}$ events are normalized using the cross sections computed at next-to-next-to-leading order (NNLO) with next-to-next-to-leading-logarithmic (NNLL) corrections [57]. The single top quark events are normalized using the NLO + NNLL cross sections for the $W t$-channel [58] and to the NLO cross sections for the $t$ - and $s$-channels [59]. 
TABLE I. Simulated signal and background event samples: the corresponding event generator, parton shower, cross section normalization, PDF set and underlying-event tune are shown.

\begin{tabular}{|c|c|c|c|c|c|}
\hline $\begin{array}{l}\text { Physics process } \\
\text { Signal }\end{array}$ & $\begin{array}{l}\text { Generator } \\
\text { MG5_AMC@NLO 2.2.2 }\end{array}$ & $\begin{array}{l}\text { Parton shower } \\
\text { PYTHIA } 8.186\end{array}$ & $\begin{array}{l}\text { Cross section } \\
\text { NLO + NLL }\end{array}$ & $\begin{array}{c}\text { PDF set } \\
\text { NNPDF2.3 LO }\end{array}$ & $\begin{array}{c}\text { Tune } \\
\text { ATLAS A14 }\end{array}$ \\
\hline$t \bar{t}$ & POWHEG-Box v2 & PYTHIA 6.428 & $\mathrm{NNLO}+\mathrm{NNLL}$ & CT10 NLO & PERUGIA2012 \\
\hline \multicolumn{6}{|l|}{ Single-top } \\
\hline$t$-channel & POWHEG-Box v1 & РYTHIA 6.428 & NLO & CT10f4 NLO & PERUGIA2012 \\
\hline$s$-channel & POWHEG-Box v2 & PYTHIA 6.428 & NLO & CT10 NLO & PERUGIA2012 \\
\hline$W t$-channel & POWHEG-Box v2 & PYTHIA 6.428 & $\mathrm{NLO}+\mathrm{NNLL}$ & CT10 NLO & PERUGIA2012 \\
\hline$W(\rightarrow \ell \nu)+$ jets & SHERPA 2.2 .1 & SHERPA & NNLO & NNPDF3.0 NNLO & SHERPA default \\
\hline$Z / \gamma^{*}(\rightarrow \ell \ell)+$ jets & SHERPA 2.2 .1 & SHERPA & NNLO & NNPDF3.0 NNLO & SHERPA default \\
\hline$W W, W Z$ and $Z Z$ & SHERPA 2.1.1 / SHERPA 2.2.1 & SHERPA & NLO & $\begin{array}{c}\text { CT10 NLO / } \\
\text { NNPDF3.0 NNLO }\end{array}$ & SHERPA default \\
\hline$t \bar{t}+W / Z / W W$ & MG5_AMC@NLO 2.2.2 & PYTHIA 8.186 & NLO & NNPDF2.3 LO & ATLAS A14 \\
\hline
\end{tabular}

Events containing $W$ or $Z$ bosons with associated jets $(W / Z+$ jets) [60] were simulated using the SHERPA 2.2.1 event generator [61]. Matrix elements were calculated for up to two partons at NLO and four partons at LO using the Comix [62] and OpenLoops [63] generators. They were merged with the SHERPA 2.2.1 parton shower [64] with massive $b$ - and $c$-quarks using the ME+PS@NLO prescription [65]. The NNPDF3.0 NNLO PDF set [66] was used in conjunction with a dedicated parton shower tuning developed by the SHERPA authors. The $W / Z+$ jets events are normalized using their NNLO cross sections [67].

The diboson samples [68] were generated using the SHERPA 2.1.1 and 2.2.1 event generators using the CT10 and NNPDF3.0 PDF sets, respectively. The fully leptonic diboson processes were simulated including final states with four charged leptons, three charged leptons and one neutrino, two charged leptons and two neutrinos, and one charged lepton and three neutrinos. The semileptonic diboson processes were simulated with one of the bosons decaying hadronically and the other leptonically. The processes were calculated for up to one parton (for $Z Z$ ) or no additional partons (for $W W, W Z$ ) at NLO and up to three partons at LO.

For the $t \bar{t}+W / Z / W W$ processes [69], all events were simulated using MG5_AMC@NLO 2.2.2 at LO interfaced to the PyTHIA 8.186 parton shower model, with up to two $(t \bar{t}+W)$, one $(t \bar{t}+Z)$ or no $(t \bar{t}+W W)$ extra partons included in the matrix element. The EvtGen 1.2.0 program [43] was used to describe the properties of the bottom and charm hadron decays. The ATLAS shower and underlyingevent tune A14 was used together with the NNPDF2.3 LO PDF set. The events are normalized using their NLO cross sections $[70,71]$.

The response of the detector to particles was modeled either with a full ATLAS detector simulation [72] using GEANT4 [73] or with a fast simulation [74]. The fast simulation is based on a parametrization of the performance of the electromagnetic and hadronic calorimeters and on GEANT4 elsewhere. All background (signal) samples were prepared using the full (fast) detector simulation. All simulated events were generated with a varying number of minimum-bias interactions overlaid on the hardscattering event to model the multiple proton-proton interactions in the same and nearby bunch crossings. The minimum-bias interactions were simulated with the soft QCD processes of PYTHIA 8.186 using the A2 tune [75] and the MSTW2008LO PDF set [76]. Corrections were applied to the samples to account for differences between data and simulation for trigger, identification and reconstruction efficiencies.

The proton-proton data analyzed in this paper were collected by ATLAS during 2015 and 2016 at a centerof-mass energy of $13 \mathrm{TeV}$ with up to 50 simultaneous interactions per proton bunch crossing. After application of data-quality requirements related to the beam and detector conditions, the total integrated luminosity corresponds to $36.1 \mathrm{fb}^{-1}$. The uncertainty in the combined 2015 and 2016 integrated luminosity is $3.2 \%$. It is derived from a calibration of the luminosity scale using $x-y$ beam-separation scans. This methodology is further detailed in Ref. [77].

The data were collected using the higher-level triggers that select events based on the magnitude of the missing transverse momentum, $E_{\mathrm{T}}^{\mathrm{miss}}$. The triggers used are close to fully efficient for events with an offline-reconstructed $E_{\mathrm{T}}^{\text {miss }}$ greater than $200 \mathrm{GeV}$.

\section{EVENT RECONSTRUCTION}

In each event, proton-proton interaction vertices are reconstructed from at least two tracks, each with a transverse momentum $p_{\mathrm{T}}>400 \mathrm{MeV}$ and consistent with the beamspot envelope. The primary vertex (PV) of the event is selected as the vertex with the largest $\sum p_{\mathrm{T}}^{2}$ of the associated tracks.

A distinction is made between preselected and signal leptons and jets. Preselected leptons and jets are used in the $E_{\mathrm{T}}^{\text {miss }}$ computation and are subject to a series of basic 
quality requirements. Signal leptons and jets are a subset of the preselected objects with more stringent requirements and are used for the definition of signal, control and validation regions.

Three-dimensional topological energy clusters in the calorimeters are used as input to the anti- $k_{t}$ algorithm with a radius parameter $R=0.4$ [78-80] to reconstruct preselected jets. The effect of multiple interactions per proton bunch crossing (pileup) is accounted for using the jet area method [81,82]. Subsequent calibrations are applied to the reconstructed jet to improve the energy resolution [83,84]. The residual contamination by pileup jets is further suppressed using a multivariate discriminant that estimates the compatibility of the jet with the PV, as detailed in Ref. [85].

Signal jets must satisfy $p_{\mathrm{T}}>30 \mathrm{GeV}$ and $|\eta|<2.8$. Signal jets within $|\eta|<2.5$ are identified as candidates for containing $b$-hadrons ( $b$-tagged) using the $M V 2 c 10$ algorithm $[86,87]$. This $b$-tagging algorithm provides an overall efficiency of $77 \%$ for jets containing $b$-hadrons in simulated $t \bar{t}$ events, with rejection factors of 6 and 134 on charm and light-jets, respectively [88].

Electron candidates are reconstructed by matching an isolated energy cluster in the electromagnetic calorimeter to at least one ID track. Preselected electrons are identified with the likelihood-based Loose criterion described in Ref. [89] with additional requirements on the number of hits in the innermost pixel layer to discriminate against photon conversions. Furthermore, preselected electrons are required to satisfy $p_{\mathrm{T}}>7 \mathrm{GeV}$ and $|\eta|<2.47$. Muon candidates are formed by a combined refitting of tracks reconstructed in the ID and the MS subsystems. Preselected muons are required to have $p_{\mathrm{T}}>6 \mathrm{GeV}$ and $|\eta|<2.5$, and satisfy the Medium identification criteria in Ref. [90].

To avoid double-counting of the preselected jets, electrons, and muons, a sequence of overlap-removal procedures based on the angular distance $\Delta R=\sqrt{(\Delta y)^{2}+(\Delta \phi)^{2}}$ is applied. First, any jet reconstructed within $\Delta R<0.2$ of a preselected electron is rejected. This prevents electromagnetic energy clusters simultaneously reconstructed as an electron and a jet from being selected twice. Next, to remove bremsstrahlung from muons followed by a photon conversion into electron pairs, electrons within $\Delta R<0.01$ from a preselected muon are discarded. Subsequently, the contamination from muons from decays of heavy hadrons is suppressed by removing muons that are within $\Delta R<\min \left(0.04+(10 \mathrm{GeV}) / p_{\mathrm{T}}^{\mu}, 0.4\right)$ from preselected jets meeting the previous criteria, or $\Delta R<0.2$ from a $b$-tagged jet or a jet containing more than three tracks with $p_{\mathrm{T}}>500 \mathrm{MeV}$. In the former case, the $p_{\mathrm{T}}$-dependent angular separation mitigates the rejection of energetic muons close to jets in boosted event topologies. Finally, jets reconstructed with $\Delta R<0.2$ from a preselected muon are rejected.

Signal electrons are required to satisfy the likelihoodbased tight identification criteria detailed in Ref. [89].
Signal muons and electrons satisfy a sequence of $\eta$ - and $p_{\mathrm{T}}$-dependent isolation requirements on tracking-based and calorimeter-based variables, defined as the GradientLoose [90] isolation criteria. Compatibility of the signal lepton tracks with the PV is enforced by requiring the distance $\left|z_{0} \sin \theta\right|$ to be less than $0.5 \mathrm{~mm}$, where $z_{0}$ is the longitudinal impact parameter. In addition, the transverse impact parameter, $d_{0}$, divided by its uncertainty, $\sigma\left(d_{0}\right)$, must satisfy $\left|d_{0} / \sigma\left(d_{0}\right)\right|<3$ for signal muons and $\left|d_{0} / \sigma\left(d_{0}\right)\right|<5$ for signal electrons.

Corrections derived from data control samples are applied to simulated events to calibrate the reconstruction and identification efficiencies, the momentum scale and resolution of leptons and the efficiency and mistag rate of $b$-tagged jets.

\section{EVENT SELECTION}

Each event must satisfy the trigger selection criteria, and must contain a reconstructed primary vertex. Noncollision background and detector noise are suppressed by rejecting events with any preselected jet not satisfying a set of quality criteria [91]. Exactly one signal lepton, either an electron or a muon, is required. Events with additional preselected leptons are rejected to suppress the dilepton $t \bar{t}$, single-top (Wt-channel), $Z+$ jets and diboson backgrounds. The following observables are used in the definition of signal regions in the analysis.

The missing transverse momentum, $E_{\mathrm{T}}^{\text {miss }}$, is defined as the magnitude of $\boldsymbol{p}_{\mathrm{T}}^{\text {miss }}$, the negative vectorial sum of the transverse momenta of preselected muons, electrons, jets, and identified and calibrated photons. The calculation of $\boldsymbol{p}_{\mathrm{T}}^{\mathrm{miss}}$ also includes the transverse momenta of all tracks originating from the PV and not associated with any identified object $[92,93]$.

The transverse mass, $m_{\mathrm{T}}$, is defined from the lepton transverse momentum $\boldsymbol{p}_{\mathrm{T}}^{\ell}$ and $\boldsymbol{p}_{\mathrm{T}}^{\text {miss }}$ as

$$
m_{\mathrm{T}}=\sqrt{2 p_{\mathrm{T}}^{\ell} E_{\mathrm{T}}^{\mathrm{miss}}\left(1-\cos \left[\Delta \phi\left(\boldsymbol{p}_{\mathrm{T}}^{\ell}, \boldsymbol{p}_{\mathrm{T}}^{\mathrm{miss}}\right)\right]\right)},
$$

where $\Delta \phi\left(\boldsymbol{p}_{\mathrm{T}}^{\ell}, \boldsymbol{p}_{\mathrm{T}}^{\text {miss }}\right)$ is the azimuthal angle between $\boldsymbol{p}_{\mathrm{T}}^{\ell}$ and $\boldsymbol{p}_{\mathrm{T}}^{\text {miss }}$. For $W+$ jets and semileptonic $t \bar{t}$ events, in which one on-shell $W$ boson decays leptonically, the observable has an upper endpoint at the $W$-boson mass. The $m_{\mathrm{T}}$ distribution for signal events extends significantly beyond the distributions of the $W+$ jets and semileptonic $t \bar{t}$ events.

The effective mass, $m_{\text {eff }}$, is the scalar sum of the $p_{\mathrm{T}}$ of the signal lepton and all signal jets and $E_{\mathrm{T}}^{\text {miss: }}$

$$
m_{\mathrm{eff}}=p_{\mathrm{T}}^{\ell}+\sum_{j=1}^{N_{\mathrm{jet}}} p_{\mathrm{T}, j}+E_{\mathrm{T}}^{\mathrm{miss}} .
$$

The effective mass provides good discrimination against SM backgrounds, especially for the signal scenarios where 
energetic jets are expected. Gluino production leads to higher jet multiplicity than squark production. High-mass sparticles tend to produce harder jets than low-mass sparticles. Thus the optimal $m_{\text {eff }}$ value depends on the different signal scenarios. To achieve sensitivity to a wide range of SUSY scenarios with a limited number of signal regions, this variable is binned in the final region definition instead of one simple $m_{\text {eff }}$ cut. The detailed description can be found in Sec. VA.

The transverse momentum scalar sum, $H_{\mathrm{T}}$, is defined as

$$
H_{\mathrm{T}}=\sum_{j=1}^{N_{\mathrm{jet}}} p_{\mathrm{T}, j},
$$

where the index $j$ runs over all the signal jets in the event. Empirically, the experimental resolution of $E_{\mathrm{T}}^{\mathrm{miss}}$ scales with $\sqrt{H_{\mathrm{T}}}$, and the ratio $E_{\mathrm{T}}^{\text {miss }} / \sqrt{H_{\mathrm{T}}}$ is useful for suppressing background events with large $E_{\mathrm{T}}^{\text {miss }}$ due to jet mismeasurement.

The aplanarity is a variable designed to provide more global information about the full momentum tensor of the event. It is defined as $(3 / 2) \times \lambda_{3}$, where $\lambda_{3}$ is the smallest eigenvalue of the normalized momentum tensor [94] calculated using the momenta of the jets and leptons in the event. Typical measured aplanarity values lie in the range $0-0.3$, with values near zero indicating relatively planar backgroundlike events. Signal events tend to have high aplanarity values, since they are more spherical than background events due to multiple objects emitted in the sparticles decay chains.

\section{A. Signal region definitions}

Five sets of event selection criteria, each defining a signal region (SR), are designed to maximize the signal sensitivity. Each SR is labeled by the minimum required number of jets and, optionally, the characteristics of the targeted supersymmetric mass spectrum. Four of the five SRs, 2J, 4J high-x, 4J low-x, and 6J, target the gluino/ squark one-step models. The fifth SR, 9J, targets the gluino two-step and pMSSM models.

Table II summarizes the four SRs targeting the gluino/ squark one-step models. The four SRs are mutually exclusive. For setting model-dependent exclusion limits ("excl"), each of the four SRs is further binned in $b$-veto/ $b$-tag and $m_{\text {eff }}$, and a simultaneous fit is performed across all 28 bins of the four SRs. This choice enhances the sensitivity to a range of new-physics scenarios with different properties such as the presence or absence in the final state of jets containing $b$-hadrons, and different mass separations between the supersymmetric particles. For model-independent limits and null-hypothesis tests ("disc" for discovery), the event yield above a minimum value of $m_{\text {eff }}$ in each SR is used to search for an excess over the SM background.

The $2 \mathrm{~J}$ SR provides sensitivity to scenarios characterized by a relatively heavy $\tilde{\chi}_{1}^{0}$ and small differences between $m_{\tilde{g}}$, $m_{\tilde{\chi}_{1}^{ \pm}}$, and $m_{\tilde{\chi}_{1}^{0}}$, where most of the decay products tend to have small $p_{\mathrm{T}}$. Events with one low- $p_{\mathrm{T}}$ lepton and at least two jets are selected. The minimum lepton $p_{\mathrm{T}}^{\ell}$ is $7(6) \mathrm{GeV}$ for the electron (muon), and the maximum $p_{\mathrm{T}}$ is scaled with the number of signal jets in the event as $5 \mathrm{GeV} \times N_{\text {jet }}$ up to $35 \mathrm{GeV}$. The maximum $p_{\mathrm{T}}^{\ell}$ requirement balances background rejection and signal acceptance for models with increasing mass splittings, where there are more energetic leptons and jets. Stringent requirements on $E_{\mathrm{T}}^{\text {miss }}$ and on $m_{\text {eff }}$ enhance the signal sensitivity by selecting signal events in which the final-state neutralinos are boosted against energetic initial-state radiation (ISR) jets. The SM background is further suppressed by a tight requirement on $E_{\mathrm{T}}^{\mathrm{miss}} / m_{\mathrm{eff}}$.

The $4 \mathrm{~J}$ high-x SR is optimized for models where $m_{\tilde{\chi}_{1}^{0}}$ is fixed to $60 \mathrm{GeV}$ and $x \approx 1$, i.e., $m_{\tilde{\chi}_{1}^{ \pm}}$is close to $m_{\tilde{g}}$. The $W$ boson produced in the chargino decay is significantly

TABLE II. Overview of the selection criteria for the signal regions used for gluino/squark one-step models.

\begin{tabular}{|c|c|c|c|c|}
\hline SR & $2 \mathrm{~J}$ & 4J high- $\mathrm{X}$ & 4J low-X & $6 \mathrm{~J}$ \\
\hline$N_{\ell}$ & $=1$ & $=1$ & $=1$ & $=1$ \\
\hline$p_{\mathrm{T}}^{\ell}[\mathrm{GeV}]$ & $\begin{array}{l}>7(6) \text { for } e(\mu) \text { and } \\
\quad<\min \left(5 \cdot N_{\text {jet }}, 35\right)\end{array}$ & $>35$ & $>35$ & $>35$ \\
\hline$N_{\text {jet }}$ & $\geq 2$ & $4-5$ & $4-5$ & $\geq 6$ \\
\hline$E_{\mathrm{T}}^{\text {miss }}[\mathrm{GeV}]$ & $>430$ & $>300$ & $>250$ & $>350$ \\
\hline$m_{\mathrm{T}}[\mathrm{GeV}]$ & $>100$ & $>450$ & $150-450$ & $>175$ \\
\hline Aplanarity & $\ldots$ & $>0.01$ & $>0.05$ & $>0.06$ \\
\hline$E_{\mathrm{T}}^{\mathrm{miss}} / m_{\mathrm{eff}}$ & $>0.25$ & $>0.25$ & $\cdots$ & $\cdots$ \\
\hline$N_{b \text {-jet }}(\mathrm{excl})$ & \multicolumn{4}{|c|}{$=0$ for $b$-veto, $\geq 1$ for $b$-tag } \\
\hline$m_{\mathrm{eff}}[\mathrm{GeV}](\mathrm{excl})$ & 3 bins & 2 bins & 2 bins & 3 bins \\
\hline$m_{\mathrm{eff}}[\mathrm{GeV}](\mathrm{disc})$ & $\begin{array}{c}\in[700,1900]+[>1900] \\
>1100\end{array}$ & $\begin{array}{c}\in[1000,2000]+[>2000] \\
>1500\end{array}$ & $\begin{array}{c}\in[1300,2000]+[>2000] \\
>1650(1300) \text { for } \\
\text { gluino (squark) }\end{array}$ & $\begin{array}{c}\in[700,2300]+[>2300] \\
>2300(1233) \text { for } \\
\text { gluino (squark) }\end{array}$ \\
\hline
\end{tabular}


TABLE III. Overview of the selection criteria for the signal region used for pMSSM and gluino two-step models.

\begin{tabular}{lc}
\hline \hline SR & $9 \mathrm{~J}$ \\
\hline$N_{\ell}$ & $=1$ \\
$p_{\mathrm{T}}^{\ell}[\mathrm{GeV}]$ & $>35$ \\
$N_{\text {jet }}$ & $\geq 9$ \\
$E_{\mathrm{T}}^{\text {miss }}[\mathrm{GeV}]$ & $>200$ \\
$m_{\mathrm{T}}[\mathrm{GeV}]$ & $>175$ \\
Aplanarity & $>0.07$ \\
$E_{\mathrm{T}}^{\text {miss }} / \sqrt{H_{\mathrm{T}}}\left[\mathrm{GeV}^{1 / 2}\right]$ & $\geq 8$ \\
$m_{\mathrm{eff}}[\mathrm{GeV}](\mathrm{excl})$ & $>1000,1500],[>1500]$ \\
$m_{\mathrm{eff}}[\mathrm{GeV}]($ disc $)$ & $>1500$ \\
\hline \hline
\end{tabular}

boosted, giving rise to a high- $p_{\mathrm{T}}$ lepton. The main characteristics of signal events in this model are large $m_{\mathrm{T}}$ values and relatively soft jets emitted from the sparticle decay. Tight requirements are placed on $E_{\mathrm{T}}^{\text {miss }}, m_{\mathrm{T}}$, and $E_{\mathrm{T}}^{\text {miss }} / m_{\text {eff }}$.

The 4J low-x SR targets models where $m_{\tilde{\chi}_{1}^{0}}$ is fixed to $60 \mathrm{GeV}$ and $x \approx 0$, i.e., $m_{\tilde{\chi}_{1}^{ \pm}}$is close to $m_{\tilde{\chi}_{1}^{0}}$. The large $m_{\tilde{g} / \tilde{q}}-m_{\tilde{\chi}_{1}^{ \pm}}$mass splitting leads to high jet activity, where events are expected to have higher $m_{\text {eff }}$ and larger aplanarity than in the high- $x$ scenarios. The $W$ boson tends to be off-shell, leading to small $m_{\mathrm{T}}$, and accordingly an upper bound is imposed to keep this region orthogonal to the $4 \mathrm{~J}$ high-x SR.

The $6 \mathrm{~J}$ SR is optimized for models with $x=1 / 2$, targeting scenarios with large sparticle mass. Events with one high- $p_{\mathrm{T}}$ lepton and six or more jets are selected. Requirements on $m_{\mathrm{T}}, E_{\mathrm{T}}^{\text {miss }}, m_{\text {eff }}$, and aplanarity are imposed to reduce the SM background from $t \bar{t}$ and $W+$ jets production. The sensitivity is improved for scenarios with large $m_{\tilde{g} / \tilde{q}}$ and small $m_{\tilde{\chi}_{1}^{0}}$ by introducing a higher $m_{\text {eff }}$ bin.

Finally, one signal region, 9J SR, is defined to target the pMSSM and gluino two-step models. The selection criteria are summarized in Table III. At least nine jets are required, targeting the models' long decay chains in which multiple vector or Higgs bosons are produced. The background is further suppressed by tight requirements on the aplanarity and on $E_{\mathrm{T}}^{\text {miss }} / \sqrt{H_{\mathrm{T}}}$. For setting model-dependent exclusion limits ("excl"), the SR is separated into $1000<$ $m_{\text {eff }}<1500 \mathrm{GeV}$ and $m_{\text {eff }}>1500 \mathrm{GeV}$ to achieve good discrimination power for different gluino masses. For model-independent null-hypothesis tests ("disc"), events selected with $m_{\text {eff }}>1500 \mathrm{GeV}$ are used to search for an excess over the SM background.

\section{BACKGROUND ESTIMATION}

The dominant SM backgrounds in most signal regions originate from top quark ( $t \bar{t}$ and single top) and $W+$ jets production. In this section, the techniques employed to estimate the contribution of these backgrounds in the signal regions are detailed.

Additional sources of background in all signal regions originate from the production of $Z+$ jets, $t \bar{t}$ in association with a $W$ or $Z$ boson, and diboson ( $W W, W Z, Z Z)$ events. Their contributions are estimated entirely using simulated event samples normalized to NLO cross sections.

The contribution from multijet processes with a misidentified lepton is found to be negligible once the lepton isolation and $E_{\mathrm{T}}^{\text {miss }}$ requirements used in this search are imposed. A data-driven matrix method, following the implementation described in Ref. [21], determined this in previous iterations of the analysis [22]. As this background is found to be negligible, it is not further considered in the analysis.

The dominant top quark and $W+$ jets backgrounds in the $2 \mathrm{~J}, 4 \mathrm{~J}$ high- $\mathrm{x}, 4 \mathrm{~J}$ low- $\mathrm{x}$, and $6 \mathrm{~J}$ signal regions are estimated by simultaneously normalizing the predicted event yields from simulation to the number of data events observed in dedicated control regions (CR) using the fitting procedure described in Sec. VIII. The simulation is then

TABLE IV. Overview of the control and validation region selection criteria corresponding to the $2 \mathrm{~J} \mathrm{SR}$. The top and $W+$ jets control regions are denoted by TR and WR, respectively.

\begin{tabular}{|c|c|c|c|}
\hline $2 \mathrm{~J}$ & WR/TR & VR $E_{\mathrm{T}}^{\text {miss }}$ & $\mathrm{VR} m_{\mathrm{T}}$ \\
\hline$N_{\ell}$ & \multicolumn{3}{|c|}{$=1$} \\
\hline$p_{\mathrm{T}}^{e}[\mathrm{GeV}]$ & \multicolumn{3}{|c|}{$>7(6)$ for $e(\mu)$ and $<\min \left(5 \cdot N_{\mathrm{jet}}, 35\right)$} \\
\hline$N_{\text {jet }}$ & \multicolumn{3}{|c|}{$\geq 2$} \\
\hline$N_{b \text {-jet }}$ & $=0 / \geq 1$ & $\cdots$ & $\cdots$ \\
\hline$m_{\mathrm{T}}[\mathrm{GeV}]$ & {$[40,100]$} & {$[40,100]$} & $>100$ \\
\hline$E_{\mathrm{T}}^{\text {miss }}[\mathrm{GeV}]$ & {$[300,430]$} & $>430$ & {$[300,430]$} \\
\hline Aplanarity & $\ldots$ & $\ldots$ & $\ldots$ \\
\hline$E_{\mathrm{T}}^{\mathrm{miss}} / m_{\mathrm{eff}}$ & $>0.15$ & $>0.25$ & $>0.1$ \\
\hline$m_{\text {eff }}[\mathrm{GeV}]$ & \multicolumn{3}{|c|}{3 bins $\in[700,1900]+[>1900]$} \\
\hline
\end{tabular}

TABLE V. Overview of the control and validation region selection criteria corresponding to the $4 \mathrm{~J}$ high-x SR. The top and $W+$ jets control regions are denoted by TR and WR, respectively.

\begin{tabular}{lcrcc}
\hline \hline 4J high-x & WR/TR & VR Aplanarity & VR $m_{\mathrm{T}}$ & VR Hybrid \\
\hline$N_{\ell}$ & \multicolumn{5}{c}{$=1$} \\
$p_{\mathrm{T}}^{\ell}[\mathrm{GeV}]$ & \multicolumn{5}{c}{$>35$} \\
$N_{\text {jet }}$ & $=0 / \geq 1$ & $\ldots$ & $\ldots$ & $\ldots$ \\
$N_{b \text {-jet }}$ & $\ldots, 5$ & & \\
$m_{\mathrm{T}}[\mathrm{GeV}]$ & {$[50,200]$} & {$[50,150]$} & $>200$ & {$[150,450]$} \\
$E_{\mathrm{T}}^{\text {miss }}[\mathrm{GeV}]$ & $>300$ & $>250$ & $>250$ & $>250$ \\
Aplanarity & $<0.01$ & $>0.05$ & $<0.01$ & {$[0.01,0.05]$} \\
$E_{\mathrm{T}}^{\text {miss }} / m_{\mathrm{eff}}$ & $>0.25$ & $>0.25$ & $>0.25$ & $\cdots$ \\
$m_{\mathrm{eff}}[\mathrm{GeV}]$ & \multicolumn{5}{c}{2 bins $\in[1000,2000]+[>2000]$} \\
\hline \hline
\end{tabular}


TABLE VI. Overview of the control and validation region selection criteria corresponding to the $4 \mathrm{~J}$ low-x SR. The top and $W+$ jets control regions are denoted by TR and WR, respectively.

\begin{tabular}{lccc}
\hline \hline $4 \mathrm{~J}$ low-x & WR/TR & VR Aplanarity & VR Hybrid \\
\hline$N_{\ell}$ & & $=1$ & \\
$p_{\mathrm{T}}^{\ell}[\mathrm{GeV}]$ & & $>35$ & \\
$N_{\text {jet }}$ & $=0 / \geq 1$ & $\cdots$ & $\cdots$ \\
$N_{b \text {-jet }}$ & {$[50,150]$} & {$[50,150]$} & {$[150,450]$} \\
$m_{\mathrm{T}}[\mathrm{GeV}]$ & & $>250$ & \\
$E_{\mathrm{T}}^{\text {miss }}[\mathrm{GeV}]$ & {$[0.01,0.05]$} & $>0.05$ & {$[0.01,0.05]$} \\
Aplanarity & 2 bins $\in[1300,2000]+[>2000]$ \\
$m_{\text {eff }}[\mathrm{GeV}]$ & \multicolumn{3}{l}{} \\
\hline \hline
\end{tabular}

TABLE VII. Overview of the control and validation region selection criteria corresponding to the $6 \mathrm{~J}$ SR. The top and $W+$ jets control regions are denoted by TR and WR, respectively.

\begin{tabular}{lccc}
\hline \hline $6 \mathrm{~J}$ & $\mathrm{WR} / \mathrm{TR}$ & VR Aplanarity & $\mathrm{VR} m_{\mathrm{T}}$ \\
\hline$N_{\ell}$ & & $=1$ & \\
$p_{\mathrm{T}}^{\ell}[\mathrm{GeV}]$ & & $>35$ & \\
$N_{\text {jet }}$ & $=0 / \geq 1$ & $\cdots$ & $\ldots$ \\
$N_{b \text {-jet }}$ & {$[50,175]$} & {$[50,175]$} & {$[175,400]$} \\
$m_{\mathrm{T}}[\mathrm{GeV}]$ & $>350$ & $>350$ & $>250$ \\
$E_{\mathrm{T}}^{\text {miss }}[\mathrm{GeV}]$ & $<0.06$ & $>0.06$ & $<0.06$ \\
Aplanarity & \multicolumn{3}{c}{3 bins $\in[700,2300]+[>2300]$} \\
$m_{\text {eff }}[\mathrm{GeV}]$ & \multicolumn{3}{c}{} \\
\hline \hline
\end{tabular}

used to extrapolate the measured background rates to the corresponding signal regions.

The CRs are designed to have high purity in the background process of interest, a sufficiently large number of events to obtain small statistical uncertainties in the background prediction, and a small contamination by events from the signal models under consideration. Moreover, they are designed to have kinematic properties resembling as closely as possible those of the signal regions, in order to provide good estimates of the kinematics of background processes there. This procedure limits the impact of potentially large systematic uncertainties in the expected yields from the extrapolation.

Tables IV-VII list the criteria that define the control regions corresponding to signal regions $2 \mathrm{~J}, 4 \mathrm{~J}$ high- $\mathrm{x}, 4 \mathrm{~J}$ low-X, and 6J. As described in Sec. V, these signal regions contain multiple bins in $m_{\text {eff }}$. The same binning is maintained for the control regions, so that every signal region bin in $m_{\text {eff }}$ has corresponding control regions with the same requirements on $m_{\text {eff }}$ and, therefore, the backgrounds are estimated independently in each $m_{\mathrm{eff}}$ bin.

Dedicated top and $W+$ jets control regions, respectively denoted by TR and WR, are constructed in each bin of $m_{\text {eff }}$. The TR and WR are distinguished by requiring at least one or exactly zero $b$-tagged signal jets, respectively. Cross-contamination from top and $W+$ jets processes between these two types of control regions is accounted for in the fit. The measured top and $W+$ jets background rates from the TR and WR regions in a given $m_{\text {eff }}$ bin are extrapolated to the signal region within the same $m_{\text {eff }}$ bin. The signal regions in a given $m_{\text {eff }}$ bin may be further separated into regions with at least one or exactly zero $b$-tagged signal jets as described in Sec. V. For such signal regions separated by $b$-tagged jet multiplicity, the extrapolation is performed from both the TR and WR regions to each individual bin of $b$-tagged jet multiplicity.

To validate the extrapolation from control to signal regions using simulated event samples, dedicated validation regions (VRs) are defined for each set of control and signal regions. The selection criteria defining these VRs are also shown in Tables IV-VII. The same binning in $m_{\mathrm{eff}}$ used in the control and signal regions is also maintained in the validation regions. The VRs are designed to be kinematically close to the signal regions, with only a small contamination from the signal in the models considered in this search. The VRs are not used to constrain parameters in

TABLE VIII. Overview of the control and validation region selection criteria corresponding to the 9J SR. The control regions $\mathrm{CR}_{\mathrm{A}, \mathrm{A}^{\prime} \mathrm{B}, \mathrm{C}, \mathrm{C}^{\prime}}$ are further divided into bins of exactly 0 or $\geq 1 b$-tagged signal jets to enrich top and $W+$ jets backgrounds, respectively.

\begin{tabular}{|c|c|c|c|c|c|c|c|}
\hline $9 \mathrm{~J}$ & $\mathrm{CR}_{\mathrm{A}}$ & $\mathrm{CR}_{\mathrm{B}}$ & $\mathrm{CR}_{\mathrm{C}}$ & $\mathrm{VR} m_{\mathrm{T}}$ & $\mathrm{CR}_{\mathrm{C}^{\prime}}$ & $\mathrm{VR} N_{\text {jet }}$ & $\mathrm{CR}_{\mathrm{A}^{\prime}}$ \\
\hline $\begin{array}{l}N_{\ell} \\
p_{\mathrm{T}}^{\ell}[\mathrm{GeV}]\end{array}$ & & & & $\begin{array}{c}=1 \\
\geq 35\end{array}$ & & & \\
\hline$N_{\text {jet }}$ & $5-6$ & $5-6$ & $\geq 9$ & $7-8$ & $7-8$ & $\geq 9$ & $5-6$ \\
\hline $\begin{array}{l}p_{\mathrm{T}}^{\mathrm{jet}}[\mathrm{GeV}] \\
N_{b \text {-jet }}\end{array}$ & $=0 / \geq 1$ & $=0 / \geq 1$ & $=0 / \geq 1$ & $\begin{array}{l}\geq 30 \\
\ldots\end{array}$ & $=0 / \geq 1$ & $\cdots$ & $=0 / \geq 1$ \\
\hline $\begin{array}{l}m_{\mathrm{T}}[\mathrm{GeV}] \\
E_{\mathrm{T}}^{\text {miss }}[\mathrm{GeV}] \\
E_{\mathrm{T}}^{\text {miss }} / \sqrt{H_{\mathrm{T}}}\end{array}$ & $>175$ & $<100$ & $<100$ & $\begin{array}{c}>175 \\
>200 \\
\geq 8\end{array}$ & $<100$ & {$[100,175]$} & {$[100,175]$} \\
\hline $\begin{array}{l}\text { Aplanarity } \\
m_{\text {eff }}[\mathrm{GeV}]\end{array}$ & $\begin{array}{c}\cdots \\
>1000\end{array}$ & $\begin{array}{c}\cdots \\
>1000\end{array}$ & $\begin{array}{l}>0.07 \\
>1000\end{array}$ & $\begin{array}{c}<0.05 \\
{[1000,1500],[>1500]}\end{array}$ & $\begin{array}{c}<0.05 \\
>1000\end{array}$ & $\begin{array}{c}<0.05 \\
>1000\end{array}$ & $\begin{array}{c}\cdots \\
>1000\end{array}$ \\
\hline
\end{tabular}




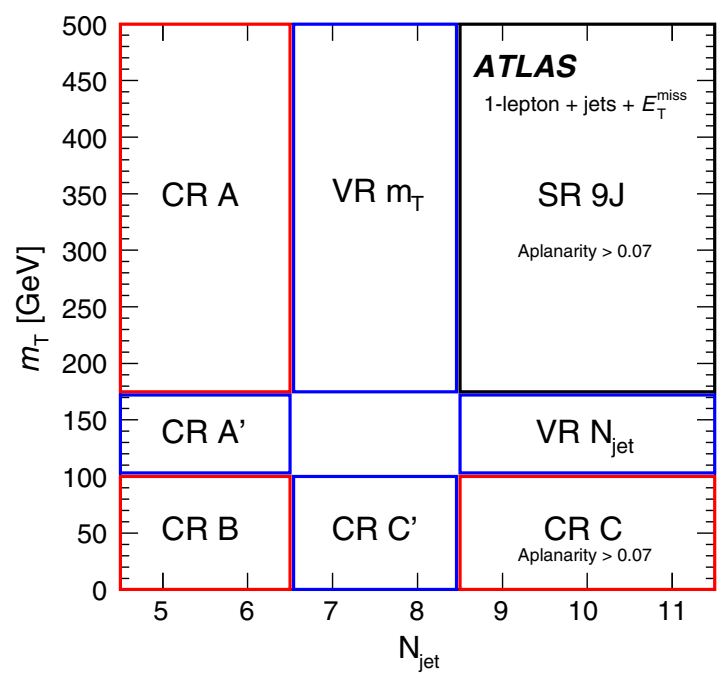

FIG. 2. Illustration of the control and validation region configuration corresponding to the 9J SR. The control regions that are used for the background estimation in the signal region are indicated by red lines, while the blue lines indicate control regions that are used for the background estimation in the validation regions and the validation regions themselves. The definitions for all regions are given in Table III (signal region) and Table VIII (control and validation regions).

the fit, but provide a statistically independent cross-check of the extrapolation. The observed event yields in the VRs are found to be consistent with the background prediction as further discussed in Sec. VIII.
One of the dominant background components in the $2 \mathrm{~J}$, 4J high-x, 4J low-x, and $6 \mathrm{~J}$ SRs is $t \bar{t}$ production with dileptonic final state, where one lepton fails to be reconstructed ("missing lepton") or is a semihadronically decaying $\tau$ lepton; this background is characterized by high values of $m_{\mathrm{T}}$. To validate the above described background estimation technique, which is largely a simulation-based extrapolation from low- $m_{\mathrm{T}}$ control regions populated by events with semileptonic $t \bar{t}$ decays, an alternative method was developed. This method (hereafter referred to as the object replacement method) uses events in a dileptonic control region. To emulate the missing lepton case, the $p_{\mathrm{T}}$ of one of the two leptons is added vectorially to the calculation of $E_{\mathrm{T}}^{\text {miss }}$. To emulate the hadronic $\tau$ decay case, one of the two leptons is resimulated as a hadronic tau decay using the Tauola generator [95] with appropriate energy scale and resolution corrections. The accuracy of this alternative background estimation technique was validated on simulated samples as well as in data validation regions. The background estimates derived from this object replacement method are found to be consistent with those obtained from the standard semi-data-driven approach as further demonstrated in Sec. VIII.

While the background estimation strategy described above works well for the signal regions $2 \mathrm{~J}$, $4 \mathrm{~J}$ high-x, $4 \mathrm{~J}$ low-x, and $6 \mathrm{~J}$, it is not viable for the $9 \mathrm{~J}$ SR. The reason for this is that the simulation-based extrapolation from the control regions, which are typically located around the peak region of the transverse mass distribution $\left(m_{\mathrm{T}} \sim 80 \mathrm{GeV}\right)$,

TABLE IX. Breakdown of the dominant systematic uncertainties in the background estimates in the 2J and 4J high-x SRs. The individual uncertainties can be correlated and do not necessarily add up in quadrature to the total background uncertainty. The percentages show the size of the uncertainty relative to the total expected background.

\begin{tabular}{lcccc}
\hline \hline Signal region & 2J $b$-tag & 2J $b$-veto & 4J high-x $b$-tag & 4J high-x $b$-veto \\
\hline Total background expectation & 47 & 36 & 54 & 44 \\
\hline Total background systematic uncertainty & $\pm 4[9 \%]$ & $\pm 9[24 \%]$ & $\pm 7[12 \%]$ & $\pm 10[23 \%]$ \\
\hline Experimental uncertainty & \pm 1.3 & \pm 2.2 & \pm 2.6 & \pm 5 \\
Normalization uncertainty & \pm 2.8 & \pm 0.9 & \pm 4 & \pm 1.9 \\
Theoretical uncertainty & \pm 3.5 & \pm 9 & \pm 1.7 & \pm 6 \\
Statistical uncertainty of MC samples & \pm 1.4 & \pm 1.8 & \pm 7 \\
\hline \hline
\end{tabular}

TABLE X. Breakdown of the dominant systematic uncertainties in the background estimates in the 4J low-X and 6J SRs. The individual uncertainties can be correlated and do not necessarily add up in quadrature to the total background uncertainty. The percentages show the size of the uncertainty relative to the total expected background.

\begin{tabular}{lcccc}
\hline \hline Signal region & 4J low-x $b$-tag & 4J low-x $b$-veto & $6 \mathrm{~J} b$-tag & $6 \mathrm{~J} b$-veto \\
\hline Total background expectation & 31 & 16 & 27 & 7.3 \\
\hline Total background systematic uncertainty & $\pm 6[21 \%]$ & $\pm 4[25 \%]$ & $\pm 4[15 \%]$ & $\pm 2.0[27 \%]$ \\
\hline Experimental uncertainty & \pm 1.8 & \pm 1.0 & \pm 1.1 & \pm 0.8 \\
Normalization uncertainty & \pm 2.3 & \pm 0.8 & \pm 1.4 & \pm 0.5 \\
Theoretical uncertainty & \pm 6 & \pm 4 & \pm 4 & \pm 2.0 \\
Statistical uncertainty of MC samples & \pm 1.2 & \pm 1.0 & \pm 0.9 & \pm 0.6 \\
\hline \hline
\end{tabular}


TABLE XI. Breakdown of the dominant systematic uncertainties in the background estimates in the 9J SR. The individual uncertainties can be correlated and do not necessarily add up in quadrature to the total background uncertainty. The percentage shows the size of the uncertainty relative to the total expected background.

\begin{tabular}{lc}
\hline \hline Signal region & $9 \mathrm{~J}$ \\
\hline Total background expectation & 7 \\
\hline Total background systematic uncertainty & $\pm 4[50 \%]$ \\
\hline Theoretical uncertainty & \pm 4 \\
Normalization uncertainty & \pm 2.0 \\
Experimental uncertainty & \pm 1.9 \\
Statistical uncertainty of MC samples & \pm 0.7 \\
\hline \hline
\end{tabular}

to the high- $m_{\mathrm{T}}$ signal regions $\left(m_{\mathrm{T}} \gg 80 \mathrm{GeV}\right)$ is affected by large theoretical uncertainties at high jet multiplicities. Because the peak and tail regions of the $m_{\mathrm{T}}$ distribution are dominated by semileptonic and dileptonic final states from $t \bar{t}$ decays, respectively, additional jets from initial- or finalstate radiation are required to obtain the same jet multiplicity for dileptonic $t \bar{t}$ final states. Inadequate modeling of such additional jets is the dominant source of the theoretical uncertainty. To reduce the dependence on the modeling of additional jets, a dedicated data-driven background estimation technique was designed for the 9J SR. The method relies on the assumption that the $m_{\mathrm{T}}$ distribution is approximately invariant under changes in the jet

TABLE XII. Event yields and background-only fit results for the $2 \mathrm{~J}$ and $4 \mathrm{~J}$ high-X SRs. Each column corresponds to a bin in $m_{\text {eff }}$ $[\mathrm{GeV}]$. Uncertainties in the fitted background estimates combine statistical (in the simulated event yields) and systematic uncertainties. The uncertainties in this table are symmetrized for propagation purposes but truncated at zero to remain within the physical boundaries.

\begin{tabular}{|c|c|c|c|c|c|}
\hline $2 \mathrm{~J} b$-tag & All $m_{\text {eff }}$ bins & {$[700,1100]$} & {$[1100,1500]$} & {$[1500,1900]$} & $>1900[\mathrm{GeV}]$ \\
\hline Observed events & 47 & 8 & 21 & 12 & 6 \\
\hline Fitted bkg events & $47 \pm 4$ & $6.0 \pm 1.3$ & $23.0 \pm 3.0$ & $12.9 \pm 2.1$ & $5.2 \pm 1.8$ \\
\hline$\overline{\text { Fitted } t \bar{t} \text { events }}$ & $31.1 \pm 3.5$ & $2.7 \pm 0.8$ & $15.3 \pm 2.5$ & $9.4 \pm 1.7$ & $3.8 \pm 1.4$ \\
\hline Fitted $W+$ jets events & $3.7 \pm 1.4$ & $1.1 \pm 0.9$ & $2.0 \pm 1.0$ & $0.5 \pm 0.4$ & $0.10 \pm 0.10$ \\
\hline Fitted $Z+$ jets events & $2.0 \pm 0.6$ & $0.70 \pm 0.20$ & $0.90 \pm 0.30$ & $0.30 \pm 0.10$ & $0.10 \pm 0.10$ \\
\hline Fitted single-top events & $5.6 \pm 1.8$ & $0.6 \pm 0.4$ & $2.7 \pm 1.3$ & $1.5 \pm 1.0$ & $0.8 \pm 0.8$ \\
\hline Fitted diboson events & $1.8 \pm 1.4$ & $0.30 \pm 0.20$ & $0.8 \pm 0.8$ & $0.50 \pm 0.30$ & $0.10 \pm 0.10$ \\
\hline Fitted $t \bar{t}+\mathrm{V}$ events & $2.92 \pm 0.25$ & $0.60 \pm 0.10$ & $1.30 \pm 0.10$ & $0.70 \pm 0.10$ & $0.30 \pm 0.04$ \\
\hline $2 \mathrm{~J} b$-veto & All $m_{\text {eff }}$ bins & {$[700,1100]$} & {$[1100,1500]$} & {$[1500,1900]$} & $>1900[\mathrm{GeV}]$ \\
\hline Observed events & 61 & 20 & 26 & 9 & 6 \\
\hline Fitted bkg events & $36 \pm 9$ & $10 \pm 4$ & $16 \pm 5$ & $7.0 \pm 1.9$ & $2.5 \pm 0.8$ \\
\hline$\overline{\text { Fitted } t \bar{t} \text { events }}$ & $5.1 \pm 1.0$ & $1.00 \pm 0.30$ & $2.7 \pm 0.6$ & $1.00 \pm 0.30$ & $0.40 \pm 0.20$ \\
\hline Fitted $W+$ jets events & $13 \pm 4$ & $5 \pm 4$ & $5.8 \pm 1.8$ & $2.4 \pm 1.1$ & $0.50 \pm 0.30$ \\
\hline Fitted $Z+$ jets events & $5.8 \pm 1.7$ & $1.8 \pm 0.6$ & $2.3 \pm 0.8$ & $1.00 \pm 0.30$ & $0.60 \pm 0.30$ \\
\hline Fitted single-top events & $1.1 \pm 0.4$ & $0.10 \pm 0.10$ & $0.70 \pm 0.30$ & $0.20 \pm 0.10$ & $0.10 \pm 0.10$ \\
\hline Fitted diboson events & $10 \pm 8$ & $2.5 \pm 1.7$ & $4 \pm 4$ & $2.3 \pm 1.5$ & $0.8 \pm 0.7$ \\
\hline Fitted $t \bar{t}+\mathrm{V}$ events & $0.34 \pm 0.08$ & $0.048 \pm 0.019$ & $0.20 \pm 0.10$ & $0.066 \pm 0.013$ & $0.025 \pm 0.007$ \\
\hline 4J high-x $b$-tag & All $m_{\text {eff }}$ bins & \multicolumn{2}{|c|}{$[1000,1500]$} & {$[1500,2000]$} & $>2000[\mathrm{GeV}]$ \\
\hline Observed events & 44 & \multicolumn{2}{|c|}{38} & 4 & 2 \\
\hline Fitted bkg events & $54 \pm 7$ & \multicolumn{2}{|c|}{$44 \pm 6$} & $7.7 \pm 1.5$ & $1.8 \pm 0.7$ \\
\hline$\overline{\text { Fitted } t \bar{t} \text { events }}$ & $39 \pm 6$ & \multicolumn{2}{|c|}{$34 \pm 6$} & $4.9 \pm 1.2$ & $0.8 \pm 0.5$ \\
\hline Fitted $W+$ jets events & $3.2 \pm 1.5$ & \multicolumn{2}{|c|}{$2.7 \pm 1.5$} & $0.20 \pm 0.10$ & $0.20 \pm 0.10$ \\
\hline Fitted $Z+$ jets events & $0.14 \pm 0.13$ & \multicolumn{2}{|c|}{$0.10 \pm 0.10$} & $0.036 \pm 0.025$ & $0.007_{-0.007}^{+0.019}$ \\
\hline Fitted single-top events & $6.0 \pm 2.3$ & \multicolumn{2}{|c|}{$4.1 \pm 2.2$} & $1.6 \pm 0.7$ & $0.30 \pm 0.30$ \\
\hline Fitted diboson events & $1.6 \pm 1.0$ & \multicolumn{2}{|c|}{$1.0 \pm 0.6$} & $0.30 \pm 0.30$ & $0.30 \pm 0.30$ \\
\hline Fitted $t \bar{t}+\mathrm{V}$ events & $3.69 \pm 0.25$ & \multicolumn{2}{|c|}{$2.84 \pm 0.18$} & $0.70 \pm 0.10$ & $0.155 \pm 0.026$ \\
\hline $4 \mathrm{~J}$ high-x $b$-veto & All $m_{\text {eff }}$ bins & \multicolumn{2}{|c|}{$[1000,1500]$} & {$[1500,2000]$} & $>2000[\mathrm{GeV}]$ \\
\hline Observed events & 37 & \multicolumn{2}{|c|}{27} & 7 & 3 \\
\hline Fitted bkg events & $44 \pm 10$ & \multicolumn{2}{|c|}{$36 \pm 10$} & $5.8 \pm 1.9$ & $2.4 \pm 1.0$ \\
\hline$\overline{\text { Fitted } t \bar{t} \text { events }}$ & $4.0 \pm 1.1$ & \multicolumn{2}{|c|}{$3.3 \pm 1.0$} & $0.60 \pm 0.20$ & $0.10 \pm 0.10$ \\
\hline Fitted $W+$ jets events & $28 \pm 8$ & \multicolumn{2}{|c|}{$24 \pm 8$} & $3.0 \pm 1.1$ & $1.0 \pm 0.4$ \\
\hline Fitted $Z+$ jets events & $1.4 \pm 0.8$ & \multicolumn{2}{|c|}{$0.9 \pm 0.5$} & $0.10 \pm 0.10$ & $0.30 \pm 0.30$ \\
\hline Fitted single-top events & $0.9 \pm 0.4$ & \multicolumn{2}{|c|}{$0.6 \pm 0.4$} & $0.20 \pm 0.10$ & $0.10 \pm 0.10$ \\
\hline Fitted diboson events & $9 \pm 5$ & \multicolumn{2}{|c|}{$6.2 \pm 3.4$} & $1.8 \pm 1.5$ & $0.9 \pm 0.8$ \\
\hline Fitted $t \bar{t}+\mathrm{V}$ events & $0.36 \pm 0.09$ & \multicolumn{2}{|c|}{$0.25 \pm 0.07$} & $0.057 \pm 0.018$ & $0.054 \pm 0.024$ \\
\hline
\end{tabular}


multiplicity requirements. This assumption is found to be valid when tight $m_{\text {eff }}$ requirements as used in this analysis are applied such that the overall activity in the calorimeter and thus the missing transverse momentum resolution are not significantly affected by variations in the jet multiplicity. Based on the $m_{\mathrm{T}}$ invariance, mutually exclusive control regions $\mathrm{CR}_{\mathrm{A}, \mathrm{B}, \mathrm{C}}$ are defined in the $m_{\mathrm{T}}-N_{\text {jet }}$ plane, where $\mathrm{CR}_{\mathrm{A}}$ is located at high $m_{\mathrm{T}}$ and low $N_{\text {jet }}, \mathrm{CR}_{\mathrm{B}}$ at low $m_{\mathrm{T}}$ and low $N_{\text {jet }}$, and $\mathrm{CR}_{\mathrm{C}}$ at low $m_{\mathrm{T}}$ and high $N_{\text {jet }}$. The precise requirements of these regions are defined in Table VIII and illustrated in Fig. 2. Based on these regions, the background in the high $m_{\mathrm{T}}$ and high $N_{\text {jet }}$ signal region can then be estimated with the following equation:

$$
\frac{N_{\mathrm{CR}_{\mathrm{A}}}}{N_{\mathrm{CR}_{\mathrm{B}}}}=\frac{N_{\mathrm{SR}_{9 \mathrm{~J}}}^{\mathrm{est}}}{N_{\mathrm{CR}_{\mathrm{C}}}} \rightarrow N_{\mathrm{SR}_{9 \mathrm{~J}}}^{\mathrm{est}}=\frac{N_{\mathrm{CR}_{\mathrm{A}}}}{N_{\mathrm{CR}_{\mathrm{B}}}} N_{\mathrm{CR}_{\mathrm{C}}},
$$

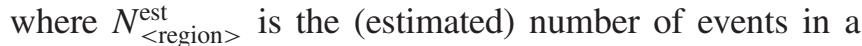
given region. The residual small correlations between $m_{\mathrm{T}}$ and $N_{\text {jet }}$ that bias the background estimate in the signal region can then be expressed in terms of a simulation-based closure parameter defined as

TABLE XIII. Event yields and background-only fit results for the 4J low-x and 6J SRs. Each column corresponds to a bin in $m_{\text {eff }}$ $[\mathrm{GeV}]$. Uncertainties in the fitted background estimates combine statistical (in the simulated event yields) and systematic uncertainties. The uncertainties in this table are symmetrized for propagation purposes but truncated at zero to remain within the physical boundaries.

\begin{tabular}{|c|c|c|c|c|c|}
\hline 4J low-x $b$-tag & \multicolumn{3}{|c|}{$[1300,1650]$} & {$[1650,2000]$} & $>2000[\mathrm{GeV}]$ \\
\hline Observed events & 31 & \multicolumn{2}{|c|}{19} & 6 & 6 \\
\hline Fitted bkg. events & $31 \pm 6$ & \multicolumn{2}{|c|}{$20 \pm 5$} & $6.6 \pm 2.3$ & $4.4 \pm 1.6$ \\
\hline Fitted $t \bar{t}$ events & $19 \pm 5$ & \multicolumn{2}{|c|}{$13 \pm 4$} & $4.3 \pm 1.6$ & $2.1 \pm 1.0$ \\
\hline Fitted $W+$ jets events & $2.4 \pm 1.1$ & \multicolumn{2}{|c|}{$1.6 \pm 1.1$} & $0.40 \pm 0.20$ & $0.40 \pm 0.20$ \\
\hline Fitted $Z+$ jets events & $0.10 \pm 0.05$ & \multicolumn{2}{|c|}{$0.037 \pm 0.033$} & $0.017 \pm 0.014$ & $0.042 \pm 0.030$ \\
\hline Fitted single-top events & $6.5 \pm 3.4$ & \multicolumn{2}{|c|}{$3.5 \pm 2.8$} & $1.5 \pm 1.5$ & $1.5 \pm 1.1$ \\
\hline Fitted diboson events & $1.2 \pm 0.9$ & \multicolumn{2}{|c|}{$0.7 \pm 0.4$} & $0.20 \pm 0.20$ & $0.30 \pm 0.30$ \\
\hline Fitted $t \bar{t}+\mathrm{V}$ events & $1.41 \pm 0.14$ & \multicolumn{2}{|c|}{$1.03 \pm 0.12$} & $0.24 \pm 0.05$ & $0.14 \pm 0.04$ \\
\hline $4 \mathrm{~J}$ low-x $b$-veto & All $m_{\text {eff }}$ bins & \multicolumn{2}{|c|}{$[1300,1650]$} & {$[1650,2000]$} & $>2000[\mathrm{GeV}]$ \\
\hline Observed events & 19 & \multicolumn{2}{|c|}{7} & 7 & 5 \\
\hline Fitted bkg events & $16 \pm 4$ & \multicolumn{2}{|c|}{$10.0 \pm 3.0$} & $3.5 \pm 1.1$ & $2.7 \pm 1.1$ \\
\hline$\overline{\text { Fitted } t \bar{t} \text { events }}$ & $2.3 \pm 0.8$ & \multicolumn{2}{|c|}{$1.6 \pm 0.6$} & $0.40 \pm 0.20$ & $0.30 \pm 0.20$ \\
\hline Fitted $W+$ jets events & $7.5 \pm 2.6$ & \multicolumn{2}{|c|}{$4.6 \pm 2.3$} & $1.9 \pm 0.8$ & $1.0 \pm 0.5$ \\
\hline Fitted $Z+$ jets events & $0.18 \pm 0.09$ & \multicolumn{2}{|c|}{$0.06_{-0.06}^{+0.06}$} & $0.05 \pm 0.04$ & $0.070 \pm 0.032$ \\
\hline Fitted single-top events & $1.2 \pm 0.7$ & \multicolumn{2}{|c|}{$0.5 \pm 0.4$} & $0.20 \pm 0.20$ & $0.5 \pm 0.4$ \\
\hline Fitted diboson events & $4.9 \pm 3.3$ & \multirow{2}{*}{\multicolumn{2}{|c|}{$\begin{aligned} 3.2 & \pm 1.9 \\
0.099 & \pm 0.023\end{aligned}$}} & $1.0 \pm 0.7$ & $0.7_{-0.7}^{+0.9}$ \\
\hline Fitted $t \bar{t}+\mathrm{V}$ events & $0.125 \pm 0.031$ & & & $0.014 \pm 0.008$ & $0.012_{-0.012}^{+0.014}$ \\
\hline $6 \mathrm{~J} b$-tag & All $m_{\text {eff }}$ bins & {$[700,1233]$} & {$[1233,1767]$} & {$[1767,2300]$} & $>2300[\mathrm{GeV}]$ \\
\hline Observed events & 31 & 6 & 16 & 9 & 0 \\
\hline Fitted bkg events & $27 \pm 4$ & $8.5 \pm 2.0$ & $12.1 \pm 2.7$ & $4.1 \pm 1.0$ & $2.0 \pm 0.5$ \\
\hline$\overline{\text { Fitted } t \bar{t} \text { events }}$ & $19 \pm 4$ & $6.1 \pm 1.8$ & $9.1 \pm 2.5$ & $2.6 \pm 0.8$ & $1.1 \pm 0.4$ \\
\hline Fitted $W+$ jets events & $0.75 \pm 0.29$ & $0.20 \pm 0.20$ & $0.30 \pm 0.10$ & $0.20 \pm 0.10$ & $0.10 \pm 0.10$ \\
\hline Fitted $Z+$ jets events & $0.07 \pm 0.06$ & & $0.025_{-0.025}^{+0.026}$ & $0.029_{-0.029}^{+0.035}$ & $0.014 \pm 0.009$ \\
\hline Fitted single-top events & $3.1 \pm 1.1$ & $0.7 \pm 0.5$ & $1.3 \pm 0.8$ & $0.6 \pm 0.4$ & $0.40 \pm 0.30$ \\
\hline Fitted diboson events & $0.8 \pm 0.7$ & $0.20 \pm 0.20$ & $0.30 \pm 0.20$ & $0.20 \pm 0.20$ & $0.10 \pm 0.10$ \\
\hline Fitted $t \bar{t}+\mathrm{V}$ events & $2.9 \pm 0.5$ & $1.20 \pm 0.20$ & $1.10 \pm 0.30$ & $0.50 \pm 0.10$ & $0.2 \pm 0.0$ \\
\hline $6 \mathrm{~J} b$-veto & All $m_{\text {eff }}$ bins & {$[700,1233]$} & {$[1233,1767]$} & {$[1767,2300]$} & $>2300[\mathrm{GeV}]$ \\
\hline Observed events & 6 & 3 & 2 & 1 & 0 \\
\hline Fitted bkg events & $7.3 \pm 2.0$ & $2.1 \pm 1.1$ & $3.4 \pm 0.8$ & $1.2 \pm 0.5$ & $0.60 \pm 0.30$ \\
\hline Fitted $t \bar{t}$ events & $1.6 \pm 0.5$ & $0.50 \pm 0.20$ & $0.80 \pm 0.30$ & $0.20 \pm 0.10$ & $0.069 \pm 0.031$ \\
\hline Fitted $W+$ jets events & $2.4 \pm 0.9$ & $0.6_{-0.6}^{+0.8}$ & $1.1 \pm 0.4$ & $0.50 \pm 0.30$ & $0.20 \pm 0.10$ \\
\hline Fitted $Z+$ jets events & $0.29 \pm 0.13$ & $0.1 \pm 0.0$ & $0.20 \pm 0.10$ & $0.026 \pm 0.015$ & $0.038 \pm 0.032$ \\
\hline Fitted single-top events & $0.48 \pm 0.21$ & $0.10 \pm 0.10$ & $0.20 \pm 0.10$ & $0.10 \pm 0.10$ & $0.10 \pm 0.10$ \\
\hline Fitted diboson events & $2.4 \pm 1.9$ & $0.7 \pm 0.7$ & $1.1 \pm 0.7$ & $0.4 \pm 0.4$ & $0.20 \pm 0.20$ \\
\hline Fitted $t \bar{t}+\mathrm{V}$ events & $0.13 \pm 0.04$ & $0.023 \pm 0.021$ & $0.089 \pm 0.021$ & $0.015 \pm 0.010$ & $0.0059 \pm 0.0021$ \\
\hline
\end{tabular}


TABLE XIV. Event yields and background-only fit results for the 9J SR. Each column corresponds to a bin in $m_{\text {eff }}$ $[\mathrm{GeV}]$. Uncertainties in the fitted background estimates combine statistical (in the simulated event yields) and systematic uncertainties. The uncertainties in this table are symmetrized for propagation purposes but truncated at zero to remain within the physical boundaries.

\begin{tabular}{lccc}
\hline \hline 9J & All $m_{\text {eff }}$ bins & {$[1000,1500]$} & $>1500[\mathrm{GeV}]$ \\
\hline Observed events & 10 & 6 & 4 \\
\hline Fitted bkg events & $7 \pm 4$ & $4.0 \pm 2.6$ & $3.1 \pm 1.6$ \\
\hline Fitted $W+$ jets events & $0.028_{-0.028}^{+0.057}$ & $0.006_{-0.006}^{+0.018}$ & $0.022_{-0.022}^{+0.043}$ \\
Fitted $t \bar{t}$ events & $0.7 \pm 0.6$ & $3.6 \pm 2.5$ & $2.3 \pm 1.6$ \\
Fitted single-top events & $0.30 \pm 0.17$ & $0.18_{-0.18}^{+0.27}$ & $0.5 \pm 0.4$ \\
Fitted $t \bar{t}+V$ events & $0.15_{-0.15}^{+0.16}$ & $0.14 \pm 0.09$ & $0.16 \pm 0.09$ \\
Fitted diboson events & $0.008_{-0.008}^{+0.011}$ & $0.029_{-0.029}^{+0.041}$ & $0.12 \pm 0.12$ \\
Fitted $Z+$ jets events & $\cdots$ & $0.008_{-0.008}^{+0.011}$ \\
\hline \hline
\end{tabular}

$$
f_{\text {closure }}=\frac{N_{\mathrm{SR}_{9 \mathrm{~J}}}^{\mathrm{sim}}}{N_{\mathrm{SR}_{9 \mathrm{~J}}}^{\mathrm{sim}, \mathrm{st}}}=\frac{N_{\mathrm{SR}_{9 \mathrm{~J}}}^{\mathrm{sim}} \cdot N_{\mathrm{CR}_{\mathrm{B}}}^{\mathrm{sim}}}{N_{\mathrm{CR}_{\mathrm{C}}}^{\mathrm{sim}} \cdot N_{\mathrm{CR}_{\mathrm{A}}}^{\mathrm{sim}}},
$$

where $N_{<\text {region }}^{\text {sim }}$ is the number of events in a given region as predicted by simulation while $N_{\mathrm{SR}_{9 \mathrm{~J}}}^{\text {sim,est }}$ is the estimated number of events in the signal region based on the simulation predictions in regions $\mathrm{A}, \mathrm{B}$, and $\mathrm{C}$. The estimated number of background events in the signal region can then be rewritten as

$$
\begin{aligned}
& N_{\mathrm{SR}_{9 \mathrm{~J}}}^{\text {est }}=f_{\text {closure }} \cdot \frac{N_{\mathrm{CR}_{\mathrm{A}}}^{\mathrm{obs}}}{N_{\mathrm{CR}_{\mathrm{B}}}^{\mathrm{obs}}} \cdot N_{\mathrm{CR}_{\mathrm{C}}}^{\mathrm{obs}} \\
& =N_{\mathrm{SR}_{9 \mathrm{~J}}}^{\operatorname{sim}} \cdot\left(\frac{N_{\mathrm{CR}_{\mathrm{A}}}}{N_{\mathrm{CR}_{\mathrm{B}}}} N_{\mathrm{CR}_{\mathrm{C}}}\right)^{\text {obs }} /\left(\frac{N_{\mathrm{CR}_{\mathrm{A}}}}{N_{\mathrm{CR}_{\mathrm{B}}}} N_{\mathrm{CR}_{\mathrm{C}}}\right)^{\mathrm{sim}}
\end{aligned}
$$

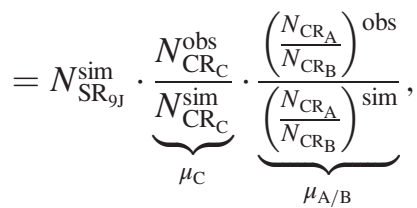

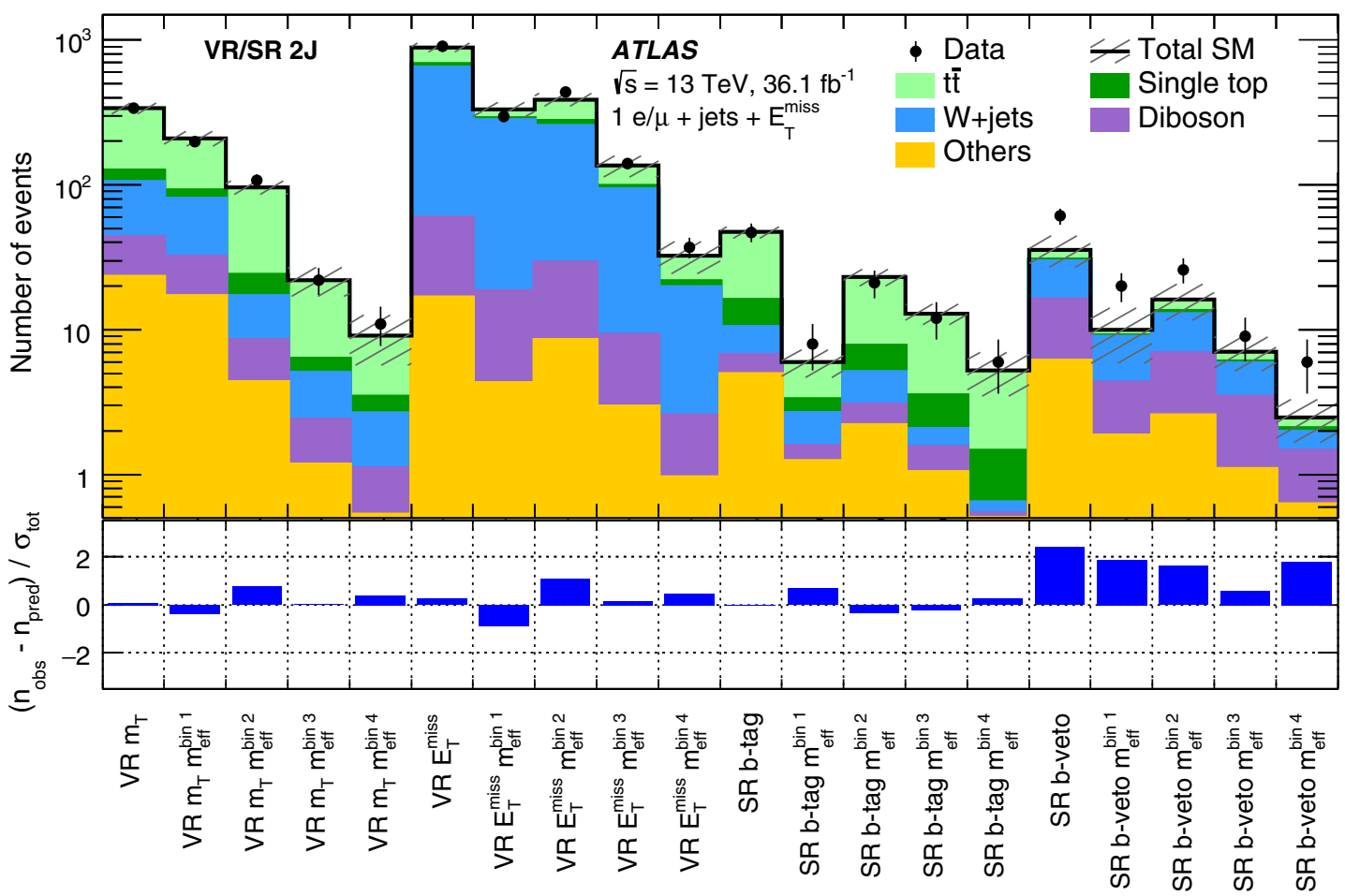

FIG. 3. Comparison of the observed and expected event yields in the $2 \mathrm{~J}$ validation and signal regions. Uncertainties in the background estimates include both the statistical (in the simulated event yields) and systematic uncertainties. Both the integrated regions and the regions for each $m_{\mathrm{eff}}$ bin are presented. 


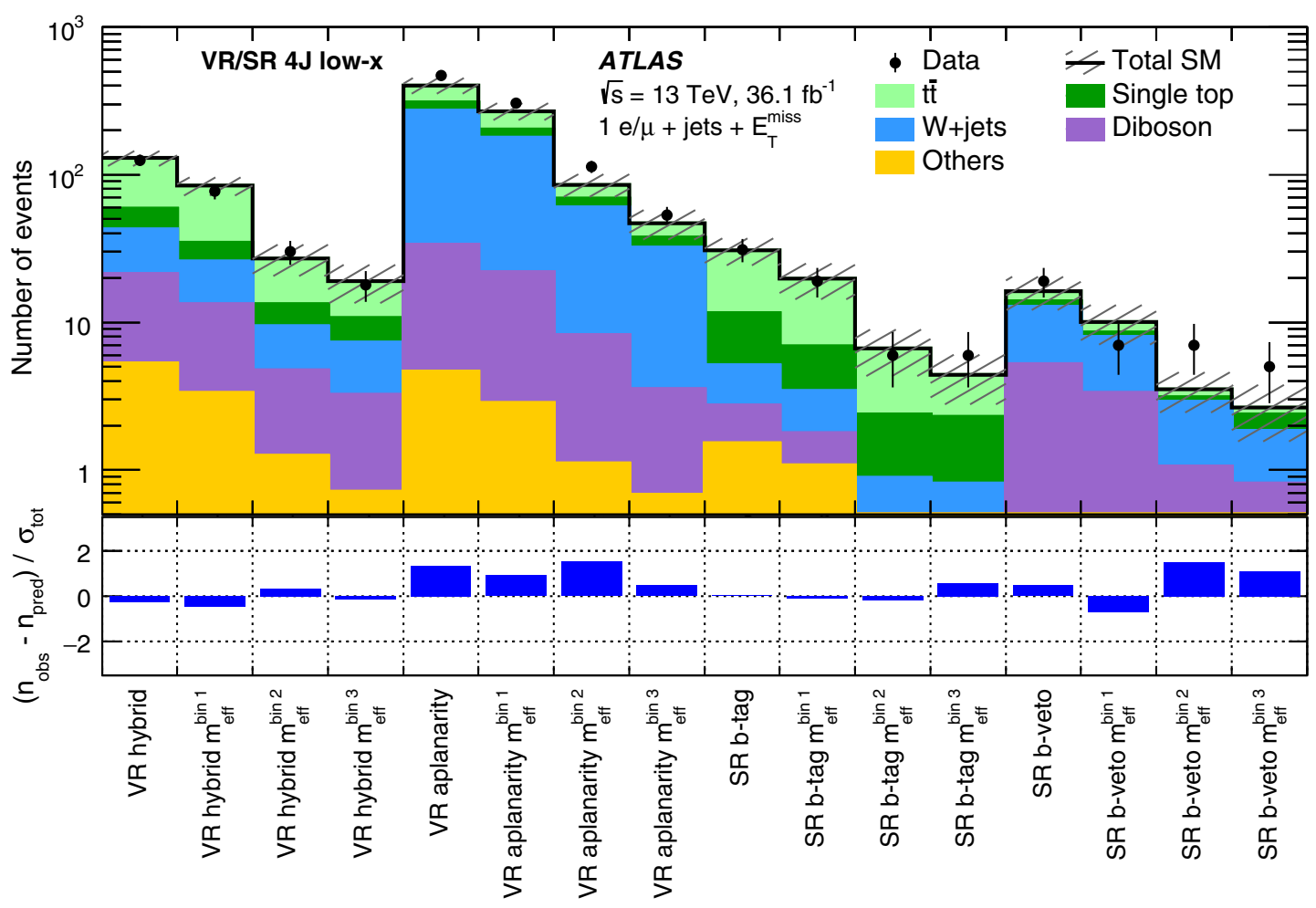

FIG. 4. Comparison of the observed and expected event yields in the $4 \mathrm{~J}$ low-x validation and signal regions. Uncertainties in the background estimates include both the statistical (in the simulated event yields) and systematic uncertainties. Both the integrated regions and the regions for each $m_{\mathrm{eff}}$ bin are presented.

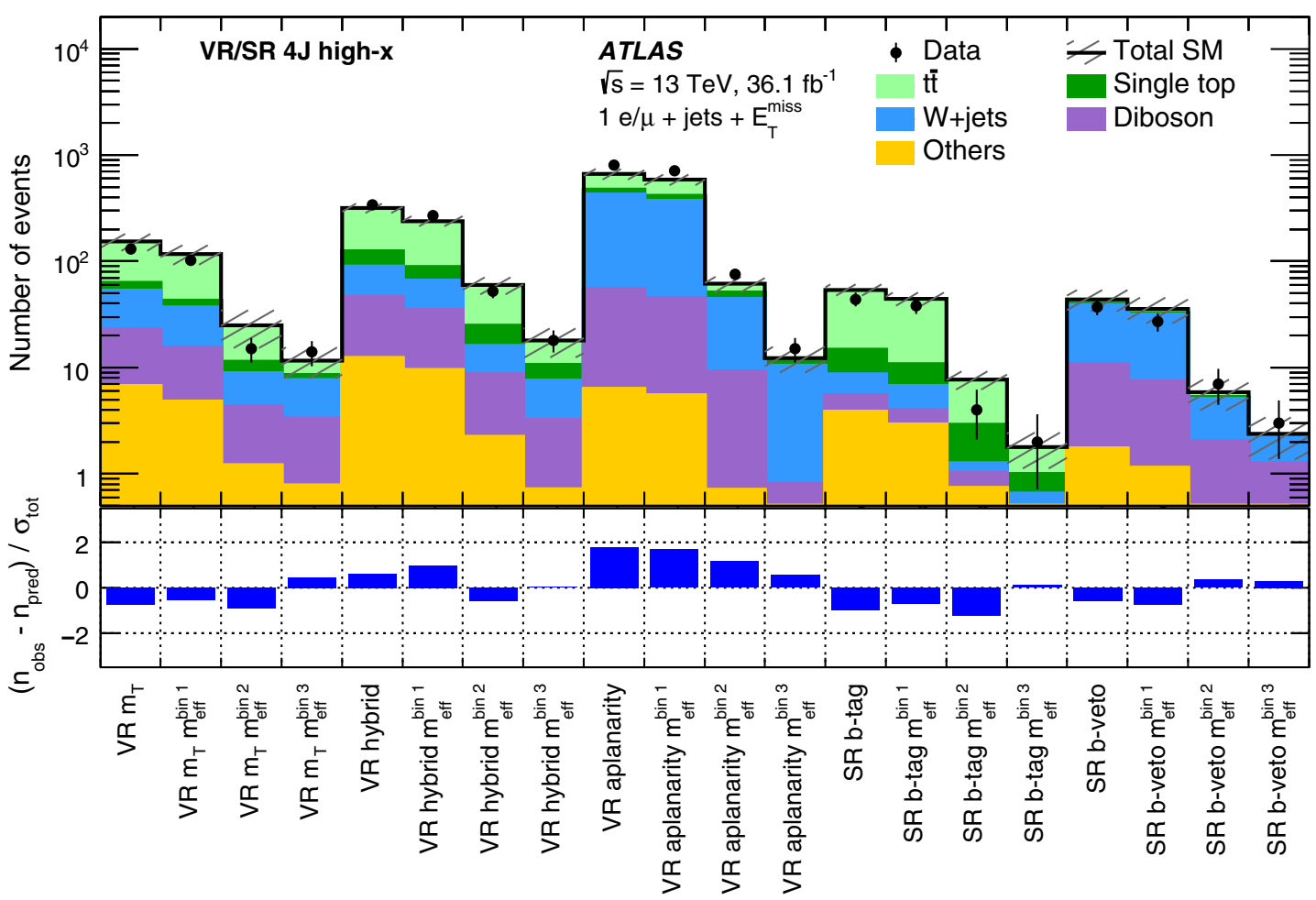

FIG. 5. Comparison of the observed and expected event yields in the 4J high-x validation and signal regions. Uncertainties in the background estimates include both the statistical (in the simulated event yields) and systematic uncertainties. Both the integrated regions and the regions for each $m_{\text {eff }}$ bin are presented. 


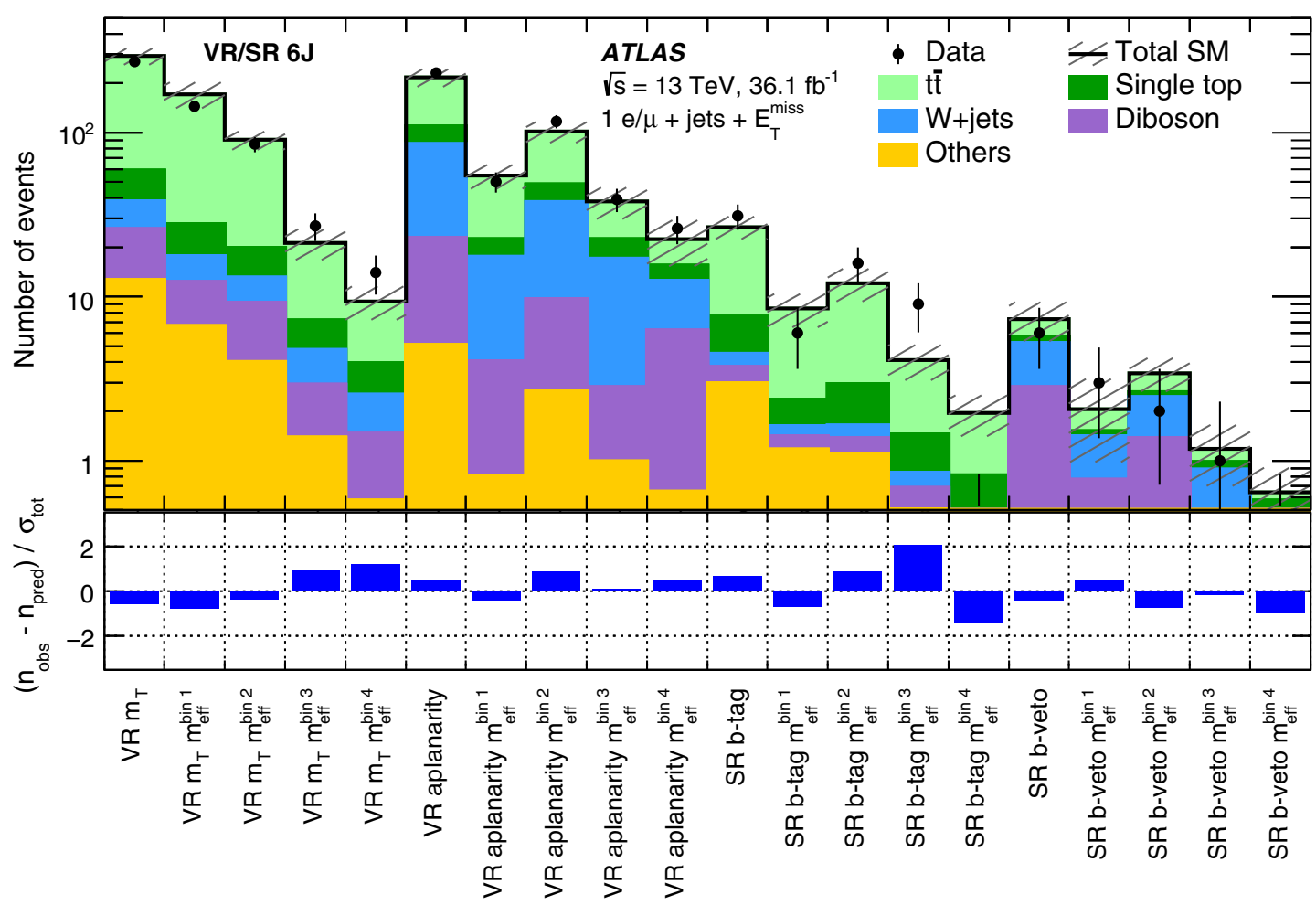

FIG. 6. Comparison of the observed and expected event yields in the $6 \mathrm{~J}$ validation and signal regions. Uncertainties in the background estimates include both the statistical (in the simulated event yields) and systematic uncertainties. Both the integrated regions and the regions for each $m_{\text {eff }}$ bin are presented.

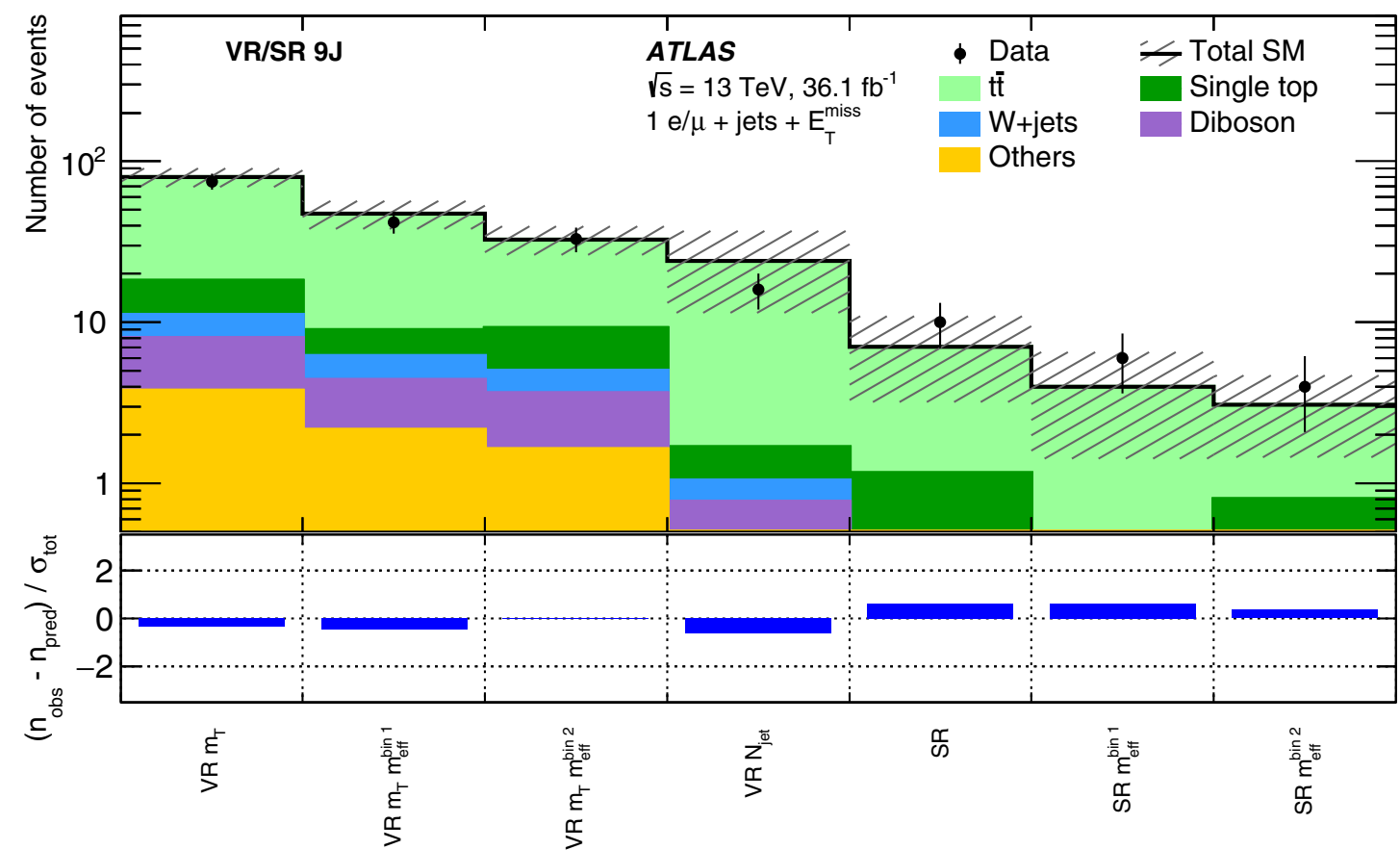

FIG. 7. Comparison of the observed and expected event yields in the 9J validation and signal regions. Uncertainties in the background estimates include both the statistical (in the simulated event yields) and systematic uncertainties. Both the integrated regions and the regions for each $m_{\text {eff }}$ bin are presented. 
where $N_{\langle\text {region }\rangle}^{\mathrm{obs}}$ is the observed number of events in a given region, $\mu_{\mathrm{C}}$ is the normalization parameter in region $\mathrm{C}$, and the normalization parameter $\mu_{\mathrm{A} / \mathrm{B}}$ is fitted simultaneously with the normalization $\mu_{\mathrm{B}}$ of the backgrounds in region $\mathrm{CR}_{\mathrm{B}}$ according to

$$
N_{\mathrm{CR}_{\mathrm{B}}}^{\mathrm{obs}}=N_{\mathrm{CR}_{\mathrm{B}}}^{\mathrm{sim}} \cdot \underbrace{\frac{N_{\mathrm{CR}_{\mathrm{B}}}^{\mathrm{obs}}}{N_{\mathrm{CR}_{\mathrm{B}}}^{\mathrm{sim}}}}_{\mu_{\mathrm{B}}} \text { and } N_{\mathrm{CR}_{\mathrm{A}}}^{\mathrm{obs}}=N_{\mathrm{CR}_{\mathrm{A}}}^{\mathrm{sim}} \cdot \underbrace{\frac{\left(\frac{N_{\mathrm{CR}_{\mathrm{A}}}}{N_{\mathrm{CR}_{\mathrm{B}}}}\right)^{\mathrm{obs}}}{\left(\frac{N_{\mathrm{CR}_{\mathrm{A}}}}{N_{\mathrm{CR}_{\mathrm{B}}}}\right)^{\operatorname{sim}}}}_{\mu_{\mathrm{A} / \mathrm{B}}} \underbrace{\frac{N_{\mathrm{CR}_{\mathrm{B}}}^{\mathrm{obs}}}{N_{\mathrm{CR}_{\mathrm{B}}}^{\mathrm{sim}}}}_{\mu_{\mathrm{B}}} .
$$

The control regions listed in Table VIII are optimized to provide a sufficient number of events in the backgrounds of interest, low contamination from the signal models considered, and a closure parameter $f_{\text {closure }}$ close to unity. All control regions are fitted simultaneously in two bins requiring either zero or at least one $b$-tagged signal jet to enrich the contributions from the $W+$ jets and top backgrounds, respectively. Therefore, the normalization factors $\mu_{\mathrm{B}}, \mu_{\mathrm{C}}$, and $\mu_{\mathrm{A} / \mathrm{B}}$ exist separately for the $W+$ jets and top backgrounds. The top backgrounds considered in the fit comprise $t \bar{t}$ as well as single-top production processes, which are treated with a common set of normalization parameters.

To validate that the fitted ratio of low- $m_{\mathrm{T}}$ to high- $m_{\mathrm{T}}$ events $\left(\mu_{\mathrm{A} / \mathrm{B}}\right)$ extrapolates to high values of $N_{\text {jet }}$, a validation region VR $m_{\mathrm{T}}$ with seven or eight jets and high $m_{\mathrm{T}}$ requirements is introduced. Similarly, a validation region VR $N_{\text {jet }}$ with at least nine jets and moderate $m_{\mathrm{T}}$ requirements is introduced to validate the extrapolation of the normalization factor $\mu_{\mathrm{C}}$ in region $\mathrm{CR}_{\mathrm{C}}$ to higher $m_{\mathrm{T}}$ values. Since the normalization factors for different jet multiplicities are expected to differ, a control region $\mathrm{CR}_{\mathrm{C}^{\prime}}$ along with its normalization factor $\left(\mu_{\mathrm{C}^{\prime}}\right)$ is introduced. This region is only used to obtain the background estimate in VR $m_{\mathrm{T}}$. Similarly, a control region $\mathrm{CR}_{\mathrm{A}^{\prime}}$ is constructed to obtain the normalization factor $\mu_{\mathrm{A}^{\prime} / \mathrm{B}}$ that is needed for the background estimation in validation
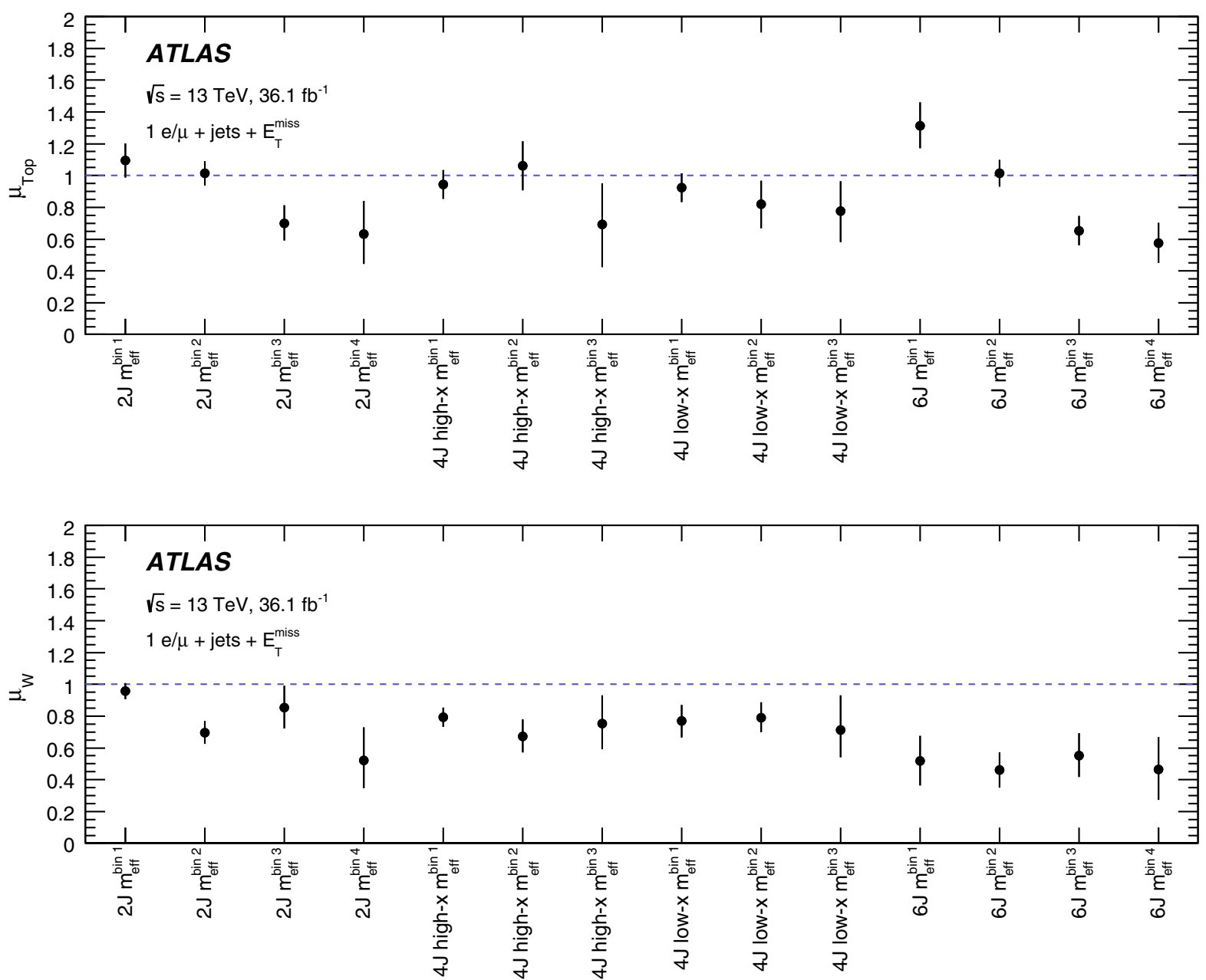

FIG. 8. Normalization factors $\mu_{\text {top }}$ and $\mu_{W}$ of the top and $W+$ jets backgrounds in the $m_{\text {eff }}$ bins of the $2 \mathrm{~J}, 4 \mathrm{~J}$ high-x, $4 \mathrm{~J}$ low-x, and $6 \mathrm{~J}$ SRs obtained with the background-only fit. The error bars correspond to the uncertainties as derived in the fit. 
region VR $N_{\text {jet }}$. The definition of the validation regions along with their corresponding control regions is given in Table VIII.

\section{SYSTEMATIC UNCERTAINTIES}

Experimental and theoretical sources of systematic uncertainty are described in this section. Their effects are evaluated for all simulated signal and background events.

The dominant experimental systematic effects are the uncertainties associated with the jet energy scale (JES) and resolution (JER) and with the $b$-tagging efficiency and mistagging rate. The impact of the jet-related uncertainties on the total background prediction ranges from $1.3 \%$ in the 6J SR to $18 \%$ in the 9J SR. Similarly, the impact of the uncertainties associated with the $b$-tagging procedure amounts to $1.9 \%$ in the $6 \mathrm{~J} \mathrm{SR}$ bins with at least one $b$-tagged jet and increases to $9.5 \%$ in the $6 \mathrm{~J}$ SR bins with no $b$-tagged jets. The simulation is reweighted to match the distribution of the average number of proton-proton interactions per bunch crossing $(\mu)$ observed in data. The uncertainty in $\mu$ is propagated by varying up and down the reweighting factor: it becomes relevant in the signal regions characterized by the highest jet multiplicities.
Uncertainties in the theoretical predictions and the modeling of simulated events are also considered. For the $W+$ jets and the $t \bar{t}$ and single top backgrounds, they affect the extrapolation from each $m_{\text {eff }}$ bin in the control regions to the corresponding bin in the signal regions. In the 9J SR the $f_{\text {closure }}$ parameter used in the background estimation in this channel is affected as well. For all the other background sources, they impact the inclusive cross section of each specific process, the acceptance of the analysis selection requirements and the shape of the $m_{\text {eff }}$ distribution in each SR.

An uncertainty stems from the choice of MC event generator modeling the $t \bar{t}$, single top, diboson and $W / Z+$ jets processes. For $t \bar{t}$ and single top, PowHEg-Box is compared with MG5_AMc@NLO [38] and the relative difference in the extrapolation factors is evaluated. For $W / Z+$ jets, the predictions from SHERPA are compared with MG5_AMc@NLO [38]. For dibosons, the event yield predictions from SHERPA are compared with POWHEG-BOX interfaced to PYTHIA. The impact of varying the amount of initial- and final-state radiation is evaluated for $t \bar{t}$ and single top production. Specific samples are used, with altered renormalization and factorization scales as well as parton shower and NLO radiation [50]. Moreover, the difference between the predictions from POWHEG-BOX interfaced to Pythia and to Herwig++ [96] is computed to estimate the

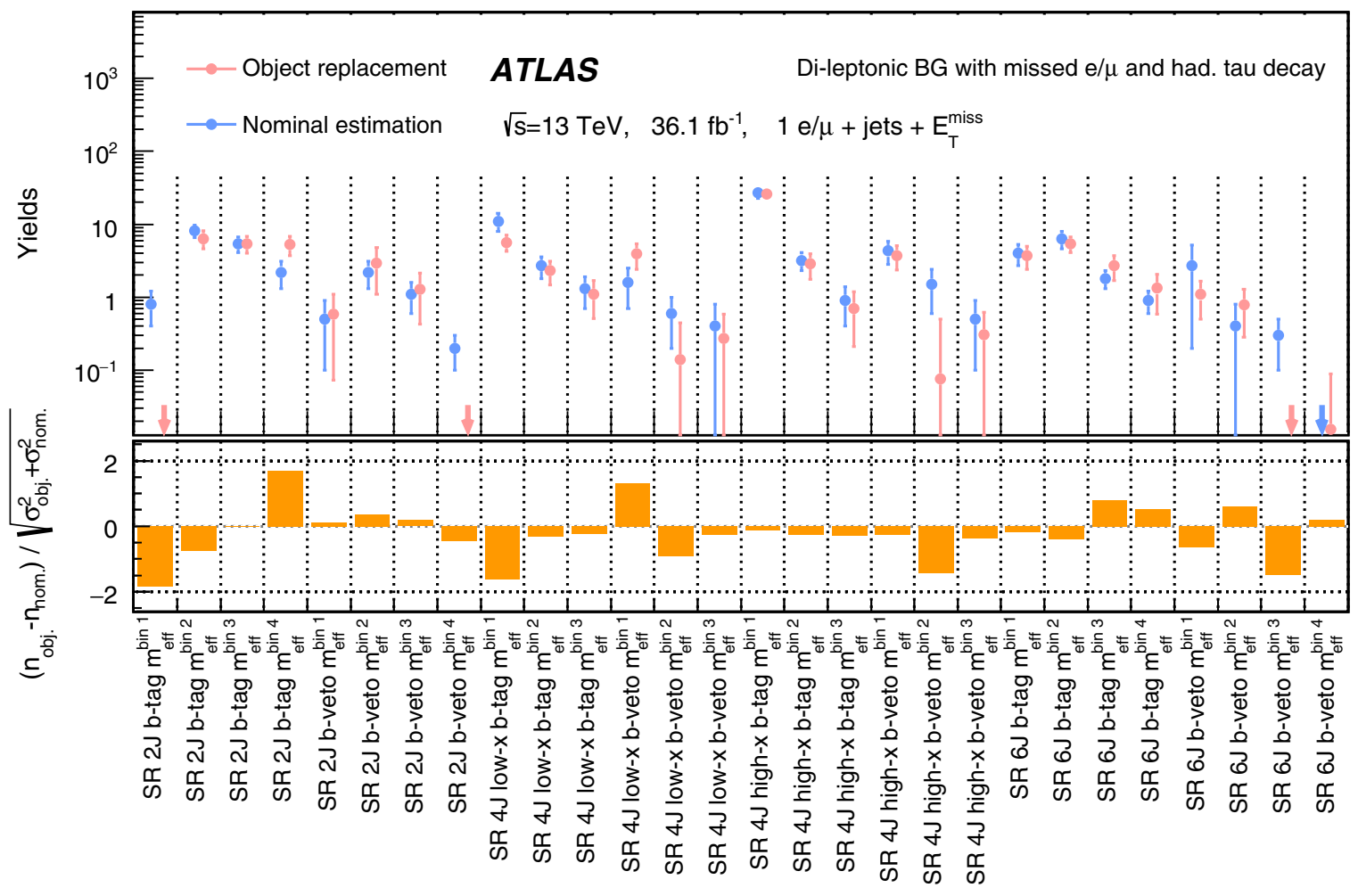

FIG. 9. Comparison of estimated Standard Model background event yield from $t \bar{t}$ processes with dileptonic decays in the 2J, 4J high-x, 4J low-x, and 6J SR using the nominal semi-data-driven background estimation technique and the alternative object-replacement technique described in Sec. VI. The error bands include both the statistical and systematic uncertainties. 
uncertainty associated with the parton shower modeling. For $W / Z+$ jets samples, the uncertainties in the renormalization, factorization, resummation scales and the matching scale between matrix elements and parton shower (CKKW-L) are evaluated by varying up and down by a factor of 2 the corresponding parameters in SHERPA. For $t \bar{t}$ and $W+$ jets samples, the uncertainties due to choosing the PDF set CT10 [52] are considered.

Inclusive $W W b b$ events generated using MG5_AMC@NLO [38] are compared to the sum of $t \bar{t}$ and $W t$ production, to assign an uncertainty to the interference effects between single top and $t \bar{t}$ production at NLO. The uncertainty in the inclusive $Z+$ jets cross section, amounting to 5\%, is accounted for [97]. An overall $6 \%$ systematic uncertainty in the inclusive cross section of diboson processes is also considered. In addition, the
SHERPA parameters controlling the renormalization, factorization, resummation and matching scales are varied by a factor of 2 to estimate the corresponding uncertainties. An uncertainty of $30 \%$ is assigned to the small contributions of $t \bar{t}+W / Z / W W$.

The total systematic uncertainty in the predicted background yields in the various signal regions ranges from $12 \%$ in the 2J SR bins with $\geq 1 b$-tagged jet, to $50 \%$ in the 9J SR. The largest uncertainties in the SR bins with $\geq 1$ $b$-tagged jet originate from the modeling of $t \bar{t}$ events and amount to $5 \%$ in the 2J SR, increasing to $40 \%$ in the $9 \mathrm{~J} \mathrm{SR}$. Similarly, in the SR bins where $b$-tagged jets are vetoed, the dominant source of systematic uncertainty is the modeling of $W+$ jets events, ranging from $9 \%$ in the $6 \mathrm{~J}$ SR to $20 \%$ in the $4 \mathrm{~J}$ low-x SR. Other important uncertainties are those associated with the finite size of the MC
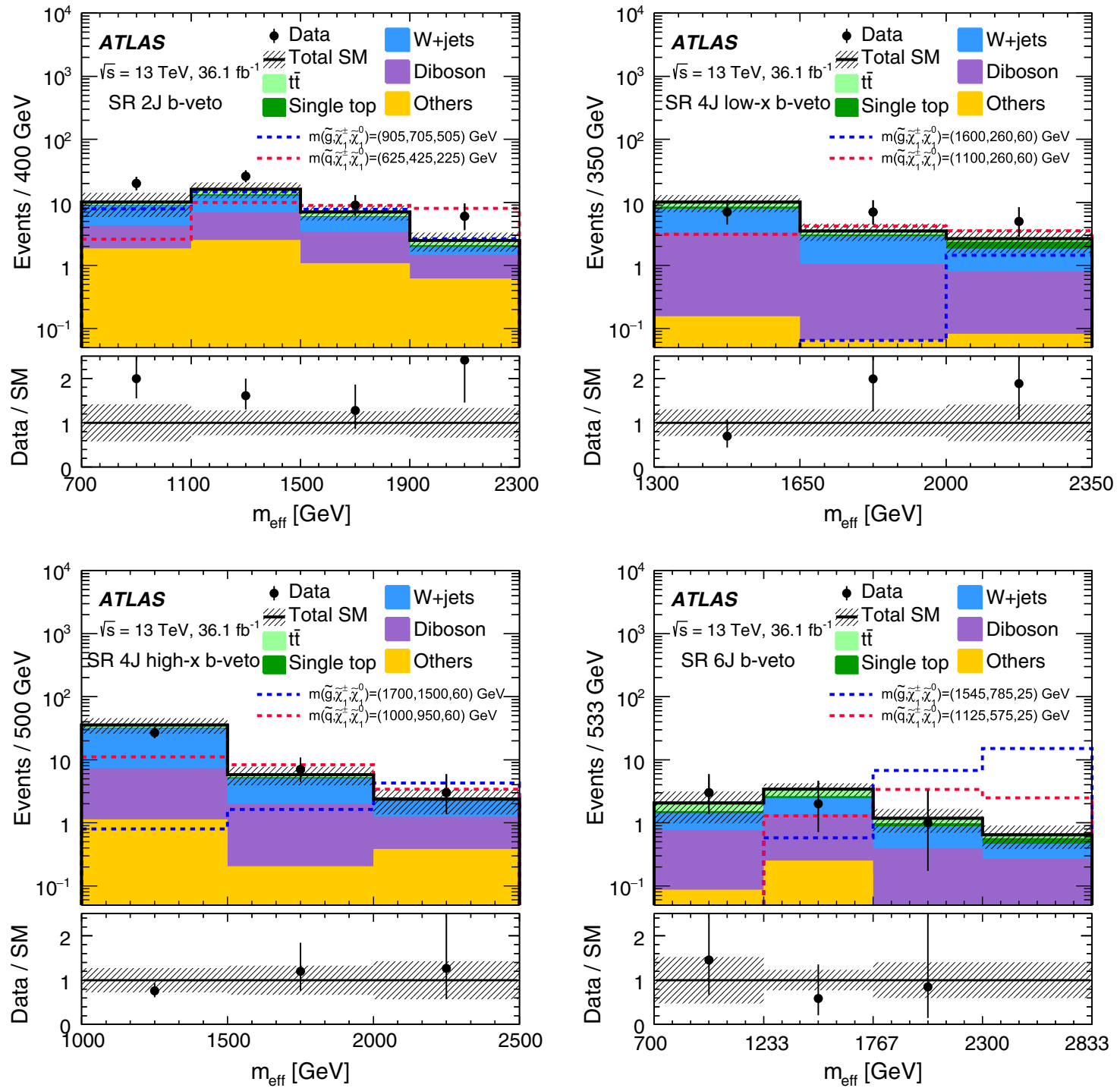

FIG. 10. The $m_{\text {eff }}$ distribution in $2 \mathrm{~J}$ (top left), 4J low-x (top right), 4J high-x (bottom left) and 6J (bottom right) $b$-veto signal regions after fit. The uncertainty bands plotted include all statistical and systematic uncertainties. The dashed lines stand for the benchmark signal samples. 
samples, which amount to $18 \%$ in the $6 \mathrm{~J} \mathrm{SR}$, and the theoretical uncertainties originating from the modeling of the diboson background, amounting to $26 \%$ in the $6 \mathrm{~J} \mathrm{SR}$. Tables IX-XI list the breakdown of the dominant systematic uncertainties in background estimates in the various signal regions.

For the signal processes, the modeling of initial-state radiation can be affected by sizable theoretical uncertainty. The uncertainties in the expected yields for SUSY signal models are estimated with variations of a factor of 2 to the MG5_AMC@NLO parameters corresponding to the renormalization, factorization and jet matching scales, and to the PYTHIA shower tune parameters. The overall uncertainties range from about $1 \%$ for signal models with large mass splitting between the gluino or squark, the chargino, and the neutralino, to $35 \%$ for models with very compressed mass spectra.

\section{RESULTS AND INTERPRETATION}

The statistical interpretation of the results is performed based on a profile likelihood method [98] using the HistFitter framework [99]. The likelihood function consists of a product of Poisson probability density functions for the signal and control regions that contribute to the fit. The inputs to the likelihood function are the observed numbers of data events and the expected numbers of signal and SM background events in each region. Three normalization factors, one for signal, one for $W+$ jets, one for $t \bar{t}$ and single top, are introduced to adjust the relative
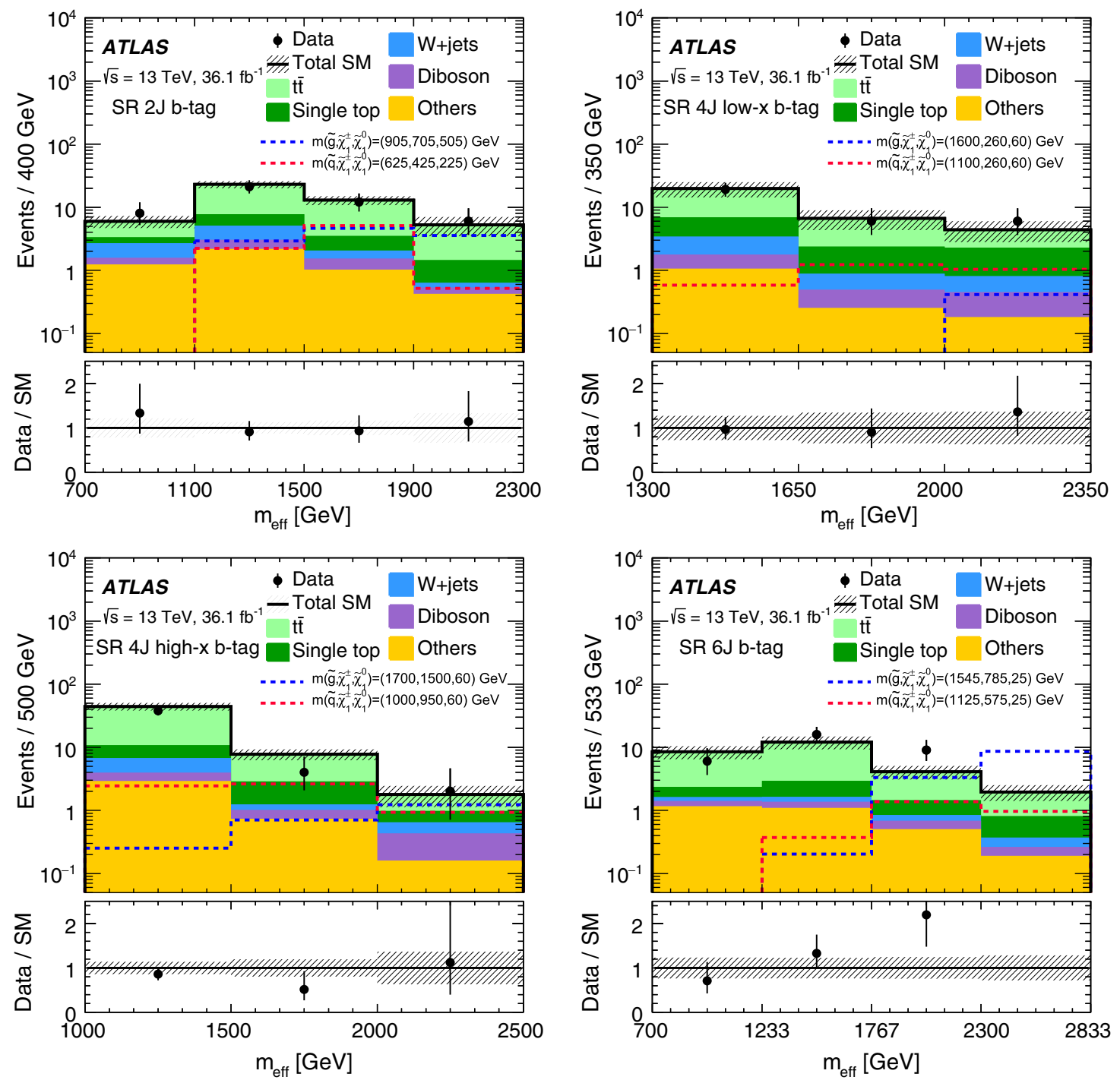

FIG. 11. The $m_{\text {eff }}$ distribution in $2 \mathrm{~J}$ (top left), $4 \mathrm{~J}$ low-x (top right), $4 \mathrm{~J}$ high-x (bottom left) and $6 \mathrm{~J}$ (bottom right) $b$-tag signal regions after fit. The uncertainty bands plotted include all statistical and systematic uncertainties. The dashed lines stand for the benchmark signal samples. 


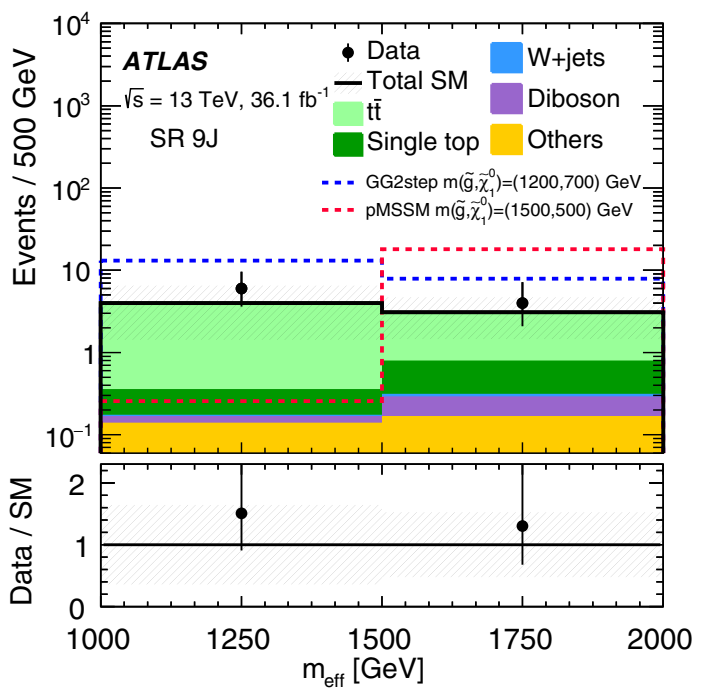

FIG. 12. The $m_{\text {eff }}$ distribution in the 9J signal region after fit. The uncertainty bands plotted include all statistical and systematic uncertainties. The dashed lines stand for the benchmark signal samples.

contributions of the main background and signal components. The small sources of SM background, i.e., diboson, $Z+$ jets and $t \bar{t}+V$, are estimated directly from simulation. The uncertainties are implemented in the fit as nuisance parameters, which are correlated between the SRs and the CRs. The systematic uncertainties described in Sec. VII are constrained by Gaussian probability density functions, while the statistical uncertainties are constrained by Poisson probability density functions.

The observed numbers of events in the signal regions are given in Tables XII-XIV, along with the SM background prediction as determined with the background-only fit. In a background-only fit, the data event yields in the CRs are used to determine the two background normalization factors: for $W+$ jets and for $t \bar{t}$ and single top production. The fit is independent of the observation in the SR, and does not consider signal contamination in the CRs. The above-mentioned signal normalization parameter is therefore not included in this fit configuration.
The compatibility of the observed and expected event yields in both the validation and signal regions is illustrated in Figures 3-7. No significant excess in data is observed over the SM prediction.

The top and $W+$ jets background normalization factors obtained for the 2J, 4J low-x, 4J high-x, and $6 \mathrm{~J}$ SRs are shown in bins of $m_{\text {eff }}$ in Fig. 8. A trend toward smaller normalization factors at large values of $m_{\text {eff }}$ is observed, which demonstrates the necessity of applying the same binning requirements in control and signal regions. The predicted event yields from $t \bar{t}$ events in which both top quarks decay semileptonically are cross-checked using the alternative object-replacement method described in Sec. VI. Figure 9 shows that the background estimates obtained from the two methods are consistent. Figures 10,11 show the $m_{\text {eff }}$ distribution in $2 \mathrm{~J}, 4 \mathrm{~J}$ low-X, $4 \mathrm{~J}$ high-x and $6 \mathrm{~J}$ in $b$-tag and $b$-veto signal regions after fit. Figure 12 shows the $m_{\text {eff }}$ distribution in 9J signal region after fit. The uncertainty bands plotted include all statistical and systematic uncertainties. The dashed lines stand for the benchmark signal samples.

Using the results of the background-only fit, a modelindependent limit fit is performed to test for the presence of any beyond-the-Standard-Model (BSM) physics processes that contribute to the SR ("disc" SR in Table II). The BSM signal is assumed to contribute only to the SR and not to the CRs, thus giving a conservative estimate of background in the SR. Observed $\left(S_{\mathrm{obs}}^{95}\right)$ and expected $\left(S_{\text {exp }}^{95}\right) 95 \%$ confidence level (C.L.) upper limits on the number of BSM signal events are derived using the $\mathrm{CL}_{\mathrm{s}}$ prescription [100]. Table XV presents these limits, together with the upper limits on the visible BSM cross section, $\langle\epsilon \sigma\rangle_{\mathrm{obs}}^{95}$, defined as the product of acceptance, selection efficiency and production cross section. The upper limits on the visible BSM cross section are calculated by dividing the observed upper limit on the beyond-SM events by the integrated luminosity of $36.1 \mathrm{fb}^{-1}$. Moreover, the discovery $p$-values are given. They quantify the probability under the background-only hypothesis to produce event yields greater than or equal to the observed data.

Additionally, the results are interpreted in the specific supersymmetric scenarios described in Sec. III using

TABLE XV. Results of the model-independent limit fits. For each SR, the observed 95\% C.L. upper limit on the visible cross ection $\left(\langle\epsilon \sigma\rangle_{\mathrm{obs}}^{95}\right)$, the observed $\left(S_{\mathrm{obs}}^{95}\right)$ and expected $\left(S_{\mathrm{exp}}^{95}\right) 95 \%$ C.L. upper limits on the BSM event yield, and the one-sided discovery $p$-value $[p(s=0)]$ are presented. The $p$-values are capped at 0.5 if fewer events than the fitted background estimate are observed.

\begin{tabular}{lccccccc}
\hline \hline $\mathrm{SR}_{\text {disc }}$ & 2J & 4J high-x & 4J low-x (gluino) & 4J low-x (squark) & 6J (gluino) & 6J (squark) & 9J \\
\hline Observed events & 80 & 16 & 24 & 50 & 0 & 28 \\
Fitted bkg events & $67 \pm 6$ & $17.7 \pm 2.7$ & $17.2 \pm 3.2$ & $47 \pm 7$ & $2.6 \pm 0.6$ & $23.4 \pm 3.1$ & $3.1 \pm 1.6$ \\
$\langle\epsilon \sigma\rangle_{\text {obs }}^{95}[\mathrm{fb}]$ & 0.92 & 0.27 & 0.50 & 0.62 & 0.08 & 0.46 & 0.20 \\
$S_{\text {obs }}^{95}$ & 33.1 & 9.8 & 18.0 & 22.5 & 3.0 & 16.6 \\
$S_{\text {exp }}^{95}$ & $21.6_{-5.6}^{+9.2}$ & $10.8_{-3.0}^{+3.7}$ & $11.8_{-2.7}^{+4.8}$ & $19.9_{-5.6}^{+7.5}$ & $4.5_{-1.0}^{+1.8}$ & $12.7_{-4.0}^{+5.0}$ & $6.0_{-1.2}^{+2.2}$ \\
$p(s=0)$ & 0.10 & 0.50 & 0.10 & 0.35 & 0.50 & 0.21 \\
\hline \hline
\end{tabular}



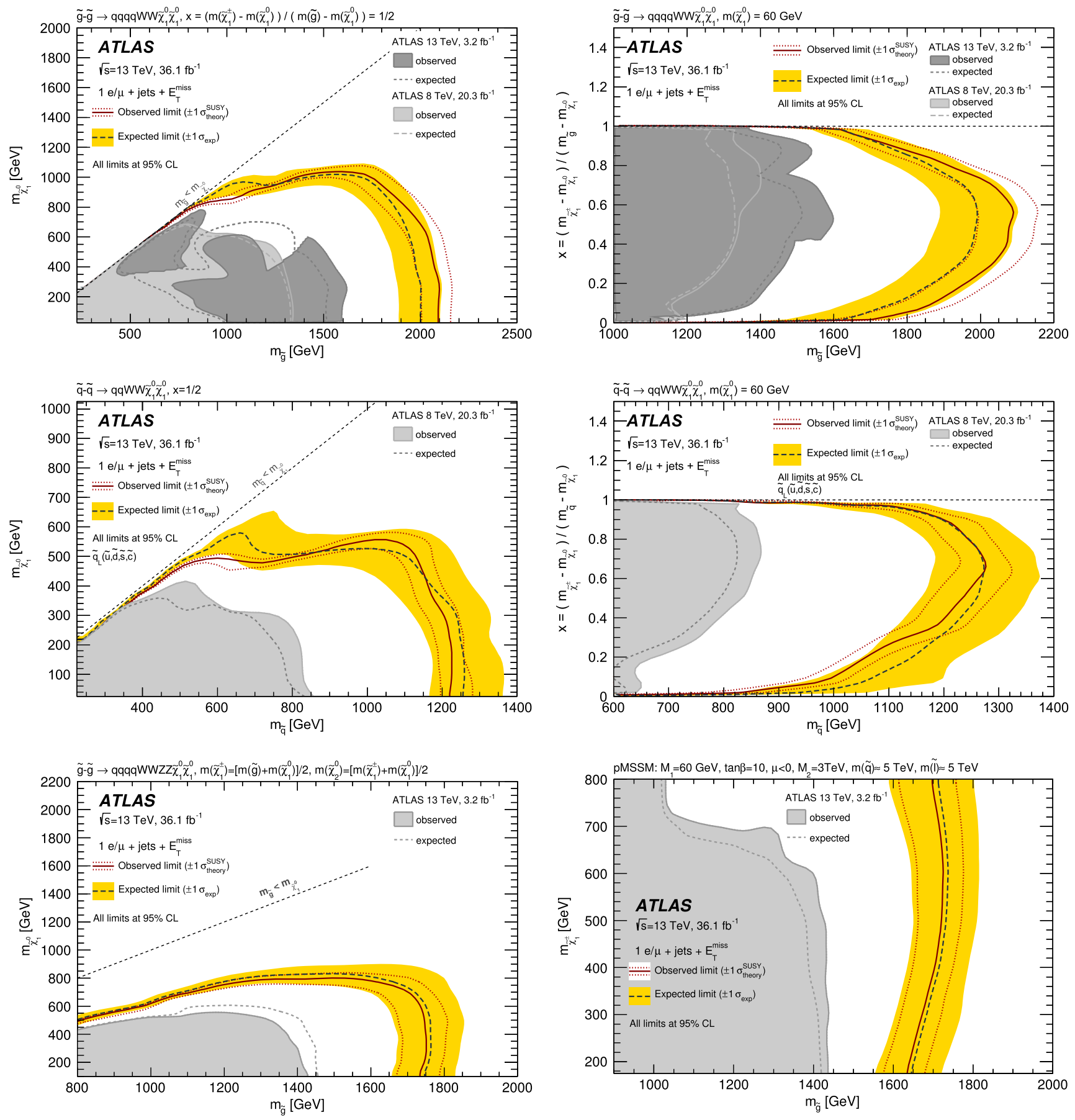

FIG. 13. Exclusion contours for gluino one-step $x=1 / 2$ (top left), gluino one-step variable- $x$ (top right), squark one-step $x=1 / 2$ (middle left) and squark one-step variable- $x$ (middle right), gluino two-step (bottom left), and the pMSSM scenario (bottom right). The red solid line corresponds to the observed limit with the red dotted lines indicating the $\pm 1 \sigma$ variation of this limit due to the effect of theoretical scale and PDF uncertainties in the signal cross section. The dark gray dashed line indicates the expected limit with the yellow band representing the $\pm 1 \sigma$ variation of the median expected limit due to the experimental and theoretical uncertainties. For reference, exclusion bounds from previous searches with $20.3 \mathrm{fb}^{-1}$ at $8 \mathrm{TeV}$ center-of-mass energy [28] and $3.2 \mathrm{fb}^{-1}$ at $13 \mathrm{TeV}$ center-of-mass energy [22,30] are overlaid where applicable by the gray area (the observed limit is shown by the solid line, while the dashed line shows the expected limit).

model-dependent limit fits. A model-dependent limit fit takes the data event yields in multiple, statistically independent SRs and their associated CRs to compute an upper limit on the cross section of a targeted SUSY model. The fit includes the expected signal contributions to the SRs and to the CRs, scaled by a floating signal normalization factor. The background normalization factors are also determined simultaneously in the fit. 
The sparticle mass in a specific SUSY model can be excluded if the upper limit of the signal normalization factor obtained in the fit is smaller than unity.

For the gluino/squark one-step models, a model-dependent fit is performed over all bins of the $2 \mathrm{~J}, 4 \mathrm{~J}$ high- $\mathrm{x}, 4 \mathrm{~J}$ low-x, and $6 \mathrm{~J}$ SRs. An independent set of background normalization factors are allocated for each bin of each SR ("excl" SR in Table II) and its associated CRs. Figure 13 (top and middle) shows the observed and expected exclusion bounds at $95 \%$ C.L. for the one-step simplified models with gluino and squark production. Gluino masses up to $2.1 \mathrm{TeV}$ and squark masses up to $1.25 \mathrm{TeV}$ are excluded.

Figure 13 (bottom) shows the exclusion contours of the 9J SR (Table III) for the gluino two-step as well as the pMSSM scenario described in Sec. III. In both cases the limits reach well beyond $1.7 \mathrm{TeV}$ in gluino mass.

\section{CONCLUSION}

A search for the pair production of squarks and gluinos in proton-proton collisions provided by the LHC at a center-of-mass energy of $\sqrt{s}=13 \mathrm{TeV}$ has been performed by the ATLAS Collaboration. Events containing one isolated electron or muon, two or more jets, and large missing transverse momentum are selected in the data collected in 2015 and 2016, corresponding to an integrated luminosity of $36.1 \mathrm{fb}^{-1}$. No significant excess over the Standard Model background prediction is observed. Exclusion limits are set at 95\% C.L. on generic new phenomena beyond the Standard Model and on sparticle masses in a number of specific SUSY scenarios. The exclusion limits extend up to $2.1 \mathrm{TeV}$ in gluino mass and $1.25 \mathrm{TeV}$ in squark mass thus significantly improving on the sensitivity of previous searches in this final state.

\section{ACKNOWLEDGMENTS}

We thank CERN for the very successful operation of the LHC, as well as the support staff from our institutions without whom ATLAS could not be operated efficiently. We acknowledge the support of ANPCyT, Argentina;
YerPhI, Armenia; ARC, Australia; BMWFW and FWF, Austria; ANAS, Azerbaijan; SSTC, Belarus; $\mathrm{CNPq}$ and FAPESP, Brazil; NSERC, NRC and CFI, Canada; CERN; CONICYT, Chile; CAS, MOST and NSFC, China; COLCIENCIAS, Colombia; MSMT CR, MPO CR and VSC CR, Czech Republic; DNRF and DNSRC, Denmark; IN2P3-CNRS, CEA-DSM/IRFU, France; SRNSF, Georgia; BMBF, HGF, and MPG, Germany; GSRT, Greece; RGC, Hong Kong SAR, China; ISF, I-CORE and Benoziyo Center, Israel; INFN, Italy; MEXT and JSPS, Japan; CNRST, Morocco; NWO, Netherlands; RCN, Norway; MNiSW and NCN, Poland; FCT, Portugal; MNE/IFA, Romania; MES of Russia and NRC KI, Russian Federation; JINR; MESTD, Serbia; MSSR, Slovakia; ARRS and MIZŠ, Slovenia; DST/NRF, South Africa; MINECO, Spain; SRC and Wallenberg Foundation, Sweden; SERI, SNSF and Cantons of Bern and Geneva, Switzerland; MOST, Taiwan; TAEK, Turkey; STFC, United Kingdom; DOE and NSF, United States of America. In addition, individual groups and members have received support from BCKDF, the Canada Council, CANARIE, CRC, Compute Canada, FQRNT, and the Ontario Innovation Trust, Canada; EPLANET, ERC, ERDF, FP7, Horizon 2020 and Marie Skłodowska-Curie Actions, European Union; Investissements d'Avenir Labex and Idex, ANR, Région Auvergne and Fondation Partager le Savoir, France; DFG and AvH Foundation, Germany; Herakleitos, Thales and Aristeia programmes co-financed by EU-ESF and the Greek NSRF; BSF, GIF and Minerva, Israel; BRF, Norway; CERCA Programme Generalitat de Catalunya, Generalitat Valenciana, Spain; the Royal Society and Leverhulme Trust, United Kingdom. The crucial computing support from all WLCG partners is acknowledged gratefully, in particular from CERN, the ATLAS Tier-1 facilities at TRIUMF (Canada), NDGF (Denmark, Norway, Sweden), CC-IN2P3 (France), KIT/ GridKA (Germany), INFN-CNAF (Italy), NL-T1 (Netherlands), PIC (Spain), ASGC (Taiwan), RAL (UK) and BNL (USA), the Tier-2 facilities worldwide and large non-WLCG resource providers. Major contributors of computing resources are listed in Ref. [101].
[1] Yu. A. Golfand and E. P. Likhtman, Extension of the algebra of Poincare Group generators and violation of p invariance, Pis'ma Zh. Eksp. Teor. Fiz. 13, 452 (1971) [JETP Lett. 13, 323 (1971)].

[2] D. V. Volkov and V. P. Akulov, Is the neutrino a Goldstone particle?, Phys. Lett. 46B, 109 (1973).

[3] J. Wess and B. Zumino, Supergauge transformations in four dimensions, Nucl. Phys. B70, 39 (1974).
[4] J. Wess and B. Zumino, Supergauge invariant extension of quantum electrodynamics, Nucl. Phys. B78, 1 (1974).

[5] S. Ferrara and B. Zumino, Supergauge invariant YangMills theories, Nucl. Phys. B79, 413 (1974).

[6] A. Salam and J. A. Strathdee, Supersymmetry and nonAbelian gauges, Phys. Lett. 51B, 353 (1974).

[7] P. Fayet, Supersymmetry and weak, electromagnetic and strong interactions, Phys. Lett. 64B, 159 (1976). 
[8] P. Fayet, Spontaneously Broken supersymmetric theories of weak, electromagnetic and strong interactions, Phys. Lett. 69B, 489 (1977).

[9] N. Sakai, Naturalness in supersymmetric GUTS, Z. Phys. C 11, 153 (1981).

[10] S. Dimopoulos, S. Raby, and F. Wilczek, Supersymmetry and the scale of unification, Phys. Rev. D 24, 1681 (1981).

[11] L. E. Ibañez and G. G. Ross, Low-energy predictions in supersymmetric grand unified theories, Phys. Lett. 105B, 439 (1981).

[12] S. Dimopoulos and H. Georgi, Softly broken supersymmetry and SU(5), Nucl. Phys. B193, 150 (1981).

[13] G. R. Farrar and P. Fayet, Phenomenology of the production, decay, and detection of new hadronic states associated with supersymmetry, Phys. Lett. 76B, 575 (1978).

[14] H. Goldberg, Constraint on the Photino Mass from Cosmology, Phys. Rev. Lett. 50, 1419 (1983); Erratum, Phys. Rev. Lett. 103, 099905(E) (2009).

[15] J. R. Ellis, J. S. Hagelin, D. V. Nanopoulos, K. A. Olive, and M. Srednicki, Supersymmetric relics from the big bang, Nucl. Phys. B238, 453 (1984).

[16] A. Djouadi et al., The minimal supersymmetric standard model: Group summary report, arXiv:hep-ph/9901246.

[17] C. F. Berger, J. S. Gainer, J. L. Hewett, and T. G. Rizzo, Supersymmetry without prejudice, J. High Energy Phys. 02 (2009) 023.

[18] J. Alwall, M.-P. Le, M. Lisanti, and J. G. Wacker, Searching for directly decaying gluinos at the Tevatron, Phys. Lett. B 666, 34 (2008).

[19] J. Alwall, P. Schuster, and N. Toro, Simplified models for a first characterization of new physics at the LHC, Phys. Rev. D 79, 075020 (2009).

[20] D. Alves et al., Simplified models for LHC new physics searches, J. Phys. G 39, 105005 (2012).

[21] ATLAS Collaboration, Search for squarks and gluinos in events with isolated leptons, jets and missing transverse momentum at $\sqrt{s}=8 \mathrm{TeV}$ with the ATLAS detector, J. High Energy Phys. 04 (2015) 116.

[22] ATLAS Collaboration, Search for gluinos in events with an isolated lepton, jets and missing transverse momentum at $\sqrt{s}=13 \mathrm{TeV}$ with the ATLAS detector, Eur. Phys. J. C 76, 565 (2016).

[23] CMS Collaboration, Search for new physics in events with same-sign dileptons and jets in pp collisions at $\sqrt{s}=8 \mathrm{TeV}$, J. High Energy Phys. 01 (2014) 163; Erratum, J. High Energy Phys. 01 (2015) 014.

[24] CMS Collaboration, Search for new physics in the multijet and missing transverse momentum final state in protonproton collisions at $\sqrt{s}=8 \mathrm{TeV}, \mathrm{J}$. High Energy Phys. 06 (2014) 055.

[25] CMS Collaboration, Search for supersymmetry in events with one lepton and multiple jets in proton-proton collisions at $\sqrt{s}=13 \mathrm{TeV}$, Phys. Rev. D 95, 012011 (2017).

[26] CMS Collaboration, Search for physics beyond the standard model in events with two leptons of same sign, missing transverse momentum, and jets in proton-proton collisions at $\sqrt{s}=13 \mathrm{TeV}$, Eur. Phys. J. C 77, 578 (2017).

[27] CMS Collaboration, Search for supersymmetry in multijet events with missing transverse momentum in proton-proton collisions at $13 \mathrm{TeV}$, Phys. Rev. D 96, 032003 (2017).

[28] ATLAS Collaboration, Summary of the searches for squarks and gluinos using $\sqrt{s}=8 \mathrm{TeV}$ pp collisions with the ATLAS experiment at the LHC, J. High Energy Phys. 10 (2015) 054.

[29] ATLAS Collaboration, Search for squarks and gluinos in final states with jets and missing transverse momentum at $\sqrt{s}=13 \mathrm{TeV}$ with the ATLAS detector, Eur. Phys. J. C 76, 392 (2016),

[30] ATLAS Collaboration, Search for new phenomena in final states with large jet multiplicities and missing transverse momentum with ATLAS using $\sqrt{s}=13 \mathrm{TeV}$ protonproton collisions, Phys. Lett. B 757, 334 (2016).

[31] ATLAS Collaboration, Search for supersymmetry in final states with two same-sign or three leptons and jets using $36 \mathrm{fb}^{-1}$ of $\sqrt{s}=13 \mathrm{TeV} p p$ collision data with the ATLAS detector, J. High Energy Phys. 09 (2017) 084.

[32] ATLAS Collaboration, The ATLAS experiment at the CERN large hadron collider, J. Instrum. 3, S08003 (2008).

[33] ATLAS Collaboration, Report No. ATLAS-TDR-19, 2010, https://cds.cern.ch/record/1291633; Report No. ATLAS-TDR-19-ADD-1， 2012， https://cds.cern.ch/record/ 1451888.

[34] ATLAS Collaboration, Performance of the ATLAS Trigger System in 2015, Eur. Phys. J. C 77, 317 (2017).

[35] ATLAS Collaboration, Summary of the ATLAS experiment's sensitivity to supersymmetry after LHC Run 1-interpreted in the phenomenological MSSM, J. High Energy Phys. 10 (2015) 134.

[36] B. C. Allanach, SOFTSUSY: A program for calculating supersymmetric spectra, Comput. Phys. Commun. 143, 305 (2002).

[37] A. Djouadi, M. Mühlleitner, and M. Spira, Decays of supersymmetric particles: The Program SUSY-HIT (SUspect-SdecaY-Hdecay-InTerface), Acta Phys. Pol. B 38, 635 (2007).

[38] J. Alwall, R. Frederix, S. Frixione, V. Hirschi, F. Maltoni, O. Mattelaer, H.-S. Shao, T. Stelzer, P. Torrielli, and M. Zaro, The automated computation of tree-level and nextto-leading order differential cross sections, and their matching to parton shower simulations, J. High Energy Phys. 07 (2014) 079.

[39] T. Sjöstrand, S. Mrenna, and P.Z. Skands, A Brief Introduction to PYTHIA 8.1, Comput. Phys. Commun. 178, 852 (2008).

[40] L. Lönnblad and S. Prestel, Matching tree-level matrix elements with interleaved showers, J. High Energy Phys. 03 (2012) 019.

[41] ATLAS Collaboration, Report No. ATL-PHYS-PUB2014-021, 2014, https://cdsweb.cern.ch/record/1966419.

[42] R. D. Ball et al., Parton distributions with LHC data, Nucl. Phys. B867, 244 (2013).

[43] D. J. Lange, The EvtGen particle decay simulation package, Nucl. Instrum. Methods Phys. Res., Sect. A 462, 152 (2001).

[44] W. Beenakker, R. Hopker, M. Spira, and P. Zerwas, Squark and gluino production at hadron colliders, Nucl. Phys. B492, 51 (1997). 
[45] A. Kulesza and L. Motyka, Threshold resummation for squark-antisquark and gluino-pair production at the LHC, Phys. Rev. Lett. 102, 111802 (2009).

[46] A. Kulesza and L. Motyka, Soft gluon resummation for the production of gluino-gluino and squark-antisquark pairs at the LHC, Phys. Rev. D 80, 095004 (2009).

[47] W. Beenakker, S. Brensing, M. Krämer, A. Kulesza, E. Laenen, and I. Niessen, Soft-gluon resummation for squark and gluino hadroproduction, J. High Energy Phys. 12 (2009) 041.

[48] W. Beenakker, S. Brensing, M. Krämer, A. Kulesza, E. Laenen, L. Motyka, and I. Niessen, Squark and gluino hadroproduction, Int. J. Mod. Phys. A 26, 2637 (2011).

[49] C. Borschensky, M. Krämer, A. Kulesza, M. Mangano, S. Padhi, T. Plehn, and X. Portell, Squark and gluino production cross sections in $\mathrm{pp}$ collisions at $\sqrt{s}=13$, 14, 33 and 100 TeV, Eur. Phys. J. C 74, 3174 (2014).

[50] ATLAS Collaboration, Report No. ATL-PHYS-PUB2016-004, 2016, https://cds.cern.ch/record/2120417.

[51] S. Alioli, P. Nason, C. Oleari, and E. Re, A general framework for implementing NLO calculations in shower Monte Carlo programs: The POWHEG BOX, J. High Energy Phys. 06 (2010) 043.

[52] H.-L. Lai, M. Guzzi, J. Huston, Z. Li, P. M. Nadolsky, J. Pumplin, and C.-P. Yuan, New parton distributions for collider physics, Phys. Rev. D 82, 074024 (2010).

[53] P. Artoisenet, R. Frederix, O. Mattelaer, and R. Rietkerk, Automatic spin-entangled decays of heavy resonances in Monte Carlo simulations, J. High Energy Phys. 03 (2013) 015.

[54] T. Sjöstrand, S. Mrenna, and P.Z. Skands, PYTHIA 6.4 Physics and Manual, J. High Energy Phys. 05 (2006) 026.

[55] J. Pumplin, D. R. Stump, J. Huston, H.-L. Lai, P. Nadolsky, and W.-K. Tung, New generation of parton distributions with uncertainties from global QCD analysis, J. High Energy Phys. 07 (2002) 012.

[56] P. Z. Skands, Tuning Monte Carlo generators: The Perugia tunes, Phys. Rev. D 82, 074018 (2010).

[57] M. Czakon and A. Mitov, Top++: A program for the calculation of the top-pair cross-section at hadron colliders, Comput. Phys. Commun. 185, 2930 (2014).

[58] N. Kidonakis, Two-loop soft anomalous dimensions for single top quark associated production with a W- or $\mathrm{H}$-, Phys. Rev. D 82, 054018 (2010).

[59] P. Kant, O. M. Kind, T. Kintscher, T. Lohse, T. Martini, S. Mölbitz, P. Rieck, and P. Uwer, HATHOR for single top-quark production: Updated predictions and uncertainty estimates for single top-quark production in hadronic collisions, Comput. Phys. Commun. 191, 74 (2015).

[60] ATLAS Collaboration, Report No. ATL-PHYS-PUB2016-003, 2016, https://cds.cern.ch/record/2120133.

[61] T. Gleisberg, S. Höche, F. Krauss, M. Schönherr, S. Schumann, F. Siegert, and J. Winter, Event generation with SHERPA 1.1, J. High Energy Phys. 02 (2009) 007.

[62] T. Gleisberg and S. Höche, Comix, a new matrix element generator, J. High Energy Phys. 12 (2008) 039.

[63] F. Cascioli, P. Maierhofer, and S. Pozzorini, Scattering Amplitudes with Open Loops, Phys. Rev. Lett. 108, 111601 (2012).
[64] S. Schumann and F. Krauss, A Parton shower algorithm based on Catani-Seymour dipole factorisation, J. High Energy Phys. 03 (2008) 038.

[65] S. Höche, F. Krauss, M. Schönherr, and F. Siegert, QCD matrix elements + parton showers: The NLO case, J. High Energy Phys. 04 (2013) 027.

[66] R. D. Ball et al., Parton distributions for the LHC Run II, J. High Energy Phys. 04 (2015) 040.

[67] R. Gavin, Y. Li, F. Petriello, and S. Quackenbush, FEWZ 2.0: A code for hadronic $\mathrm{Z}$ production at next-to-next-toleading order, Comput. Phys. Commun. 182, 2388 (2011).

[68] ATLAS Collaboration, Report No. ATL-PHYS-PUB2016-002, 2016, https://cds.cern.ch/record/2119986.

[69] ATLAS Collaboration, Report No. ATL-PHYS-PUB2016-005, 2016, https://cds.cern.ch/record/2120826.

[70] A. Lazopoulos, T. McElmurry, K. Melnikov, and F. Petriello, Next-to-leading order QCD corrections to $t \bar{t} Z$ production at the LHC, Phys. Lett. B 666, 62 (2008).

[71] J. M. Campbell and R. K. Ellis, $t \bar{t} W^{ \pm}$production and decay at NLO, J. High Energy Phys. 07 (2012) 052.

[72] ATLAS Collaboration, The ATLAS simulation infrastructure, Eur. Phys. J. C 70, 823 (2010).

[73] S. Agostinelli et al., GEANT4: A simulation toolkit, Nucl. Instrum. Methods Phys. Res., Sect. A 506, 250 (2003).

[74] ATLAS Collaboration, Report No. ATL-PHYS-PUB2010-013, 2010, https://cds.cern.ch/record/1300517.

[75] ATLAS Collaboration, Report No. ATL-PHYS-PUB2012-003, 2012, https://cds.cern.ch/record/1474107.

[76] A. D. Martin, W. J. Stirling, R. S. Thorne, and G. Watt, Parton distributions for the LHC, Eur. Phys. J. C 63, 189 (2009).

[77] ATLAS Collaboration, Luminosity determination in pp collisions at $\sqrt{s}=8 \mathrm{TeV}$ using the ATLAS detector at the LHC, Eur. Phys. J. C 76, 653 (2016).

[78] M. Cacciari, G. P. Salam, and G. Soyez, The anti- $k_{t}$ jet clustering algorithm, J. High Energy Phys. 04 (2008) 063.

[79] M. Cacciari, G. P. Salam, and G. Soyez, FastJet user manual, Eur. Phys. J. C 72, 1896 (2012).

[80] ATLAS Collaboration, Report No. ATL-PHYS-PUB2015-036, 2015, https://cds.cern.ch/record/2044564.

[81] M. Cacciari and G. P. Salam, Pileup subtraction using jet areas, Phys. Lett. B 659, 119 (2008).

[82] ATLAS Collaboration, Performance of pile-up mitigation techniques for jets in $p p$ collisions at $\sqrt{s}=8 \mathrm{TeV}$ using the ATLAS detector, Eur. Phys. J. C 76, 581 (2016).

[83] ATLAS Collaboration, Report No. ATL-PHYS-PUB2015-015, 2015, https://cds.cern.ch/record/2037613.

[84] ATLAS Collaboration, Jet energy scale measurements and their systematic uncertainties in proton-proton collisions at $\sqrt{s}=13 \mathrm{TeV}$ with the ATLAS detector, Phys. Rev. D 96, 072002 (2017).

[85] ATLAS Collaboration, Report No. ATLAS-CONF-2014018, 2014, https://cds.cern.ch/record/1700870.

[86] ATLAS Collaboration, Report No. ATL-PHYS-PUB2015-022, 2015, https://cds.cern.ch/record/2037697.

[87] ATLAS Collaboration, Report No. ATL-PHYS-PUB2015-039, 2015, https://cds.cern.ch/record/2047871.

[88] ATLAS Collaboration, Report No. ATL-PHYS-PUB2016-012, 2016, https://cds.cern.ch/record/2160731. 
[89] ATLAS Collaboration, Report No. ATLAS-CONF-2016024, 2016, https://cds.cern.ch/record/2157687.

[90] ATLAS Collaboration, Muon reconstruction performance of the ATLAS detector in proton-proton collision data at $\sqrt{s}=13 \mathrm{TeV}$, Eur. Phys. J. C 76, 292 (2016).

[91] ATLAS Collaboration, Report No. ATLAS-CONF-2015029, 2015, https://cds.cern.ch/record/2037702.

[92] ATLAS Collaboration, Report No. ATL-PHYS-PUB2015-023, 2015, https://cds.cern.ch/record/2037700.

[93] ATLAS Collaboration, Report No. ATL-PHYS-PUB2015-027, 2015, https://cds.cern.ch/record/2037904.

[94] C. Chen, New approach to identifying boosted hadronically decaying particles using jet substracture in its centerof-mass frame, Phys. Rev. D 85, 034007 (2012).

[95] S. Jadach, J. H. Kuhn, and Z. Was, TAUOLA: A library of Monte Carlo programs to simulate decays of polarized tau leptons, Comput. Phys. Commun. 64, 275 (1991).
[96] M. Bahr et al., Herwig++ Physics and Manual, Eur. Phys. J. C 58, 639 (2008).

[97] ATLAS Collaboration, Measurement of $W^{ \pm}$and Z-boson production cross sections in $p p$ collisions at $\sqrt{s}=13 \mathrm{TeV}$ with the ATLAS detector, Phys. Lett. B 759, 601 (2016).

[98] G. Cowan, K. Cranmer, E. Gross, and O. Vitells, Asymptotic formulae for likelihood-based tests of new physics, Eur. Phys. J. C 71, 1554 (2011).

[99] M. Baak, G. J. Besjes, D. Côté, A. Koutsman, J. Lorenz, and D. Short, HistFitter software framework for statistical data analysis, Eur. Phys. J. C 75, 153 (2015).

[100] A. L. Read, Presentation of search results: The CL(s) technique, J. Phys. G 28, 2693 (2002).

[101] ATLAS Collaboration, Report No. ATL-GEN-PUB-2016002, https://cds.cern.ch/record/2202407.

M. Aaboud, ${ }^{137 d}$ G. Aad ${ }^{88}$ B. Abbott, ${ }^{115}$ O. Abdinov, ${ }^{12, a}$ B. Abeloos, ${ }^{119}$ S. H. Abidi, ${ }^{161}$ O. S. AbouZeid, ${ }^{139}$ N. L. Abraham, ${ }^{151}$ H. Abramowicz, ${ }^{155}$ H. Abreu, ${ }^{154}$ R. Abreu ${ }^{118}$ Y. Abulaiti, ${ }^{148 a, 148 b}$ B. S. Acharya, ${ }^{167 a, 167 b, b}$ S. Adachi, ${ }^{157}$ L. Adamczyk, ${ }^{41 a}$ J. Adelman, ${ }^{110}$ M. Adersberger, ${ }^{102}$ T. Adye, ${ }^{133}$ A. A. Affolder, ${ }^{139}$ T. Agatonovic-Jovin, ${ }^{14}$ C. Agheorghiesei, ${ }^{28 c}$

J. A. Aguilar-Saavedra, ${ }^{128 a, 128 f}$ S. P. Ahlen, ${ }^{24}$ F. Ahmadov, ${ }^{68, c}$ G. Aielli, ${ }^{135 a, 135 b}$ S. Akatsuka, ${ }^{71}$ H. Akerstedt, ${ }^{148 a, 148 b}$ T. P. A. Åkesson, ${ }^{84}$ E. Akilli, ${ }^{52}$ A. V. Akimov, ${ }^{98}$ G. L. Alberghi, ${ }^{22 a, 22 b}$ J. Albert, ${ }^{172}$ P. Albicocco,${ }^{50}$ M. J. Alconada Verzini, ${ }^{74}$ S. C. Alderweireldt, ${ }^{108}$ M. Aleksa, ${ }^{32}$ I. N. Aleksandrov, ${ }^{68}$ C. Alexa, ${ }^{28 b}$ G. Alexander, ${ }^{155}$ T. Alexopoulos, ${ }^{10}$ M. Alhroob, ${ }^{115}$ B. Ali ${ }^{130}$ M. Aliev, ${ }^{76 a, 76 b}$ G. Alimonti, ${ }^{94 a}$ J. Alison, ${ }^{33}$ S. P. Alkire, ${ }^{38}$ B. M. M. Allbrooke, ${ }^{151}$ B. W. Allen, ${ }^{118}$ P. P. Allport, ${ }^{19}$ A. Aloisio, ${ }^{106 a, 106 b}$ A. Alonso, ${ }^{39}$ F. Alonso,${ }^{74}$ C. Alpigiani ${ }^{140}$ A. A. Alshehri, ${ }^{56}$ M. I. Alstaty ${ }^{88}$ B. Alvarez Gonzalez ${ }^{32}$ D. Álvarez Piqueras, ${ }^{170}$ M. G. Alviggi, ${ }^{106 a, 106 b}$ B. T. Amadio, ${ }^{16}$ Y. Amaral Coutinho, ${ }^{26 \mathrm{a}}$ C. Amelung, ${ }^{25}$ D. Amidei, ${ }^{92}$ S. P. Amor Dos Santos, ${ }^{128 a, 128 c}$ A. Amorim, ${ }^{128 a, 128 b}$ S. Amoroso, ${ }^{32}$ G. Amundsen, ${ }^{25}$ C. Anastopoulos, ${ }^{141}$ L. S. Ancu, ${ }^{52}$ N. Andari, ${ }^{19}$ T. Andeen, ${ }^{11}$ C. F. Anders, ${ }^{60 \mathrm{~b}}$ J. K. Anders, ${ }^{77}$ K. J. Anderson, ${ }^{33}$ A. Andreazza,${ }^{94 a, 94 b}$ V. Andrei, ${ }^{60 a}$ S. Angelidakis, ${ }^{9}$ I. Angelozzi, ${ }^{109}$ A. Angerami, ${ }^{38}$ A. V. Anisenkov, ${ }^{11, d}$ N. Anjos, ${ }^{13}$ A. Annovi, ${ }^{126 a, 126 b}$ C. Antel, ${ }^{60 a}$ M. Antonelli, ${ }^{50}$ A. Antonov, ${ }^{100, a}$ D. J. Antrim, ${ }^{166}$ F. Anulli, ${ }^{134 a}$ M. Aoki, ${ }^{69}$ L. Aperio Bella, ${ }^{32}$ G. Arabidze, ${ }^{93}$ Y. Arai,${ }^{69}$ J. P. Araque, ${ }^{128 a}$ V. Araujo Ferraz, ${ }^{26 a}$ A. T. H. Arce, ${ }^{48}$ R. E. Ardell, ${ }^{80}$ F. A. Arduh, ${ }^{74}$ J-F. Arguin, ${ }^{97}$ S. Argyropoulos,${ }^{66}$ M. Arik, ${ }^{20 a}$ A. J. Armbruster, ${ }^{32}$ L. J. Armitage, ${ }^{79}$ O. Arnaez, ${ }^{161}$ H. Arnold,${ }^{51}$ M. Arratia, ${ }^{30}$ O. Arslan, ${ }^{23}$ A. Artamonov, ${ }^{99}$ G. Artoni, ${ }^{122}$ S. Artz, ${ }^{86}$ S. Asai, ${ }^{157}$ N. Asbah,${ }^{45}$ A. Ashkenazi, ${ }^{155}$ L. Asquith, ${ }^{151}$ K. Assamagan, ${ }^{27}$ R. Astalos, ${ }^{146 a}$ M. Atkinson, ${ }^{169}$ N. B. Atlay, ${ }^{143}$ K. Augsten, ${ }^{130}$ G. Avolio, ${ }^{32}$ B. Axen, ${ }^{16}$ M. K. Ayoub, ${ }^{119}$ G. Azuelos, ${ }^{97, e}$ A. E. Baas, ${ }^{60 a}$ M. J. Baca, ${ }^{19}$ H. Bachacou, ${ }^{138}$ K. Bachas, ${ }^{76 a, 76 b}$ M. Backes, ${ }^{122}$ M. Backhaus, ${ }^{32}$ P. Bagnaia, ${ }^{134 a, 134 b}$ M. Bahmani, ${ }^{42}$ H. Bahrasemani, ${ }^{144}$ J. T. Baines, ${ }^{133}$ M. Bajic,${ }^{39}$ O. K. Baker, ${ }^{179}$ E. M. Baldin, ${ }^{11, d}$ P. Balek, ${ }^{175}$ F. Balli, ${ }^{138}$ W. K. Balunas, ${ }^{124}$ E. Banas, ${ }^{42}$ A. Bandyopadhyay, ${ }^{23}$ Sw. Banerjee, ${ }^{176, f}$ A. A. E. Bannoura, ${ }^{178}$ L. Barak,${ }^{32}$ E. L. Barberio, ${ }^{91}$ D. Barberis, ${ }^{53 a, 53 b}$ M. Barbero, ${ }^{88}$ T. Barillari, ${ }^{103}$ M-S Barisits, ${ }^{32}$ J. T. Barkeloo, ${ }^{118}$ T. Barklow, ${ }^{145}$ N. Barlow, ${ }^{30}$ S. L. Barnes, ${ }^{36 c}$ B. M. Barnett, ${ }^{133}$ R. M. Barnett, ${ }^{16}$ Z. Barnovska-Blenessy, ${ }^{36 a}$ A. Baroncelli, ${ }^{136 a}$ G. Barone,${ }^{25}$ A. J. Barr, ${ }^{122}$ L. Barranco Navarro, ${ }^{170}$ F. Barreiro, ${ }^{85}$ J. Barreiro Guimarães da Costa, ${ }^{35 a}$ R. Bartoldus, ${ }^{145}$ A. E. Barton, ${ }^{75}$ P. Bartos, ${ }^{146 a}$ A. Basalaev, ${ }^{125}$ A. Bassalat, ${ }^{119, g}$ R. L. Bates, ${ }^{56}$ S. J. Batista, ${ }^{161}$ J. R. Batley, ${ }^{30}$ M. Battaglia, ${ }^{139}$ M. Bauce, ${ }^{134 a, 134 b}$ F. Bauer, ${ }^{138}$ H. S. Bawa, ${ }^{145, h}$ J. B. Beacham, ${ }^{113}$ M. D. Beattie, ${ }^{75}$ T. Beau, ${ }^{83}$ P. H. Beauchemin, ${ }^{165}$ P. Bechtle ${ }^{23}$ H. P. Beck, ${ }^{18, i}$ H. C. Beck, ${ }^{57}$ K. Becker, ${ }^{122}$ M. Becker ${ }^{86}$ M. Beckingham, ${ }^{173}$ C. Becot,${ }^{112}$ A. J. Beddall, ${ }^{20 \mathrm{e}}$ A. Beddall, ${ }^{20 \mathrm{~b}}$ V. A. Bednyakov,${ }^{68}$ M. Bedognetti, ${ }^{109}$ C. P. Bee, ${ }^{150}$ T. A. Beermann, ${ }^{32}$ M. Begalli, ${ }^{26 a}$ M. Begel, ${ }^{27}$ J. K. Behr, ${ }^{45}$ A. S. Bell, ${ }^{81}$ G. Bella, ${ }^{155}$ L. Bellagamba, ${ }^{22 a}$ A. Bellerive,${ }^{31}$ M. Bellomo, ${ }^{154}$ K. Belotskiy, ${ }^{100}$ O. Beltramello, ${ }^{32}$ N. L. Belyaev, ${ }^{100}$ O. Benary, ${ }^{155, a}$ D. Benchekroun, ${ }^{137 a}$ M. Bender, ${ }^{102}$ K. Bendtz, ${ }^{148 a, 148 b}$ N. Benekos, ${ }^{10}$ Y. Benhammou, ${ }^{155}$ E. Benhar Noccioli, ${ }^{179}$ J. Benitez, ${ }^{66}$ D. P. Benjamin, ${ }^{48}$ M. Benoit, ${ }^{52}$ J. R. Bensinger, ${ }^{25}$ S. Bentvelsen, ${ }^{109}$ L. Beresford, ${ }^{122}$ M. Beretta, ${ }^{50}$ D. Berge, ${ }^{109}$ E. Bergeaas Kuutmann, ${ }^{168}$ N. Berger, ${ }^{5}$ J. Beringer, ${ }^{16}$ S. Berlendis, ${ }^{58}$ N. R. Bernard ${ }^{89}$ G. Bernardi, ${ }^{83}$ C. Bernius, ${ }^{145}$ F. U. Bernlochner, ${ }^{23}$ T. Berry,${ }^{80}$ P. Berta, ${ }^{131}$ C. Bertella, ${ }^{35 \mathrm{a}}$ G. Bertoli, ${ }^{148 \mathrm{a}, 148 \mathrm{~b}}$ F. Bertolucci, ${ }^{126 \mathrm{a}, 126 \mathrm{~b}}$ I. A. Bertram, ${ }^{75}$ C. Bertsche, ${ }^{45}$ D. Bertsche, ${ }^{115}$ 
G. J. Besjes, ${ }^{39}$ O. Bessidskaia Bylund, ${ }^{148 a, 148 b}$ M. Bessner, ${ }^{45}$ N. Besson, ${ }^{138}$ C. Betancourt, ${ }^{51}$ A. Bethani, ${ }^{87}$ S. Bethke, ${ }^{103}$ A. J. Bevan, ${ }^{79}$ J. Beyer, ${ }^{103}$ R. M. Bianchi, ${ }^{127}$ O. Biebel,${ }^{102}$ D. Biedermann, ${ }^{17}$ R. Bielski,${ }^{87}$ K. Bierwagen, ${ }^{86}$ N. V. Biesuz, ${ }^{126 a, 126 b}$ M. Biglietti, ${ }^{136 a}$ T. R. V. Billoud,${ }^{97}$ H. Bilokon, ${ }^{50}$ M. Bindi, ${ }^{57}$ A. Bingul, ${ }^{20 b}$ C. Bini, ${ }^{134 a, 134 b}$ S. Biondi, ${ }^{22 a, 22 b}$ T. Bisanz ${ }^{57}$ C. Bittrich, ${ }^{47}$ D. M. Bjergaard, ${ }^{48}$ C. W. Black, ${ }^{152}$ J. E. Black, ${ }^{145}$ K. M. Black, ${ }^{24}$ R. E. Blair, ${ }^{6}$ T. Blazek, ${ }^{146 a}$ I. Bloch, ${ }^{45}$ C. Blocker, ${ }^{25}$ A. Blue ${ }^{56}$ W. Blum,${ }^{86, a}$ U. Blumenschein ${ }^{79}$ S. Blunier, ${ }^{34 a}$ G. J. Bobbink, ${ }^{109}$ V. S. Bobrovnikov, ${ }^{11, d}$ S. S. Bocchetta, ${ }^{84}$ A. Bocci ${ }^{48}$ C. Bock, ${ }^{102}$ M. Boehler, ${ }^{51}$ D. Boerner, ${ }^{178}$ D. Bogavac, ${ }^{102}$ A. G. Bogdanchikov, ${ }^{111}$ C. Bohm, ${ }^{148 a}$ V. Boisvert ${ }^{80}$ P. Bokan, ${ }^{168, j}$ T. Bold, ${ }^{41 a}$ A. S. Boldyrev, ${ }^{101}$ A. E. Bolz, ${ }^{60 b}$ M. Bomben, ${ }^{83}$ M. Bona, ${ }^{79}$ M. Boonekamp, ${ }^{138}$ A. Borisov, ${ }^{132}$ G. Borissov, ${ }^{75} \mathrm{~J}$. Bortfeldt, ${ }^{32}$ D. Bortoletto, ${ }^{122}$ V. Bortolotto, ${ }^{62 a}$ D. Boscherini, ${ }^{22 a}$ M. Bosman, ${ }^{13}$ J. D. Bossio Sola, ${ }^{29}$ J. Boudreau, ${ }^{127}$ J. Bouffard, ${ }^{2}$ E. V. Bouhova-Thacker, ${ }^{75}$

D. Boumediene, ${ }^{37}$ C. Bourdarios, ${ }^{119}$ S. K. Boutle,${ }^{56}$ A. Boveia, ${ }^{113}$ J. Boyd ${ }^{32}$ I. R. Boyko, ${ }^{68}$ J. Bracinik, ${ }^{19}$ A. Brandt, ${ }^{8}$ G. Brandt, ${ }^{57}$ O. Brandt, ${ }^{60 a}$ U. Bratzler,${ }^{158}$ B. Brau, ${ }^{89}$ J. E. Brau, ${ }^{118}$ W. D. Breaden Madden, ${ }^{56}$ K. Brendlinger, ${ }^{45}$ A. J. Brennan, ${ }^{91}$ L. Brenner, ${ }^{109}$ R. Brenner, ${ }^{168}$ S. Bressler, ${ }^{175}$ D. L. Briglin, ${ }^{19}$ T. M. Bristow, ${ }^{49}$ D. Britton, ${ }^{56}$ D. Britzger, ${ }^{45}$ F. M. Brochu, ${ }^{30}$ I. Brock, ${ }^{23}$ R. Brock,,${ }^{93}$ G. Brooijmans, ${ }^{38}$ T. Brooks, ${ }^{80}$ W. K. Brooks, ${ }^{34 b}$ J. Brosamer, ${ }^{16}$ E. Brost, ${ }^{110}$ J. H Broughton, ${ }^{19}$ P. A. Bruckman de Renstrom, ${ }^{42}$ D. Bruncko, ${ }^{146 \mathrm{~b}}$ A. Bruni, ${ }^{22 \mathrm{a}}$ G. Bruni, ${ }^{22 \mathrm{a}}$ L. S. Bruni, ${ }^{109}$ BH Brunt,${ }^{30}$ M. Bruschi, ${ }^{22 a}$ N. Bruscino, ${ }^{23}$ P. Bryant,${ }^{33}$ L. Bryngemark, ${ }^{45}$ T. Buanes,${ }^{15}$ Q. Buat, ${ }^{144}$ P. Buchholz, ${ }^{143}$ A. G. Buckley, ${ }^{56}$ I. A. Budagov, ${ }^{68}$ F. Buehrer, ${ }^{51}$ M. K. Bugge, ${ }^{121}$ O. Bulekov ${ }^{100}$ D. Bullock, ${ }^{8}$ T. J. Burch, ${ }^{110}$ S. Burdin, ${ }^{77}$ C. D. Burgard, ${ }^{51}$ A. M. Burger, ${ }^{5}$ B. Burghgrave, ${ }^{110}$ K. Burka, ${ }^{42}$ S. Burke, ${ }^{133}$ I. Burmeister,${ }^{46}$ J. T. P. Burr, ${ }^{122}$ E. Busato, ${ }^{37}$ D. Büscher,${ }^{51}$ V. Büscher, ${ }^{86}$ P. Bussey, ${ }^{56}$ J. M. Butler, ${ }^{24}$ C. M. Buttar ${ }^{56}$ J. M. Butterworth,${ }^{81}$ P. Butti, ${ }^{32}$ W. Buttinger, ${ }^{27}$ A. Buzatu, ${ }^{35 c}$ A. R. Buzykaev, ${ }^{11, d}$ S. Cabrera Urbán, ${ }^{170}$ D. Caforio, ${ }^{130}$ V. M. Cairo, ${ }^{40 a, 40 b}$ O. Cakir, ${ }^{4 a}$ N. Calace, ${ }^{52}$ P. Calafiura, ${ }^{16}$ A. Calandri, ${ }^{88}$ G. Calderini, ${ }^{83}$ P. Calfayan, ${ }^{64}$ G. Callea, ${ }^{40 a, 40 b}$ L. P. Caloba, ${ }^{26 a}$ S. Calvente Lopez, ${ }^{85}$ D. Calvet, ${ }^{37}$ S. Calvet, ${ }^{37}$ T. P. Calvet, ${ }^{88}$ R. Camacho Toro, ${ }^{33}$ S. Camarda, ${ }^{32}$ P. Camarri, ${ }^{135 a, 135 b}$ D. Cameron, ${ }^{121}$ R. Caminal Armadans, ${ }^{169}$ C. Camincher ${ }^{58}$ S. Campana, ${ }^{32}$ M. Campanelli, ${ }^{81}$ A. Camplani, ${ }^{94 a, 94 b}$ A. Campoverde,${ }^{143}$ V. Canale,${ }^{106 a, 106 b}$ M. Cano Bret, ${ }^{36 c}$ J. Cantero, ${ }^{116}$ T. Cao, ${ }^{155}$ M. D. M. Capeans Garrido, ${ }^{32}$ I. Caprini, ${ }^{28 b}$ M. Caprini, ${ }^{28 b}$ M. Capua, ${ }^{40 a, 40 b}$ R. M. Carbone, ${ }^{38}$ R. Cardarelli, ${ }^{135 a}$ F. Cardillo, ${ }^{51}$ I. Carli, ${ }^{131}$ T. Carli, ${ }^{32}$ G. Carlino, ${ }^{106 a}$ B. T. Carlson, ${ }^{127}$ L. Carminati, ${ }^{94 a, 94 b}$

R. M. D. Carney, ${ }^{148 a, 148 b}$ S. Caron, ${ }^{108}$ E. Carquin, ${ }^{34 b}$ S. Carrá, ${ }^{94 a, 94 b}$ G. D. Carrillo-Montoya, ${ }^{32}$ J. Carvalho, ${ }^{128 a, 128 c}$ D. Casadei, ${ }^{19}$ M. P. Casado, ${ }^{13, k}$ M. Casolino, ${ }^{13}$ D. W. Casper ${ }^{166}$ R. Castelijn,${ }^{109}$ V. Castillo Gimenez, ${ }^{170}$ N. F. Castro, ${ }^{128 a, 1}$ A. Catinaccio, ${ }^{32}$ J. R. Catmore, ${ }^{121}$ A. Cattai, ${ }^{32}$ J. Caudron, ${ }^{23}$ V. Cavaliere, ${ }^{169}$ E. Cavallaro, ${ }^{13}$ D. Cavalli, ${ }^{94 a}$

M. Cavalli-Sforza ${ }^{13}$ V. Cavasinni, ${ }^{126 a, 126 b}$ E. Celebi, ${ }^{20 \mathrm{~d}}$ F. Ceradini, ${ }^{136 a, 136 \mathrm{~b}}$ L. Cerda Alberich, ${ }^{170}$ A. S. Cerqueira, ${ }^{26 \mathrm{~b}}$ A. Cerri, ${ }^{151}$ L. Cerrito, ${ }^{135 a, 135 b}$ F. Cerutti, ${ }^{16}$ A. Cervelli, ${ }^{18}$ S. A. Cetin, ${ }^{20 d}$ A. Chafaq, ${ }^{137 a}$ D. Chakraborty, ${ }^{110}$ S. K. Chan,${ }^{59}$ W. S. Chan, ${ }^{109}$ Y. L. Chan, ${ }^{62 a}$ P. Chang, ${ }^{169}$ J. D. Chapman, ${ }^{30}$ D. G. Charlton, ${ }^{19}$ C. C. Chau, ${ }^{161}$ C. A. Chavez Barajas, ${ }^{151}$

S. Che, ${ }^{113}$ S. Cheatham, ${ }^{167 a, 167 c}$ A. Chegwidden, ${ }^{93}$ S. Chekanov, ${ }^{6}$ S. V. Chekulaev, ${ }^{163 a}$ G. A. Chelkov, ${ }^{68, m}$

M. A. Chelstowska, ${ }^{32}$ C. Chen, ${ }^{67}$ H. Chen, ${ }^{27}$ J. Chen, ${ }^{36 a}$ S. Chen, ${ }^{35 b}$ S. Chen,${ }^{157}$ X. Chen, ${ }^{35 c, n}$ Y. Chen,${ }^{70}$ H. C. Cheng, ${ }^{92}$ H. J. Cheng, ${ }^{35 a, 35 d}$ A. Cheplakov, ${ }^{68}$ E. Cheremushkina, ${ }^{132}$ R. Cherkaoui El Moursli, ${ }^{137 e}$ E. Cheu, ${ }^{7}$ K. Cheung, ${ }^{63}$

L. Chevalier, ${ }^{138}$ V. Chiarella, ${ }^{50}$ G. Chiarelli, ${ }^{126 a, 126 b}$ G. Chiodini, ${ }^{76 a}$ A. S. Chisholm, ${ }^{32}$ A. Chitan, ${ }^{28 b}$ Y. H. Chiu, ${ }^{172}$ M. V. Chizhov, ${ }^{68}$ K. Choi,${ }^{64}$ A. R. Chomont, ${ }^{37}$ S. Chouridou, ${ }^{156}$ V. Christodoulou, ${ }^{81}$ D. Chromek-Burckhart, ${ }^{32}$ M. C. Chu, ${ }^{62 a}$ J. Chudoba, ${ }^{129}$ A. J. Chuinard, ${ }^{90}$ J. J. Chwastowski, ${ }^{42}$ L. Chytka, ${ }^{117}$ A. K. Ciftci, ${ }^{4 a}$ D. Cinca, ${ }^{46}$ V. Cindro, ${ }^{78}$ I. A. Cioara, ${ }^{23}$ C. Ciocca, ${ }^{22 a, 22 b}$ A. Ciocio, ${ }^{16}$ F. Cirotto, ${ }^{106 a, 106 b}$ Z. H. Citron, ${ }^{175}$ M. Citterio, ${ }^{94 a}$ M. Ciubancan, ${ }^{28 b}$ A. Clark,${ }^{52}$ B. L. Clark, ${ }^{59}$ M. R. Clark, ${ }^{38}$ P. J. Clark, ${ }^{49}$ R. N. Clarke, ${ }^{16}$ C. Clement, ${ }^{148 a, 148 b}$ Y. Coadou, ${ }^{88}$ M. Cobal,${ }^{167 a, 167 \mathrm{c}}$ A. Coccaro, ${ }^{52}$ J. Cochran, ${ }^{67}$

L. Colasurdo, ${ }^{108}$ B. Cole, ${ }^{38}$ A. P. Colijn, ${ }^{109}$ J. Collot, ${ }^{58}$ T. Colombo, ${ }^{166}$ P. Conde Muiño, ${ }^{128 a, 128 b}$ E. Coniavitis, ${ }^{51}$ S. H. Connell, ${ }^{147 b}$ I. A. Connelly, ${ }^{87}$ S. Constantinescu, ${ }^{28 b}$ G. Conti, ${ }^{32}$ F. Conventi, ${ }^{106 a, 0}$ M. Cooke, ${ }^{16}$ A. M. Cooper-Sarkar, ${ }^{122}$ F. Cormier, ${ }^{171}$ K. J. R. Cormier, ${ }^{161}$ M. Corradi, ${ }^{134 a, 134 b}$ F. Corriveau, ${ }^{90, p}$ A. Cortes-Gonzalez, ${ }^{32}$ G. Cortiana, ${ }^{103}$ G. Costa, ${ }^{94 a}$ M. J. Costa, ${ }^{170}$ D. Costanzo, ${ }^{141}$ G. Cottin, ${ }^{30}$ G. Cowan, ${ }^{80}$ B. E. Cox,${ }^{87}$ K. Cranmer, ${ }^{12}$ S. J. Crawley, ${ }^{56}$ R. A. Creager, ${ }^{124}$ G. Cree, ${ }^{31}$ S. Crépé-Renaudin, ${ }^{58}$ F. Crescioli, ${ }^{83}$ W. A. Cribbs, ${ }^{148 a, 148 b}$ M. Cristinziani, ${ }^{23}$ V. Croft,${ }^{108}$ G. Crosetti, ${ }^{40 a, 40 b}$ A. Cueto, ${ }^{85}$ T. Cuhadar Donszelmann, ${ }^{141}$ A. R. Cukierman, ${ }^{145}$ J. Cummings,${ }^{179}$ M. Curatolo, ${ }^{50}$ J. Cúth ${ }^{86}$ P. Czodrowski, ${ }^{32}$ G. D'amen, ${ }^{22 a, 22 b}$ S. D'Auria, ${ }^{56}$ L. D'eramo, ${ }^{83}$ M. D’Onofrio, ${ }^{77}$ M. J. Da Cunha Sargedas De Sousa, ${ }^{128 a, 128 b}$ C. Da Via, ${ }^{87}$ W. Dabrowski, ${ }^{41 \mathrm{a}}$ T. Dado, ${ }^{146 a}$ T. Dai, ${ }^{92}$ O. Dale, ${ }^{15}$ F. Dallaire,${ }^{97}$ C. Dallapiccola ${ }^{89}$ M. Dam, ${ }^{39}$ J. R. Dandoy,${ }^{124}$ M. F. Daneri, ${ }^{29}$ N. P. Dang, ${ }^{176}$ A. C. Daniells, ${ }^{19}$ N. S. Dann, ${ }^{87}$ M. Danninger, ${ }^{171}$ M. Dano Hoffmann, ${ }^{138}$ V. Dao, ${ }^{150}$ G. Darbo, ${ }^{53 a}$ S. Darmora, ${ }^{8}$ J. Dassoulas, ${ }^{3}$ A. Dattagupta, ${ }^{118}$ T. Daubney,${ }^{45}$ W. Davey, ${ }^{23}$ C. David, ${ }^{45}$ T. Davidek,${ }^{131}$ D. R. Davis, ${ }^{48}$ P. Davison, ${ }^{81}$ E. Dawe,${ }^{91}$ I. Dawson,${ }^{141}$ K. De,${ }^{8}$ R. de Asmundis, ${ }^{106 a}$ A. De Benedetti, ${ }^{115}$ S. De Castro, ${ }^{22 a, 22 b}$ 
S. De Cecco, ${ }^{83}$ N. De Groot, ${ }^{108}$ P. de Jong, ${ }^{109}$ H. De la Torre, ${ }^{93}$ F. De Lorenzi, ${ }^{67}$ A. De Maria, ${ }^{57}$ D. De Pedis, ${ }^{134 a}$ A. De Salvo, ${ }^{134 a}$ U. De Sanctis, ${ }^{135 a, 135 b}$ A. De Santo, ${ }^{151}$ K. De Vasconcelos Corga, ${ }^{88}$ J. B. De Vivie De Regie, ${ }^{119}$ W. J. Dearnaley, ${ }^{75}$ R. Debbe,${ }^{27}$ C. Debenedetti, ${ }^{139}$ D. V. Dedovich, ${ }^{68}$ N. Dehghanian, ${ }^{3}$ I. Deigaard,${ }^{109}$ M. Del Gaudio, ${ }^{40 a, 40 b}$ J. Del Peso ${ }^{85}$ D. Delgove, ${ }^{119}$ F. Deliot, ${ }^{138}$ C. M. Delitzsch, ${ }^{7}$ A. Dell'Acqua, ${ }^{32}$ L. Dell'Asta, ${ }^{24}$ M. Dell'Orso, ${ }^{126 a, 126 b}$ M. Della Pietra, ${ }^{106 a, 106 b}$ D. della Volpe,${ }^{52}$ M. Delmastro, ${ }^{5}$ C. Delporte, ${ }^{119}$ P. A. Delsart, ${ }^{58}$ D. A. DeMarco, ${ }^{161}$ S. Demers, ${ }^{179}$ M. Demichev ${ }^{68}$ A. Demilly, ${ }^{83}$ S. P. Denisov ${ }^{132}$ D. Denysiuk, ${ }^{138}$ D. Derendarz,${ }^{42}$ J. E. Derkaoui, ${ }^{137 \mathrm{~d}}$ F. Derue,${ }^{83}$ P. Dervan,${ }^{77}$ K. Desch, ${ }^{23}$ C. Deterre, ${ }^{45}$ K. Dette,${ }^{46}$ M. R. Devesa, ${ }^{29}$ P. O. Deviveiros, ${ }^{32}$ A. Dewhurst, ${ }^{133}$ S. Dhaliwal, ${ }^{25}$ F. A. Di Bello, ${ }^{52}$ A. Di Ciaccio, ${ }^{135 a, 135 b}$ L. Di Ciaccio, ${ }^{5}$ W. K. Di Clemente, ${ }^{124}$ C. Di Donato, ${ }^{106 a, 106 b}$ A. Di Girolamo, ${ }^{32}$ B. Di Girolamo, ${ }^{32}$ B. Di Micco, ${ }^{136 a, 136 b}$ R. Di Nardo, ${ }^{32}$ K. F. Di Petrillo, ${ }^{59}$ A. Di Simone, ${ }^{51}$ R. Di Sipio, ${ }^{161}$ D. Di Valentino, ${ }^{31}$ C. Diaconu, ${ }^{88}$ M. Diamond ${ }^{161}$ F. A. Dias, ${ }^{39}$ M. A. Diaz,${ }^{34 a}$ E. B. Diehl,${ }^{92}$ J. Dietrich, ${ }^{17}$ S. Díez Cornell, ${ }^{45}$ A. Dimitrievska, ${ }^{14}$ J. Dingfelder, ${ }^{23}$ P. Dita, ${ }^{28 b}$ S. Dita, ${ }^{28 b}$ F. Dittus, ${ }^{32}$ F. Djama, ${ }^{88}$ T. Djobava, ${ }^{54 b}$ J. I. Djuvsland, ${ }^{60 \mathrm{a}}$ M. A. B. do Vale, ${ }^{260}$ D. Dobos, ${ }^{32}$ M. Dobre,${ }^{28 b}$ C. Doglioni, ${ }^{84}$ J. Dolejsi, ${ }^{131}$ Z. Dolezal, ${ }^{131}$ M. Donadelli, ${ }^{26 \mathrm{~d}}$ S. Donati, ${ }^{126 a, 126 \mathrm{~b}}$ P. Dondero, ${ }^{123 a, 123 \mathrm{~b}}$ J. Donini, ${ }^{37}$ J. Dopke, ${ }^{133}$ A. Doria, ${ }^{106 a}$ M. T. Dova,${ }^{74}$ A. T. Doyle,${ }^{56}$ E. Drechsler, ${ }^{57}$ M. Dris,${ }^{10}$ Y. Du, ${ }^{36 b}$ J. Duarte-Campderros, ${ }^{155}$ A. Dubreuil,${ }^{52}$ E. Duchovni, ${ }^{175}$ G. Duckeck, ${ }^{102}$ A. Ducourthial, ${ }^{83}$ O. A. Ducu, ${ }^{97, q}$ D. Duda, ${ }^{109}$ A. Dudarev, ${ }^{32}$ A. Chr. Dudder, ${ }^{86}$ E. M. Duffield, ${ }^{16}$ L. Duflot, ${ }^{119}$ M. Dührssen, ${ }^{32}$ M. Dumancic, ${ }^{175}$ A. E. Dumitriu, ${ }^{28 b}$ A. K. Duncan, ${ }^{56}$ M. Dunford, ${ }^{60 a}$ H. Duran Yildiz,${ }^{4 a}$ M. Düren, ${ }^{55}$ A. Durglishvili, ${ }^{54 b}$ D. Duschinger,${ }^{47}$ B. Dutta, ${ }^{45}$ D. Duvnjak, ${ }^{1}$ M. Dyndal, ${ }^{45}$ B. S. Dziedzic, ${ }^{42}$ C. Eckardt, ${ }^{45}$ K. M. Ecker, ${ }^{103}$ R. C. Edgar, ${ }^{92}$ T. Eifert, ${ }^{32}$ G. Eigen, ${ }^{15}$ K. Einsweiler ${ }^{16}$ T. Ekelof, ${ }^{168}$ M. El Kacimi, ${ }^{137 \mathrm{c}}$ R. El Kosseifi, ${ }^{88}$ V. Ellajosyula, ${ }^{88}$ M. Ellert, ${ }^{168}$ S. Elles, ${ }^{5}$ F. Ellinghaus, ${ }^{178}$ A. A. Elliot,${ }^{172}$ N. Ellis, ${ }^{32}$ J. Elmsheuser,${ }^{27}$ M. Elsing, ${ }^{32}$ D. Emeliyanov, ${ }^{133}$ Y. Enari, ${ }^{157}$ O. C. Endner, ${ }^{86}$ J. S. Ennis, ${ }^{173}$ J. Erdmann, ${ }^{46}$ A. Ereditato, ${ }^{18}$ M. Ernst, ${ }^{27}$ S. Errede, ${ }^{169}$ M. Escalier, ${ }^{119}$ C. Escobar, ${ }^{170}$ B. Esposito, ${ }^{50}$ O. Estrada Pastor, ${ }^{170}$ A. I. Etienvre, ${ }^{138}$ E. Etzion, ${ }^{155}$ H. Evans, ${ }^{64}$ A. Ezhilov, ${ }^{125}$ M. Ezzi, ${ }^{137 e}$ F. Fabbri, ${ }^{22 a, 22 b}$ L. Fabbri, ${ }^{22 a, 22 b}$ V. Fabiani, ${ }^{108}$ G. Facini ${ }^{81}$ R. M. Fakhrutdinov, ${ }^{132}$ S. Falciano, ${ }^{134 a}$ R. J. Falla, ${ }^{81}$ J. Faltova, ${ }^{32}$ Y. Fang, ${ }^{35 a}$ M. Fanti, ${ }^{94 a, 94 b}$ A. Farbin, ${ }^{8}$ A. Farilla, ${ }^{136 a}$ C. Farina, ${ }^{127}$ E. M. Farina, ${ }^{123 a, 123 b}$ T. Farooque, ${ }^{93}$ S. Farrell, ${ }^{16}$ S. M. Farrington, ${ }^{173}$ P. Farthouat, ${ }^{32}$ F. Fassi, ${ }^{137 e}$ P. Fassnacht, ${ }^{32}$ D. Fassouliotis, ${ }^{9}$ M. Faucci Giannelli, ${ }^{80}$ A. Favareto, ${ }^{53 a, 53 b}$ W. J. Fawcett, ${ }^{122}$ L. Fayard, ${ }^{119}$ O. L. Fedin, ${ }^{125, r}$ W. Fedorko, ${ }^{171}$ S. Feigl, ${ }^{121}$ L. Feligioni, ${ }^{88}$ C. Feng, ${ }^{36 \mathrm{~b}}$ E. J. Feng, ${ }^{32}$ H. Feng, ${ }^{92}$ M. J. Fenton, ${ }^{56}$ A. B. Fenyuk, ${ }^{132}$ L. Feremenga, ${ }^{8}$ P. Fernandez Martinez, ${ }^{170}$ S. Fernandez Perez, ${ }^{13}$ J. Ferrando, ${ }^{45}$ A. Ferrari, ${ }^{168}$ P. Ferrari, ${ }^{109}$ R. Ferrari, ${ }^{123 a}$ D. E. Ferreira de Lima, ${ }^{60 b}$ A. Ferrer,${ }^{170}$ D. Ferrere,${ }^{52}$ C. Ferretti,${ }^{92}$ F. Fiedler, ${ }^{86}$ A. Filipčič,${ }^{78}$ M. Filipuzzi,${ }^{45}$ F. Filthaut, ${ }^{108}$ M. Fincke-Keeler, ${ }^{172}$ K. D. Finelli, ${ }^{152}$ M. C. N. Fiolhais, ${ }^{128 a, 128 c, s}$ L. Fiorini, ${ }^{170}$ A. Fischer, ${ }^{2}$ C. Fischer,${ }^{13}$ J. Fischer, ${ }^{178}$ W. C. Fisher, ${ }^{93}$ N. Flaschel, ${ }^{45}$ I. Fleck, ${ }^{143}$ P. Fleischmann, ${ }^{92}$ R. R. M. Fletcher, ${ }^{124}$ T. Flick, ${ }^{178}$ B. M. Flierl, ${ }^{102}$ L. R. Flores Castillo, ${ }^{62 a}$ M. J. Flowerdew, ${ }^{103}$ G. T. Forcolin, ${ }^{87}$ A. Formica, ${ }^{138}$ F. A. Förster, ${ }^{13}$ A. Forti, ${ }^{87}$ A. G. Foster, ${ }^{19}$ D. Fournier, ${ }^{119}$ H. Fox, ${ }^{75}$ S. Fracchia,${ }^{141}$ P. Francavilla, ${ }^{83}$ M. Franchini, ${ }^{22 a, 22 b}$ S. Franchino, ${ }^{60 a}$ D. Francis,${ }^{32}$ L. Franconi, ${ }^{121}$ M. Franklin, ${ }^{59}$ M. Frate, ${ }^{166}$ M. Fraternali, ${ }^{123 a, 123 b}$ D. Freeborn, ${ }^{81}$ S. M. Fressard-Batraneanu, ${ }^{32}$ B. Freund, ${ }^{97}$ D. Froidevaux, ${ }^{32}$ J. A. Frost, ${ }^{122}$ C. Fukunaga, ${ }^{158}$ T. Fusayasu, ${ }^{104}$ J. Fuster, ${ }^{170}$ C. Gabaldon, ${ }^{58}$ O. Gabizon, ${ }^{154}$ A. Gabrielli, ${ }^{22 a, 22 b}$ A. Gabrielli, ${ }^{16}$ G. P. Gach, ${ }^{41 \mathrm{a}}$ S. Gadatsch, ${ }^{32}$ S. Gadomski, ${ }^{80}$ G. Gagliardi, ${ }^{53 a, 53 b}$ L. G. Gagnon, ${ }^{97}$ C. Galea, ${ }^{108}$ B. Galhardo, ${ }^{128 a, 128 c}$ E. J. Gallas, ${ }^{122}$ B. J. Gallop, ${ }^{133}$ P. Gallus, ${ }^{130}$ G. Galster, ${ }^{39}$ K. K. Gan, ${ }^{113}$ S. Ganguly, ${ }^{37}$ Y. Gao, ${ }^{77}$ Y. S. Gao, ${ }^{145, h}$ F. M. Garay Walls, ${ }^{49}$ C. García, ${ }^{170}$ J. E. García Navarro, ${ }^{170}$ J. A. García Pascual, ${ }^{35 a}$ M. Garcia-Sciveres, ${ }^{16}$ R. W. Gardner, ${ }^{33}$ N. Garelli, ${ }^{145}$ V. Garonne, ${ }^{121}$ A. Gascon Bravo,${ }^{45}$ K. Gasnikova, ${ }^{45}$ C. Gatti, ${ }^{50}$ A. Gaudiello, ${ }^{53 a, 53 b}$ G. Gaudio, ${ }^{123 a}$ I. L. Gavrilenko, ${ }^{98}$ C. Gay, ${ }^{171}$ G. Gaycken, ${ }^{23}$ E. N. Gazis, ${ }^{10}$ C. N. P. Gee, ${ }^{133}$ J. Geisen, ${ }^{57}$ M. Geisen, ${ }^{86}$ M. P. Geisler, ${ }^{60 a}$ K. Gellerstedt, ${ }^{148 a, 148 b}$ C. Gemme, ${ }^{53 a}$ M. H. Genest, ${ }^{58}$ C. Geng, ${ }^{92}$ S. Gentile, ${ }^{134 a, 134 b}$ C. Gentsos,${ }^{156}$ S. George, ${ }^{80}$ D. Gerbaudo, ${ }^{13}$ A. Gershon, ${ }^{155}$ G. Geßner, ${ }^{46}$ S. Ghasemi, ${ }^{143}$ M. Ghneimat, ${ }^{23}$ B. Giacobbe, ${ }^{22 a}$ S. Giagu, ${ }^{134 a, 134 b}$ N. Giangiacomi, ${ }^{22 a, 22 b}$ P. Giannetti, ${ }^{126 a, 126 b}$ S. M. Gibson, ${ }^{80}$ M. Gignac, ${ }^{171}$ M. Gilchriese, ${ }^{16}$ D. Gillberg, ${ }^{31}$ G. Gilles, ${ }^{178}$ D. M. Gingrich, ${ }^{3, e}$ N. Giokaris, ${ }^{9, a}$ M. P. Giordani, ${ }^{167 a, 167 c}$ F. M. Giorgi, ${ }^{22 a}$ P. F. Giraud, ${ }^{138}$ P. Giromini, ${ }^{59}$ G. Giugliarelli, ${ }^{167 a, 167 \mathrm{c}}$ D. Giugni, ${ }^{94 \mathrm{a}}$ F. Giuli, ${ }^{122}$ C. Giuliani, ${ }^{103}$ M. Giulini, ${ }^{60 \mathrm{~b}}$ B. K. Gjelsten, ${ }^{121}$ S. Gkaitatzis, ${ }^{156}$ I. Gkialas, ${ }^{9, \mathrm{t}}$ E. L. Gkougkousis, ${ }^{139}$ P. Gkountoumis, ${ }^{10}$ L. K. Gladilin, ${ }^{101}$ C. Glasman, ${ }^{85}$ J. Glatzer, ${ }^{13}$ P. C. F. Glaysher, ${ }^{45}$ A. Glazov, ${ }^{45}$ M. Goblirsch-Kolb, ${ }^{25}$ J. Godlewski, ${ }^{42}$ S. Goldfarb, ${ }^{91}$ T. Golling, ${ }^{52}$ D. Golubkov, ${ }^{132}$ A. Gomes, ${ }^{128 a, 128 b, 128 d}$ R. Gonçalo, ${ }^{128 a}$ R. Goncalves Gama, ${ }^{26 a}$ J. Goncalves Pinto Firmino Da Costa, ${ }^{138}$ G. Gonella, ${ }^{51}$ L. Gonella, ${ }^{19}$ A. Gongadze, ${ }^{68}$

S. González de la Hoz, ${ }^{170}$ S. Gonzalez-Sevilla, ${ }^{52}$ L. Goossens, ${ }^{32}$ P. A. Gorbounov, ${ }^{99}$ H. A. Gordon, ${ }^{27}$ I. Gorelov, ${ }^{107}$ B. Gorini, ${ }^{32}$ E. Gorini, ${ }^{76 a, 76 b}$ A. Gorišek,${ }^{78}$ A. T. Goshaw, ${ }^{48}$ C. Gössling, ${ }^{46}$ M. I. Gostkin, ${ }^{68}$ C. A. Gottardo,${ }^{23}$ C. R. Goudet, ${ }^{119}$ D. Goujdami, ${ }^{137 \mathrm{c}}$ A. G. Goussiou, ${ }^{140}$ N. Govender, ${ }^{147 \mathrm{~b}, \mathrm{u}}$ E. Gozani, ${ }^{154}$ L. Graber, ${ }^{57}$ I. Grabowska-Bold, ${ }^{41 \mathrm{a}}$ P. O. J. Gradin, ${ }^{168}$ 
J. Gramling, ${ }^{166}$ E. Gramstad, ${ }^{121}$ S. Grancagnolo, ${ }^{17}$ V. Gratchev, ${ }^{125}$ P. M. Gravila, ${ }^{28 f}$ C. Gray, ${ }^{56}$ H. M. Gray, ${ }^{16}$ Z. D. Greenwood, ${ }^{82, v}$ C. Grefe, ${ }^{23}$ K. Gregersen ${ }^{81}$ I. M. Gregor, ${ }^{45}$ P. Grenier ${ }^{145}$ K. Grevtsov, ${ }^{5}$ J. Griffiths ${ }^{8}$ A. A. Grillo, ${ }^{139}$ K. Grimm, ${ }^{75}$ S. Grinstein, ${ }^{13, w}$ Ph. Gris,${ }^{37}$ J.-F. Grivaz, ${ }^{119}$ S. Groh, ${ }^{86}$ E. Gross, ${ }^{175}$ J. Grosse-Knetter, ${ }^{57}$ G. C. Grossi, ${ }^{82}$ Z. J. Grout, ${ }^{81}$ A. Grummer, ${ }^{107}$ L. Guan, ${ }^{92}$ W. Guan, ${ }^{176}$ J. Guenther, ${ }^{65}$ F. Guescini, ${ }^{163 a}$ D. Guest, ${ }^{166}$ O. Gueta, ${ }^{155}$ B. Gui, ${ }^{113}$ E. Guido, ${ }^{53 a, 53 b}$ T. Guillemin, ${ }^{5}$ S. Guindon, ${ }^{2}$ U. Gul, ${ }^{56}$ C. Gumpert, ${ }^{32}$ J. Guo, ${ }^{36 c}$ W. Guo, ${ }^{92}$ Y. Guo, ${ }^{36 a, x}$ R. Gupta, ${ }^{43}$ S. Gupta, ${ }^{122}$ G. Gustavino, ${ }^{134 a, 134 b}$ P. Gutierrez, ${ }^{115}$ N. G. Gutierrez Ortiz ${ }^{81}$ C. Gutschow ${ }^{81}$ C. Guyot, ${ }^{138}$ M. P. Guzik, ${ }^{41 a}$ C. Gwenlan, ${ }^{122}$ C. B. Gwilliam, ${ }^{77}$ A. Haas, ${ }^{112}$ C. Haber ${ }^{16}$ H. K. Hadavand,${ }^{8}$ N. Haddad, ${ }^{137 e}$ A. Hadef, ${ }^{88}$ S. Hageböck, ${ }^{23}$ M. Hagihara, ${ }^{164}$ H. Hakobyan, ${ }^{180, a}$ M. Haleem, ${ }^{45}$ J. Haley, ${ }^{116}$ G. Halladjian, ${ }^{93}$ G. D. Hallewell, ${ }^{88}$ K. Hamacher, ${ }^{178}$ P. Hamal, ${ }^{117}$ K. Hamano, ${ }^{172}$ A. Hamilton, ${ }^{147 a}$ G. N. Hamity, ${ }^{141}$ P. G. Hamnett ${ }^{45}$ L. Han, ${ }^{36 a}$ S. Han, ${ }^{35 a, 35 d}$ K. Hanagaki, ${ }^{69, y}$ K. Hanawa, ${ }^{157}$ M. Hance, ${ }^{139}$ B. Haney, ${ }^{124}$ P. Hanke, ${ }^{60 a}$ J. B. Hansen, ${ }^{39}$ J. D. Hansen, ${ }^{39}$ M. C. Hansen, ${ }^{23}$ P. H. Hansen, ${ }^{39}$ K. Hara, ${ }^{164}$ A. S. Hard, ${ }^{176}$ T. Harenberg, ${ }^{178}$ F. Hariri, ${ }^{119}$ S. Harkusha, ${ }^{95}$ R. D. Harrington, ${ }^{49}$ P. F. Harrison, ${ }^{173}$ N. M. Hartmann, ${ }^{102}$ M. Hasegawa, ${ }^{70}$ Y. Hasegawa, ${ }^{142}$ A. Hasib, ${ }^{49}$ S. Hassani, ${ }^{138}$ S. Haug, ${ }^{18}$ R. Hauser, ${ }^{93}$ L. Hauswald ${ }^{47}$ L. B. Havener, ${ }^{38}$ M. Havranek, ${ }^{130}$ C. M. Hawkes, ${ }^{19}$ R. J. Hawkings, ${ }^{32}$ D. Hayakawa, ${ }^{159}$ D. Hayden, ${ }^{93}$ C. P. Hays, ${ }^{122}$ J. M. Hays ${ }^{79}$ H. S. Hayward, ${ }^{77}$ S. J. Haywood, ${ }^{133}$ S. J. Head, ${ }^{19}$ T. Heck, ${ }^{86}$ V. Hedberg, ${ }^{84}$ L. Heelan, ${ }^{8}$ S. Heer, ${ }^{23}$ K. K. Heidegger, ${ }^{51}$ S. Heim, ${ }^{45}$ T. Heim, ${ }^{16}$ B. Heinemann, ${ }^{45, z}$ J. J. Heinrich, ${ }^{102}$ L. Heinrich, ${ }^{112}$ C. Heinz, ${ }^{55}$ J. Hejbal, ${ }^{129}$ L. Helary, ${ }^{32}$ A. Held,${ }^{171}$ S. Hellman, ${ }^{148 a, 148 b}$ C. Helsens, ${ }^{32}$ R. C. W. Henderson, ${ }^{75}$ Y. Heng, ${ }^{176}$ S. Henkelmann, ${ }^{171}$ A. M. Henriques Correia, ${ }^{32}$ S. Henrot-Versille, ${ }^{119}$ G. H. Herbert, ${ }^{17}$ H. Herde,${ }^{25}$ V. Herget,${ }^{177}$ Y. Hernández Jiménez, ${ }^{147 c}$ H. Herr, ${ }^{86}$ G. Herten, ${ }^{51}$ R. Hertenberger, ${ }^{102}$ L. Hervas, ${ }^{32}$ T. C. Herwig, ${ }^{124}$ G. G. Hesketh, ${ }^{81}$ N. P. Hessey, ${ }^{163 a}$ J. W. Hetherly, ${ }^{43}$ S. Higashino, ${ }^{69}$ E. Higón-Rodriguez, ${ }^{170}$ K. Hildebrand, ${ }^{33}$ E. Hill, ${ }^{172}$ J. C. Hill, ${ }^{30}$ K. H. Hiller, ${ }^{45}$ S. J. Hillier, ${ }^{19}$ M. Hils, ${ }^{47}$ I. Hinchliffe, ${ }^{16}$ M. Hirose ${ }^{51}$ D. Hirschbuehl, ${ }^{178}$ B. Hiti, ${ }^{78}$ O. Hladik, ${ }^{129}$ X. Hoad,${ }^{49}$ J. Hobbs, ${ }^{150}$ N. Hod, ${ }^{163 a}$ M. C. Hodgkinson, ${ }^{141}$ P. Hodgson, ${ }^{141}$ A. Hoecker, ${ }^{32}$ M. R. Hoeferkamp, ${ }^{107}$ F. Hoenig, ${ }^{102}$ D. Hohn, ${ }^{23}$ T. R. Holmes, ${ }^{33}$ M. Homann, ${ }^{46}$ S. Honda, ${ }^{164}$ T. Honda ${ }^{69}$ T. M. Hong, ${ }^{127}$ B. H. Hooberman, ${ }^{169}$ W. H. Hopkins, ${ }^{118}$ Y. Horii, ${ }^{105}$ A. J. Horton, ${ }^{144}$ J-Y. Hostachy, ${ }^{58}$ S. Hou, ${ }^{153}$ A. Hoummada, ${ }^{137 a}$ J. Howarth, ${ }^{87}$ J. Hoya ${ }^{74}$ M. Hrabovsky, ${ }^{117}$ J. Hrdinka,${ }^{32}$ I. Hristova, ${ }^{17}$ J. Hrivnac, ${ }^{119}$ T. Hryn'ova, ${ }^{5}$ A. Hrynevich,${ }^{96}$ P. J. Hsu, ${ }^{63}$ S.-C. Hsu, ${ }^{140}$ Q. Hu,${ }^{36 a}$ S. Hu, ${ }^{36 c}$ Y. Huang, ${ }^{35 a}$ Z. Hubacek, ${ }^{130}$ F. Hubaut ${ }^{88}$ F. Huegging, ${ }^{23}$ T. B. Huffman, ${ }^{122}$ E. W. Hughes,${ }^{38}$ G. Hughes, ${ }^{75}$ M. Huhtinen,${ }^{32}$ P. Huo, ${ }^{150}$ N. Huseynov, ${ }^{68, c}$ J. Huston, ${ }^{93}$ J. Huth, ${ }^{59}$ G. Iacobucci, ${ }^{52}$ G. Iakovidis, ${ }^{27}$ I. Ibragimov, ${ }^{143}$ L. Iconomidou-Fayard, ${ }^{119}$ Z. Idrissi, ${ }^{137 e}$ P. Iengo, ${ }^{32}$ O. Igonkina, ${ }^{109, \text { aa }}$ T. Iizawa, ${ }^{174}$ Y. Ikegami, ${ }^{69}$ M. Ikeno, ${ }^{69}$ Y. Ilchenko, ${ }^{11, b b}$ D. Iliadis, ${ }^{156} \mathrm{~N}$. Ilic, ${ }^{145} \mathrm{G}$. Introzzi, ${ }^{123 a, 123 b}$ P. Ioannou, ${ }^{9, a} \mathrm{M}$. Iodice, ${ }^{136 a} \mathrm{~K}$. Iordanidou, ${ }^{38} \mathrm{~V}$. Ippolito, ${ }^{59} \mathrm{M}$. F. Isacson, ${ }^{168} \mathrm{~N}$. Ishijima, ${ }^{120} \mathrm{M}$. Ishino, ${ }^{157} \mathrm{M}$. Ishitsuka, ${ }^{159}$ C. Issever, ${ }^{122}$ S. Istin ${ }^{20 a}$ F. Ito, ${ }^{164} \mathrm{~J}$. M. Iturbe Ponce, ${ }^{62 \mathrm{a}} \mathrm{R}$. Iuppa, ${ }^{162 a, 162 b} \mathrm{H}$. Iwasaki, ${ }^{69} \mathrm{~J}$. M. Izen, ${ }^{44} \mathrm{~V}$. Izzo, ${ }^{106 a} \mathrm{~S}$. Jabbar, ${ }^{3}$ P. Jackson, ${ }^{1}$ R. M. Jacobs, ${ }^{23}$ V. Jain, ${ }^{2}$ K. B. Jakobi, ${ }^{86}$ K. Jakobs, ${ }^{51}$ S. Jakobsen, ${ }^{65}$ T. Jakoubek, ${ }^{129}$ D. O. Jamin, ${ }^{116}$

D. K. Jana, ${ }^{82}$ R. Jansky, ${ }^{52}$ J. Janssen, ${ }^{23}$ M. Janus, ${ }^{57}$ P. A. Janus, ${ }^{41 a}$ G. Jarlskog ${ }^{84}$ N. Javadov, ${ }^{68, \mathfrak{c}}$ T. Javůrek, ${ }^{51}$

M. Javurkova, ${ }^{51}$ F. Jeanneau, ${ }^{138}$ L. Jeanty, ${ }^{16}$ J. Jejelava,${ }^{54 a, c c}$ A. Jelinskas, ${ }^{173}$ P. Jenni, ${ }^{51, d d}$ C. Jeske, ${ }^{173}$ S. Jézéquel, ${ }^{5} \mathrm{H}$. Ji, ${ }^{176}$ J. Jia, ${ }^{150}$ H. Jiang, ${ }^{67}$ Y. Jiang, ${ }^{36 a}$ Z. Jiang, ${ }^{145}$ S. Jiggins, ${ }^{81}$ J. Jimenez Pena, ${ }^{170}$ S. Jin, ${ }^{35 a}$ A. Jinaru, ${ }^{28 b}$ O. Jinnouchi, ${ }^{159}$ H. Jivan, ${ }^{147 c}$ P. Johansson, ${ }^{141}$ K. A. Johns, ${ }^{7}$ C. A. Johnson, ${ }^{64}$ W. J. Johnson, ${ }^{140}$ K. Jon-And, ${ }^{148 a, 148 b}$ R. W. L. Jones, ${ }^{75}$ S. D. Jones, ${ }^{151}$ S. Jones, ${ }^{7}$ T. J. Jones,${ }^{77}$ J. Jongmanns, ${ }^{60 a}$ P. M. Jorge, ${ }^{128 a, 128 b}$ J. Jovicevic, ${ }^{163 a}$ X. Ju, ${ }^{176}$ A. Juste Rozas, ${ }^{13, w}$ M. K. Köhler, ${ }^{175}$ A. Kaczmarska, ${ }^{42}$ M. Kado ${ }^{119}$ H. Kagan, ${ }^{113}$ M. Kagan, ${ }^{145}$ S. J. Kahn, ${ }^{88}$ T. Kaji, ${ }^{174}$ E. Kajomovitz, ${ }^{48}$ C. W. Kalderon, ${ }^{84}$ A. Kaluza, ${ }^{86}$ S. Kama, ${ }^{43}$ A. Kamenshchikov, ${ }^{132}$ N. Kanaya, ${ }^{157}$ L. Kanjir, ${ }^{78}$ V. A. Kantserov, ${ }^{100}$ J. Kanzaki, ${ }^{69}$ B. Kaplan, ${ }^{112}$ L. S. Kaplan, ${ }^{176}$ D. Kar, ${ }^{147 c}$ K. Karakostas, ${ }^{10}$ N. Karastathis, ${ }^{10}$ M. J. Kareem, ${ }^{57}$ E. Karentzos,${ }^{10}$ S. N. Karpov, ${ }^{68}$ Z. M. Karpova, ${ }^{68}$ K. Karthik, ${ }^{112}$ V. Kartvelishvili, ${ }^{75}$ A. N. Karyukhin, ${ }^{132}$ K. Kasahara, ${ }^{164}$ L. Kashif, ${ }^{176}$ R. D. Kass, ${ }^{113}$ A. Kastanas, ${ }^{149}$ Y. Kataoka, ${ }^{157}$ C. Kato,${ }^{157}$ A. Katre,${ }^{52}$ J. Katzy, ${ }^{45}$ K. Kawade, ${ }^{70}$ K. Kawagoe,${ }^{73}$ T. Kawamoto, ${ }^{157}$ G. Kawamura, ${ }^{57}$ E. F. Kay, ${ }^{77}$ V. F. Kazanin, ${ }^{11, d}$ R. Keeler, ${ }^{172}$ R. Kehoe, ${ }^{43}$ J. S. Keller, ${ }^{31}$ J. J. Kempster, ${ }^{80}$ J Kendrick, ${ }^{19}$ H. Keoshkerian, ${ }^{161}$ O. Kepka, ${ }^{129}$ B. P. Kerševan, ${ }^{78}$ S. Kersten, ${ }^{178}$ R. A. Keyes, ${ }^{90}$ M. Khader, ${ }^{169}$ F. Khalil-zada, ${ }^{12}$ A. Khanov, ${ }^{116}$ A. G. Kharlamov, ${ }^{11, d}$ T. Kharlamova, ${ }^{11, d}$ A. Khodinov, ${ }^{160}$ T. J. Khoo, ${ }^{52}$ V. Khovanskiy, ${ }^{99, a}$ E. Khramov ${ }^{68}$ J. Khubua, ${ }^{54 b, e e}$ S. Kido,${ }^{70}$ C. R. Kilby, ${ }^{80}$ H. Y. Kim, ${ }^{8}$ S. H. Kim, ${ }^{164}$ Y. K. Kim, ${ }^{33}$ N. Kimura, ${ }^{156}$ O. M. Kind,${ }^{17}$ B. T. King, ${ }^{77}$ D. Kirchmeier, ${ }^{47}$ J. Kirk, ${ }^{133}$ A. E. Kiryunin, ${ }^{103}$ T. Kishimoto,${ }^{157}$ D. Kisielewska, ${ }^{41 a}$ V. Kitali, ${ }^{45}$ K. Kiuchi, ${ }^{164}$ O. Kivernyk, ${ }^{5}$ E. Kladiva, ${ }^{146 b}$ T. Klapdor-Kleingrothaus, ${ }^{51}$ M. H. Klein, ${ }^{92}$ M. Klein, ${ }^{77}$ U. Klein, ${ }^{77}$ K. Kleinknecht, ${ }^{86}$ P. Klimek, ${ }^{110}$ A. Klimentov, ${ }^{27}$ R. Klingenberg, ${ }^{46}$ T. Klingl, ${ }^{23}$ T. Klioutchnikova, ${ }^{32}$ E.-E. Kluge,${ }^{60}$ P. Kluit, ${ }^{109}$ S. Kluth, ${ }^{103}$ E. Kneringer, ${ }^{65}$ E. B. F. G. Knoops, ${ }^{88}$ A. Knue, ${ }^{103}$ A. Kobayashi, ${ }^{157}$ D. Kobayashi, ${ }^{159}$ T. Kobayashi, ${ }^{157}$ M. Kobel, ${ }^{47}$ M. Kocian, ${ }^{145}$ P. Kodys, ${ }^{131}$ T. Koffas, ${ }^{31}$ E. Koffeman, ${ }^{109}$ N. M. Köhler, ${ }^{103}$ T. Koi, ${ }^{145}$ M. Kolb, ${ }^{60 b}$ I. Koletsou, ${ }^{5}$ 
A. A. Komar, ${ }^{98, a}$ Y. Komori, ${ }^{157}$ T. Kondo, ${ }^{69}$ N. Kondrashova, ${ }^{36 c}$ K. Köneke, ${ }^{51}$ A. C. König, ${ }^{108}$ T. Kono, ${ }^{69, f f}$ R. Konoplich, ${ }^{112, \mathrm{gg}}$ N. Konstantinidis, ${ }^{81}$ R. Kopeliansky, ${ }^{64}$ S. Koperny, ${ }^{41 \mathrm{a}}$ A. K. Kopp, ${ }^{51}$ K. Korcyl, ${ }^{42}$ K. Kordas, ${ }^{156}$ A. Korn, ${ }^{81}$ A. A. Korol, ${ }^{111, d}$ I. Korolkov, ${ }^{13}$ E. V. Korolkova, ${ }^{141}$ O. Kortner, ${ }^{103}$ S. Kortner, ${ }^{103}$ T. Kosek, ${ }^{131}$ V. V. Kostyukhin, ${ }^{23}$ A. Kotwal, ${ }^{48}$ A. Koulouris, ${ }^{10}$ A. Kourkoumeli-Charalampidi, ${ }^{123 a, 123 b}$ C. Kourkoumelis, ${ }^{9}$ E. Kourlitis, ${ }^{141}$ V. Kouskoura, ${ }^{27}$ A. B. Kowalewska, ${ }^{42}$ R. Kowalewski ${ }^{172}$ T.Z. Kowalski, ${ }^{41 a}$ C. Kozakai, ${ }^{157}$ W. Kozanecki, ${ }^{138}$ A. S. Kozhin, ${ }^{132}$ V. A. Kramarenko, ${ }^{101}$ G. Kramberger, ${ }^{78}$ D. Krasnopevtsev, ${ }^{100}$ M. W. Krasny, ${ }^{83}$ A. Krasznahorkay, ${ }^{32}$ D. Krauss, ${ }^{103}$ J. A. Kremer, ${ }^{41 a}$ J. Kretzschmar, ${ }^{77}$ K. Kreutzfeldt, ${ }^{55}$ P. Krieger, ${ }^{161}$ K. Krizka, ${ }^{33}$ K. Kroeninger, ${ }^{46}$ H. Kroha, ${ }^{103}$ J. Kroll, ${ }^{129}$ J. Kroll, ${ }^{124}$ J. Kroseberg, ${ }^{23}$ J. Krstic, ${ }^{14}$ U. Kruchonak, ${ }^{68}$ H. Krüger, ${ }^{23}$ N. Krumnack, ${ }^{67}$ M. C. Kruse, ${ }^{48}$ T. Kubota, ${ }^{91}$ H. Kucuk, ${ }^{81}$ S. Kuday, ${ }^{4 b}$ J. T. Kuechler, ${ }^{178}$ S. Kuehn, ${ }^{32}$ A. Kugel, ${ }^{60 \mathrm{a}}$ F. Kuger ${ }^{177}$ T. Kuhl, ${ }^{45}$ V. Kukhtin,${ }^{68}$ R. Kukla, ${ }^{88}$ Y. Kulchitsky, ${ }^{95}$ S. Kuleshov, ${ }^{34 b}$ Y. P. Kulinich, ${ }^{169}$ M. Kuna, ${ }^{134 a, 134 b}$ T. Kunigo, ${ }^{71}$ A. Kupco, ${ }^{129}$ T. Kupfer, ${ }^{46}$ O. Kuprash, ${ }^{155}$ H. Kurashige, ${ }^{70}$ L. L. Kurchaninov, ${ }^{163 a}$ Y. A. Kurochkin, ${ }^{95}$ M. G. Kurth,${ }^{35 a, 35 d}$ V. Kus, ${ }^{129}$ E. S. Kuwertz, ${ }^{172}$ M. Kuze, ${ }^{159}$

J. Kvita, ${ }^{117}$ T. Kwan, ${ }^{172}$ D. Kyriazopoulos, ${ }^{141}$ A. La Rosa, ${ }^{103}$ J. L. La Rosa Navarro, ${ }^{26 \mathrm{~d}}$ L. La Rotonda, ${ }^{40,40 \mathrm{~b}}$ F. La Ruffa, ${ }^{40 \mathrm{a}, 40 \mathrm{~b}}$ C. Lacasta, ${ }^{170}$ F. Lacava, ${ }^{134 a, 134 \mathrm{~b}}$ J. Lacey ${ }^{45}$ H. Lacker, ${ }^{17}$ D. Lacour, ${ }^{83}$ E. Ladygin, ${ }^{68}$ R. Lafaye,${ }^{5}$ B. Laforge, ${ }^{83}$ T. Lagouri, ${ }^{179}$ S. Lai, ${ }^{57}$ S. Lammers, ${ }^{64}$ W. Lampl, ${ }^{7}$ E. Lançon, ${ }^{27}$ U. Landgraf, ${ }^{51}$ M. P. J. Landon, ${ }^{79}$ M. C. Lanfermann, ${ }^{52}$ V. S. Lang, ${ }^{60 a}$ J. C. Lange, ${ }^{13}$ R. J. Langenberg, ${ }^{32}$ A. J. Lankford, ${ }^{166}$ F. Lanni, ${ }^{27}$ K. Lantzsch, ${ }^{23}$ A. Lanza, ${ }^{123 a}$ A. Lapertosa, ${ }^{53,53 b}$ S. Laplace, ${ }^{83}$ J. F. Laporte, ${ }^{138}$ T. Lari, ${ }^{94 a}$ F. Lasagni Manghi, ${ }^{22 a, 22 b}$ M. Lassnig, ${ }^{32}$ P. Laurelli, ${ }^{50}$ W. Lavrijsen, ${ }^{16}$ A. T. Law,${ }^{139}$ P. Laycock, ${ }^{77}$ T. Lazovich, ${ }^{59}$ M. Lazzaroni, ${ }^{94 a, 94 b}$ B. Le, ${ }^{91}$ O. Le Dortz, ${ }^{83}$ E. Le Guirriec ${ }^{88}$ E. P. Le Quilleuc, ${ }^{138}$ M. LeBlanc, ${ }^{172}$ T. LeCompte,${ }^{6}$ F. Ledroit-Guillon, ${ }^{58}$ C. A. Lee, ${ }^{27}$ G. R. Lee, ${ }^{133, \text { hh }}$ S. C. Lee, ${ }^{153}$ L. Lee, ${ }^{59}$ B. Lefebvre, ${ }^{90}$ G. Lefebvre, ${ }^{83}$ M. Lefebvre, ${ }^{172}$ F. Legger, ${ }^{102}$ C. Leggett, ${ }^{16}$ G. Lehmann Miotto, ${ }^{32}$ X. Lei, ${ }^{7}$ W. A. Leight, ${ }^{45}$ M. A. L. Leite, ${ }^{26 d}$ R. Leitner, ${ }^{131}$ D. Lellouch, ${ }^{175}$ B. Lemmer, ${ }^{57}$ K. J. C. Leney, ${ }^{81}$ T. Lenz, ${ }^{23}$ B. Lenzi, ${ }^{32}$ R. Leone, ${ }^{7}$ S. Leone, ${ }^{126 a, 126 b}$ C. Leonidopoulos, ${ }^{49}$ G. Lerner, ${ }^{151}$ C. Leroy,${ }^{97}$ A. A. J. Lesage, ${ }^{138}$ C. G. Lester,${ }^{30}$ M. Levchenko, ${ }^{125}$ J. Levêque, ${ }^{5}$ D. Levin, ${ }^{92}$ L. J. Levinson, ${ }^{175}$ M. Levy, ${ }^{19}$ D. Lewis, ${ }^{79}$ B. Li, ${ }^{36 a, x}$ Changqiao Li, ${ }^{36 a} \mathrm{H}^{2} \mathrm{Li},{ }^{150}$ L. Li ${ }^{36 \mathrm{c}}$ Q. Li ${ }^{35 a, 35 \mathrm{~d}} \mathrm{~S} . \mathrm{Li},{ }^{48}$ X. Li, ${ }^{36 \mathrm{c}}$ Y. Li ${ }^{143}$ Z. Liang, ${ }^{35 \mathrm{a}}$ B. Liberti, ${ }^{135 \mathrm{a}}$ A. Liblong, ${ }^{161} \mathrm{~K}$. Lie, ${ }^{6 \mathrm{c}} \mathrm{J}$. Liebal, ${ }^{23} \mathrm{~W}$. Liebig, ${ }^{15}$ A. Limosani, ${ }^{152}$ S. C. Lin, ${ }^{182}$ T. H. Lin, ${ }^{86}$ R. A. Linck, ${ }^{64}$ B. E. Lindquist, ${ }^{150}$ A. E. Lionti, ${ }^{52}$ E. Lipeles, ${ }^{124}$ A. Lipniacka, ${ }^{15}$ M. Lisovyi, ${ }^{60 b}$ T. M. Liss, ${ }^{169, \text { ii }}$ A. Lister, ${ }^{171}$ A. M. Litke, ${ }^{139}$ B. Liu, ${ }^{153, j j}$ H. Liu, ${ }^{92}$ H. Liu, ${ }^{27}$ J. K. K. Liu, ${ }^{122}$ J. Liu, ${ }^{36 b}$ J. B. Liu, ${ }^{36 a}$ K. Liu, ${ }^{88}$ L. Liu, ${ }^{169}$ M. Liu, ${ }^{36 a}$ Y. L. Liu, ${ }^{36 a}$ Y. Liu, ${ }^{36 a}$ M. Livan, ${ }^{123 a, 123 b}$ A. Lleres, ${ }^{58}$ J. Llorente Merino, ${ }^{35 a}$ S. L. Lloyd,${ }^{79}$ C. Y. Lo, ${ }^{62 b}$ F. Lo Sterzo, ${ }^{153}$ E. M. Lobodzinska,${ }^{45}$ P. Loch, ${ }^{7}$ F. K. Loebinger, ${ }^{87}$ A. Loesle ${ }^{51}$ K. M. Loew, ${ }^{25}$ A. Loginov, ${ }^{179, a}$ T. Lohse, ${ }^{17}$ K. Lohwasser, ${ }^{141}$ M. Lokajicek, ${ }^{129}$ B. A. Long, ${ }^{24}$ J. D. Long, ${ }^{169}$ R. E. Long, ${ }^{75}$ L. Longo ${ }^{76 a, 76 b}$ K. A. Looper, ${ }^{113}$ J. A. Lopez, ${ }^{34 b}$ D. Lopez Mateos, ${ }^{59}$ I. Lopez Paz, ${ }^{13}$ A. Lopez Solis,${ }^{83}$ J. Lorenz, ${ }^{102}$ N. Lorenzo Martinez, ${ }^{5}$ M. Losada, ${ }^{21}$ P. J. Lösel, ${ }^{102}$ X. Lou, ${ }^{35 a}$ A. Lounis, ${ }^{119}$ J. Love, ${ }^{6}$ P. A. Love,${ }^{75}$ H. Lu, ${ }^{62 a}$ N. Lu, ${ }^{92}$ Y. J. Lu ${ }^{63}$ H. J. Lubatti, ${ }^{140}$ C. Luci, ${ }^{134 a, 134 b}$ A. Lucotte ${ }^{58}$ C. Luedtke, ${ }^{51}$ F. Luehring, ${ }^{64}$ W. Lukas, ${ }^{65}$ L. Luminari, ${ }^{134 a}$ O. Lundberg, ${ }^{148 a, 148 b}$

B. Lund-Jensen, ${ }^{149}$ M. S. Lutz, ${ }^{89}$ P. M. Luzi, ${ }^{83}$ D. Lynn, ${ }^{27}$ R. Lysak, ${ }^{129}$ E. Lytken, ${ }^{84}$ F. Lyu, ${ }^{35 a}$ V. Lyubushkin,${ }^{68}$ H. Ma ${ }^{27}$ L. L. Ma ${ }^{36 \mathrm{~b}}$ Y. Ma, ${ }^{36 \mathrm{~b}}$ G. Maccarrone, ${ }^{50}$ A. Macchiolo, ${ }^{103}$ C. M. Macdonald, ${ }^{141}$ B. Maček, ${ }^{78}$ J. Machado Miguens, ${ }^{124,128 \mathrm{~b}}$ D. Madaffari, ${ }^{170}$ R. Madar, ${ }^{37}$ W. F. Mader, ${ }^{47}$ A. Madsen, ${ }^{45}$ J. Maeda ${ }^{70}$ S. Maeland,${ }^{15}$ T. Maeno, ${ }^{27}$ A. S. Maevskiy, ${ }^{101}$

V. Magerl,${ }^{51}$ J. Mahlstedt, ${ }^{109}$ C. Maiani, ${ }^{119}$ C. Maidantchik, ${ }^{26 a}$ A. A. Maier, ${ }^{103}$ T. Maier, ${ }^{102}$ A. Maio, ${ }^{128 a, 128 b, 128 d}$

O. Majersky, ${ }^{146 a}$ S. Majewski, ${ }^{118}$ Y. Makida, ${ }^{69}$ N. Makovec, ${ }^{119}$ B. Malaescu, ${ }^{83}$ Pa. Malecki, ${ }^{42}$ V. P. Maleev, ${ }^{125}$ F. Malek, ${ }^{58}$ U. Mallik, ${ }^{66}$ D. Malon, ${ }^{6}$ C. Malone, ${ }^{30}$ S. Maltezos, ${ }^{10}$ S. Malyukov, ${ }^{32}$ J. Mamuzic, ${ }^{170}$ G. Mancini,${ }^{50}$ I. Mandić ${ }^{78}$ J. Maneira, ${ }^{128 a, 128 b}$ L. Manhaes de Andrade Filho, ${ }^{26 b}$ J. Manjarres Ramos,${ }^{47}$ K. H. Mankinen, ${ }^{84}$ A. Mann, ${ }^{102}$ A. Manousos ${ }^{32}$ B. Mansoulie, ${ }^{138}$ J. D. Mansour, ${ }^{35 a}$ R. Mantifel, ${ }^{90}$ M. Mantoani, ${ }^{57}$ S. Manzoni, ${ }^{94 a, 94 b}$ L. Mapelli, ${ }^{32}$ G. Marceca, ${ }^{29}$ L. March,${ }^{52}$ L. Marchese, ${ }^{122}$ G. Marchiori, ${ }^{83}$ M. Marcisovsky, ${ }^{129}$ M. Marjanovic, ${ }^{37}$ D. E. Marley, ${ }^{92}$ F. Marroquim, ${ }^{26 a}$ S. P. Marsden, ${ }^{87}$ Z. Marshall, ${ }^{16}$ M. U. F Martensson, ${ }^{168}$ S. Marti-Garcia, ${ }^{170}$ C. B. Martin, ${ }^{113}$ T. A. Martin, ${ }^{173}$ V. J. Martin, ${ }^{49}$

B. Martin dit Latour, ${ }^{15}$ M. Martinez, ${ }^{13, w}$ V. I. Martinez Outschoorn, ${ }^{169}$ S. Martin-Haugh, ${ }^{133}$ V. S. Martoiu, ${ }^{28 b}$ A. C. Martyniuk, ${ }^{81}$ A. Marzin,${ }^{32}$ L. Masetti, ${ }^{86}$ T. Mashimo, ${ }^{157}$ R. Mashinistov, ${ }^{98}$ J. Masik, ${ }^{87}$ A. L. Maslennikov, ${ }^{11, d}$ L. Massa, ${ }^{135 a, 135 b}$ P. Mastrandrea ${ }^{5}$ A. Mastroberardino, ${ }^{40 a, 40 b}$ T. Masubuchi, ${ }^{157}$ P. Mättig, ${ }^{178}$ J. Maurer ${ }^{28 b}$ S. J. Maxfield, ${ }^{77}$

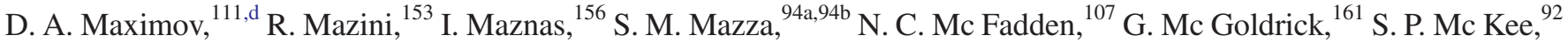

A. McCarn, ${ }^{92}$ R. L. McCarthy, ${ }^{150}$ T. G. McCarthy, ${ }^{103}$ L. I. McClymont, ${ }^{81}$ E. F. McDonald, ${ }^{91}$ J. A. Mcfayden,${ }^{81}$

G. Mchedlidze, ${ }^{57}$ S. J. McMahon, ${ }^{133}$ P. C. McNamara, ${ }^{91}$ R. A. McPherson, ${ }^{172, p}$ S. Meehan, ${ }^{140}$ T. J. Megy, ${ }^{51}$ S. Mehlhase, ${ }^{102}$ A. Mehta ${ }^{77}$ T. Meideck,${ }^{58}$ K. Meier ${ }^{60 \mathrm{a}}$ B. Meirose, ${ }^{44}$ D. Melini, ${ }^{170, k \mathrm{kk}}$ B. R. Mellado Garcia, ${ }^{147 \mathrm{c}}$ J. D. Mellenthin, ${ }^{57}$ M. Melo, ${ }^{146 a}$ F. Meloni, ${ }^{18}$ A. Melzer, ${ }^{23}$ S. B. Menary, ${ }^{87}$ L. Meng, ${ }^{77}$ X. T. Meng, ${ }^{92}$ A. Mengarelli, ${ }^{22 a, 22 b}$ S. Menke, ${ }^{103}$ 
E. Meoni, ${ }^{40 a, 40 b}$ S. Mergelmeyer, ${ }^{17}$ P. Mermod, ${ }^{52}$ L. Merola, ${ }^{106 a, 106 b}$ C. Meroni,${ }^{94 a}$ F. S. Merritt, ${ }^{33}$ A. Messina, ${ }^{134 a, 134 b}$ J. Metcalfe, ${ }^{6}$ A. S. Mete, ${ }^{166}$ C. Meyer, ${ }^{124}$ J-P. Meyer, ${ }^{138}$ J. Meyer, ${ }^{109}$ H. Meyer Zu Theenhausen, ${ }^{60 a}$ F. Miano, ${ }^{151}$ R. P. Middleton, ${ }^{133}$ S. Miglioranzi, ${ }^{53 a, 53 b}$ L. Mijović, ${ }^{49}$ G. Mikenberg, ${ }^{175}$ M. Mikestikova, ${ }^{129}$ M. Mikuž, ${ }^{78}$ M. Milesi, ${ }^{91}$

A. Milic, ${ }^{161}$ D. W. Miller, ${ }^{33}$ C. Mills, ${ }^{49}$ A. Milov, ${ }^{175}$ D. A. Milstead, ${ }^{148 a, 148 b}$ A. A. Minaenko, ${ }^{132}$ Y. Minami, ${ }^{157}$

I. A. Minashvili, ${ }^{54 b}$ A. I. Mincer, ${ }^{112}$ B. Mindur, ${ }^{41 a}$ M. Mineev ${ }^{68}$ Y. Minegishi, ${ }^{157}$ Y. Ming,${ }^{176}$ L. M. Mir, ${ }^{13}$ K. P. Mistry, ${ }^{124}$

T. Mitani, ${ }^{174}$ J. Mitrevski, ${ }^{102}$ V. A. Mitsou, ${ }^{170}$ A. Miucci, ${ }^{18}$ P. S. Miyagawa, ${ }^{141}$ A. Mizukami, ${ }^{69}$ J. U. Mjörnmark ${ }^{84}$

T. Mkrtchyan, ${ }^{180}$ M. Mlynarikova, ${ }^{131}$ T. Moa, ${ }^{148 a, 148 b}$ K. Mochizuki, ${ }^{97}$ P. Mogg,${ }^{51}$ S. Mohapatra ${ }^{38}$ S. Molander, ${ }^{148 a, 148 b}$

R. Moles-Valls, ${ }^{23}$ R. Monden, ${ }^{71}$ M. C. Mondragon, ${ }^{93}$ K. Mönig, ${ }^{45}$ J. Monk, ${ }^{39}$ E. Monnier, ${ }^{88}$ A. Montalbano, ${ }^{150}$

J. Montejo Berlingen, ${ }^{32}$ F. Monticelli, ${ }^{74}$ S. Monzani, ${ }^{94 a},{ }^{44 b}$ R. W. Moore, ${ }^{3}$ N. Morange,${ }^{119}$ D. Moreno, ${ }^{21}$ M. Moreno Llácer, ${ }^{32}$ P. Morettini, ${ }^{53 a}$ S. Morgenstern, ${ }^{32}$ D. Mori, ${ }^{144}$ T. Mori ${ }^{157}$ M. Morii, ${ }^{59}$ M. Morinaga,${ }^{157}$ V. Morisbak,${ }^{121}$ A. K. Morley, ${ }^{32}$

G. Mornacchi, ${ }^{32}$ J. D. Morris, ${ }^{79}$ L. Morvaj, ${ }^{150}$ P. Moschovakos, ${ }^{10}$ M. Mosidze ${ }^{54 b}$ H. J. Moss, ${ }^{141}$ J. Moss, ${ }^{145,11}$

K. Motohashi, ${ }^{159}$ R. Mount, ${ }^{145}$ E. Mountricha, ${ }^{27}$ E. J. W. Moyse, ${ }^{89}$ S. Muanza,${ }^{88}$ F. Mueller, ${ }^{103}$ J. Mueller, ${ }^{127}$

R. S. P. Mueller, ${ }^{102}$ D. Muenstermann, ${ }^{75}$ P. Mullen, ${ }^{56}$ G. A. Mullier, ${ }^{18}$ F. J. Munoz Sanchez, ${ }^{87}$ W. J. Murray,${ }^{173,133}$

H. Musheghyan, ${ }^{32}$ M. Muškinja, ${ }^{78}$ A. G. Myagkov, ${ }^{132, m m}$ M. Myska, ${ }^{130}$ B. P. Nachman, ${ }^{16}$ O. Nackenhorst,${ }^{52}$ K. Nagai, ${ }^{122}$

R. Nagai, ${ }^{69, f f}$ K. Nagano, ${ }^{69}$ Y. Nagasaka, ${ }^{61}$ K. Nagata, ${ }^{164}$ M. Nagel,${ }^{51}$ E. Nagy, ${ }^{88}$ A. M. Nairz, ${ }^{32}$ Y. Nakahama, ${ }^{105}$

K. Nakamura, ${ }^{69}$ T. Nakamura, ${ }^{157}$ I. Nakano, ${ }^{114}$ R. F. Naranjo Garcia, ${ }^{45}$ R. Narayan, ${ }^{11}$ D. I. Narrias Villar, ${ }^{60 a}$ I. Naryshkin, ${ }^{125}$

T. Naumann, ${ }^{45}$ G. Navarro, ${ }^{21}$ R. Nayyar, ${ }^{7}$ H. A. Neal, ${ }^{92}$ P. Yu. Nechaeva, ${ }^{98}$ T. J. Neep ${ }^{138}$ A. Negri, ${ }^{123 a, 123 b}$ M. Negrini, ${ }^{22 a}$

S. Nektarijevic, ${ }^{108}$ C. Nellist, ${ }^{119}$ A. Nelson, ${ }^{166}$ M. E. Nelson, ${ }^{122}$ S. Nemecek, ${ }^{129}$ P. Nemethy, ${ }^{112}$ M. Nessi, ${ }^{32, n n}$

M. S. Neubauer, ${ }^{169}$ M. Neumann, ${ }^{178}$ P. R. Newman, ${ }^{19}$ T. Y. Ng, ${ }^{62 \mathrm{c}}$ T. Nguyen Manh, ${ }^{97}$ R. B. Nickerson, ${ }^{122}$ R. Nicolaidou, ${ }^{138}$ J. Nielsen, ${ }^{139}$ V. Nikolaenko, ${ }^{132, \mathrm{~mm}}$ I. Nikolic-Audit ${ }^{83}$ K. Nikolopoulos, ${ }^{19}$ J. K. Nilsen, ${ }^{121}$ P. Nilsson, ${ }^{27}$ Y. Ninomiya, ${ }^{157}$ A. Nisati, ${ }^{134 a}$ N. Nishu, ${ }^{35 c}$ R. Nisius, ${ }^{103}$ I. Nitsche, ${ }^{46}$ T. Nitta,${ }^{174}$ T. Nobe, ${ }^{157}$ Y. Noguchi, ${ }^{71}$ M. Nomachi, ${ }^{120}$ I. Nomidis, ${ }^{31}$ M. A. Nomura, ${ }^{27}$ T. Nooney, ${ }^{79}$ M. Nordberg, ${ }^{32}$ N. Norjoharuddeen, ${ }^{122}$ O. Novgorodova, ${ }^{47}$ M. Nozaki, ${ }^{69}$ L. Nozka, ${ }^{117}$ K. Ntekas, ${ }^{166}$ E. Nurse, ${ }^{81}$ F. Nuti, ${ }^{91}$ K. O' connor, ${ }^{25}$ D. C. O’Neil,${ }^{144}$ A. A. O'Rourke, ${ }^{45}$ V. O'Shea ${ }^{56}$ F. G. Oakham, ${ }^{31, e}$ H. Oberlack, ${ }^{103}$ T. Obermann, ${ }^{23}$ J. Ocariz, ${ }^{83}$ A. Ochi, ${ }^{70}$ I. Ochoa, ${ }^{38}$ J. P. Ochoa-Ricoux, ${ }^{34 a}$ S. Oda, ${ }^{73}$ S. Odaka, ${ }^{69}$ A. Oh,${ }^{87}$

S. H. Oh, ${ }^{48}$ C. C. Ohm, ${ }^{16}$ H. Ohman, ${ }^{168}$ H. Oide, ${ }^{53 a, 53 b}$ H. Okawa, ${ }^{164}$ Y. Okumura, ${ }^{157}$ T. Okuyama, ${ }^{69}$ A. Olariu, ${ }^{28 b}$ L. F. Oleiro Seabra, ${ }^{128 a}$ S. A. Olivares Pino, ${ }^{49}$ D. Oliveira Damazio, ${ }^{27}$ A. Olszewski, ${ }^{42}$ J. Olszowska, ${ }^{42}$ A. Onofre, ${ }^{128 a}, 128 \mathrm{e}$ K. Onogi, ${ }^{105}$ P. U. E. Onyisi, ${ }^{11, b b}$ H. Oppen, ${ }^{121}$ M. J. Oreglia, ${ }^{33}$ Y. Oren, ${ }^{155}$ D. Orestano, ${ }^{136 a, 136 b}$ N. Orlando, ${ }^{62 b}$ R. S. Orr, ${ }^{161}$ B. Osculati, ${ }^{53 a, 53 b, a}$ R. Ospanov, ${ }^{36 a}$ G. Otero y Garzon, ${ }^{29}$ H. Otono,${ }^{73}$ M. Ouchrif, ${ }^{137 d}$ F. Ould-Saada, ${ }^{121}$ A. Ouraou, ${ }^{138}$ K. P. Oussoren, ${ }^{109}$ Q. Ouyang, ${ }^{35 a}$ M. Owen, ${ }^{56}$ R. E. Owen, ${ }^{19}$ V. E. Ozcan ${ }^{20 a}$ N. Ozturk, ${ }^{8}$ K. Pachal, ${ }^{144}$ A. Pacheco Pages,${ }^{13}$ L. Pacheco Rodriguez, ${ }^{138}$ C. Padilla Aranda,${ }^{13}$ S. Pagan Griso,${ }^{16}$ M. Paganini, ${ }^{179}$ F. Paige, ${ }^{27}$ G. Palacino, ${ }^{64}$ S. Palazzo, ${ }^{40 a, 40 b}$ S. Palestini, ${ }^{32}$ M. Palka, ${ }^{4 b}$ D. Pallin, ${ }^{37}$ E. St. Panagiotopoulou, ${ }^{10}$ I. Panagoulias, ${ }^{10}$ C. E. Pandini, ${ }^{126 a, 126 b}$

J. G. Panduro Vazquez, ${ }^{80}$ P. Pani, ${ }^{32}$ S. Panitkin, ${ }^{27}$ D. Pantea ${ }^{28 b}$ L. Paolozzi, ${ }^{52}$ Th. D. Papadopoulou, ${ }^{10}$ K. Papageorgiou, ${ }^{9, t}$ A. Paramonov, ${ }^{6}$ D. Paredes Hernandez, ${ }^{179}$ A. J. Parker, ${ }^{75}$ M. A. Parker, ${ }^{30}$ K. A. Parker ${ }^{45}$ F. Parodi, ${ }^{53 a, 53 b}$ J. A. Parsons,${ }^{38}$ U. Parzefall, ${ }^{51}$ V. R. Pascuzzi, ${ }^{161}$ J. M. Pasner, ${ }^{139}$ E. Pasqualucci,${ }^{134 a}$ S. Passaggio ${ }^{53 a}$ Fr. Pastore, ${ }^{80}$ S. Pataraia,${ }^{86}$ J. R. Pater ${ }^{87}$

T. Pauly, ${ }^{32}$ B. Pearson, ${ }^{103}$ S. Pedraza Lopez, ${ }^{170}$ R. Pedro, ${ }^{128 a, 128 b}$ S. V. Peleganchuk,${ }^{111, d}$ O. Penc, ${ }^{129}$ C. Peng, ${ }^{35 a, 35 d}$ H. Peng, ${ }^{36 a}$ J. Penwell, ${ }^{64}$ B. S. Peralva, ${ }^{26 b}$ M. M. Perego, ${ }^{138}$ D. V. Perepelitsa, ${ }^{27}$ F. Peri, ${ }^{17}$ L. Perini,${ }^{94 a, 94 b}$ H. Pernegger, ${ }^{32}$ S. Perrella, ${ }^{106 a, 106 b}$ R. Peschke, ${ }^{45}$ V. D. Peshekhonov, ${ }^{68, a}$ K. Peters,${ }^{45}$ R. F. Y. Peters, ${ }^{87}$ B. A. Petersen, ${ }^{32}$ T. C. Petersen, ${ }^{39}$ E. Petit, ${ }^{58}$ A. Petridis, ${ }^{1}$ C. Petridou, ${ }^{156}$ P. Petroff, ${ }^{119}$ E. Petrolo, ${ }^{134 a}$ M. Petrov, ${ }^{122}$ F. Petrucci,${ }^{136 a, 136 b}$ N. E. Pettersson, ${ }^{89}$ A. Peyaud ${ }^{138}$ R. Pezoa, ${ }^{34 b}$ F. H. Phillips,${ }^{93}$ P. W. Phillips, ${ }^{133}$ G. Piacquadio,${ }^{150}$ E. Pianori, ${ }^{173}$ A. Picazio, ${ }^{89}$ E. Piccaro, ${ }^{79}$ M. A. Pickering, ${ }^{122}$ R. Piegaia, ${ }^{29}$ J. E. Pilcher, ${ }^{33}$ A. D. Pilkington, ${ }^{87}$ A. W. J. Pin, ${ }^{87}$ M. Pinamonti, ${ }^{135 a, 135 b}$ J. L. Pinfold, ${ }^{3}$ H. Pirumov, ${ }^{45}$ M. Pitt, ${ }^{175}$ L. Plazak, ${ }^{146 a}$ M.-A. Pleier,${ }^{27}$ V. Pleskot, ${ }^{86}$ E. Plotnikova, ${ }^{68}$ D. Pluth, ${ }^{67}$ P. Podberezko, ${ }^{111}$ R. Poettgen, ${ }^{148 a, 148 b}$ R. Poggi, ${ }^{123 a, 123 b}$ L. Poggioli, ${ }^{119}$ D. Pohl,${ }^{23}$ G. Polesello, ${ }^{123 a}$ A. Poley, ${ }^{45}$ A. Policicchio, ${ }^{40 a, 40 b}$ R. Polifka, ${ }^{32}$ A. Polini, ${ }^{22 a}$ C. S. Pollard, ${ }^{56}$ V. Polychronakos,${ }^{27}$ K. Pommès, ${ }^{32}$ D. Ponomarenko, ${ }^{100}$ L. Pontecorvo, ${ }^{134 a}$ G. A. Popeneciu, ${ }^{28 d}$ A. Poppleton, ${ }^{32}$ S. Pospisil, ${ }^{130}$ K. Potamianos, ${ }^{16}$ I. N. Potrap,${ }^{68}$ C. J. Potter,${ }^{30}$ G. Poulard,${ }^{32}$ T. Poulsen,${ }^{84}$ J. Poveda, ${ }^{32}$ M. E. Pozo Astigarraga, ${ }^{32}$ P. Pralavorio, ${ }^{88}$ A. Pranko, ${ }^{16}$ S. Prell,${ }^{67}$ D. Price, ${ }^{87}$ M. Primavera, ${ }^{76 a}$ S. Prince,${ }^{90}$ N. Proklova, ${ }^{100}$ K. Prokofiev, ${ }^{62 c}$ F. Prokoshin, ${ }^{34 b}$ S. Protopopescu, ${ }^{27}$ J. Proudfoot, ${ }^{6}$ M. Przybycien, ${ }^{41 a}$ A. Puri, ${ }^{169}$ P. Puzo, ${ }^{119}$ J. Qian, ${ }^{92}$ G. Qin, ${ }^{56}$ Y. Qin ${ }^{87}$ A. Quadt,${ }^{57}$ M. Queitsch-Maitland, ${ }^{45}$ D. Quilty, ${ }^{56}$ S. Raddum, ${ }^{121}$ V. Radeka, ${ }^{27}$ V. Radescu, ${ }^{122}$ S. K. Radhakrishnan, ${ }^{150}$ P. Radloff, ${ }^{118}$ P. Rados, ${ }^{91}$ F. Ragusa, ${ }^{94 a, 94 b}$ G. Rahal, ${ }^{181}$ J. A. Raine, ${ }^{87}$ S. Rajagopalan ${ }^{27}$ C. Rangel-Smith, ${ }^{168}$ T. Rashid, ${ }^{119}$ S. Raspopov, ${ }^{5}$ M. G. Ratti, ${ }^{44 a}, 94 b$ D. M. Rauch, ${ }^{45}$ F. Rauscher, ${ }^{102}$ S. Rave, ${ }^{86}$ 
I. Ravinovich, ${ }^{175}$ J. H. Rawling, ${ }^{87}$ M. Raymond ${ }^{32}$ A. L. Read ${ }^{121}$ N. P. Readioff,${ }^{58}$ M. Reale, ${ }^{76 a, 76 b}$ D. M. Rebuzzi, ${ }^{123 a, 123 b}$ A. Redelbach, ${ }^{177}$ G. Redlinger, ${ }^{27}$ R. Reece, ${ }^{139}$ R. G. Reed,${ }^{147 \mathrm{c}}$ K. Reeves, ${ }^{44}$ L. Rehnisch, ${ }^{17}$ J. Reichert, ${ }^{124}$ A. Reiss ${ }^{86}$ C. Rembser ${ }^{32}$ H. Ren, ${ }^{35 a, 35 d}$ M. Rescigno, ${ }^{134 a}$ S. Resconi, ${ }^{94 a}$ E. D. Resseguie, ${ }^{124}$ S. Rettie, ${ }^{171}$ E. Reynolds, ${ }^{19}$ O. L. Rezanova, ${ }^{111, \mathrm{~d}}$ P. Reznicek, ${ }^{131}$ R. Rezvani, ${ }^{97}$ R. Richter, ${ }^{103}$ S. Richter, ${ }^{81}$ E. Richter-Was, ${ }^{41 \mathrm{~b}}$ O. Ricken, ${ }^{23}$ M. Ridel, ${ }^{83}$ P. Rieck, ${ }^{103}$ C. J. Riegel, ${ }^{178}$ J. Rieger,${ }^{57}$ O. Rifki, ${ }^{115}$ M. Rijssenbeek, ${ }^{150}$ A. Rimoldi, ${ }^{123 a, 123 b}$ M. Rimoldi, ${ }^{18}$ L. Rinaldi, ${ }^{22 a}$ G. Ripellino, ${ }^{149}$ B. Ristić, ${ }^{32}$ E. Ritsch, ${ }^{32}$ I. Riu, ${ }^{13}$ F. Rizatdinova, ${ }^{116}$ E. Rizvi, ${ }^{79}$ C. Rizzi, ${ }^{13}$ R. T. Roberts, ${ }^{87}$

S. H. Robertson, ${ }^{90, p}$ A. Robichaud-Veronneau, ${ }^{90}$ D. Robinson, ${ }^{30}$ J. E. M. Robinson, ${ }^{45}$ A. Robson,${ }^{56}$ E. Rocco, ${ }^{86}$ C. Roda, ${ }^{126 a, 126 b}$ Y. Rodina, ${ }^{88,00}$ S. Rodriguez Bosca, ${ }^{170}$ A. Rodriguez Perez, ${ }^{13}$ D. Rodriguez Rodriguez, ${ }^{170}$ S. Roe, ${ }^{32}$

C. S. Rogan, ${ }^{59}$ O. Røhne, ${ }^{121}$ J. Roloff, ${ }^{59}$ A. Romaniouk, ${ }^{100}$ M. Romano, ${ }^{22 a, 22 b}$ S. M. Romano Saez, ${ }^{37}$ E. Romero Adam, ${ }^{170}$ N. Rompotis, ${ }^{77}$ M. Ronzani, ${ }^{51}$ L. Roos, ${ }^{83}$ S. Rosati, ${ }^{134 a}$ K. Rosbach, ${ }^{51}$ P. Rose, ${ }^{139}$ N.-A. Rosien, ${ }^{57}$ E. Rossi, ${ }^{106 a, 106 b}$

L. P. Rossi, ${ }^{53 \mathrm{a}}$ J. H. N. Rosten, ${ }^{30}$ R. Rosten, ${ }^{140}$ M. Rotaru, ${ }^{28 \mathrm{~b}}$ J. Rothberg, ${ }^{140}$ D. Rousseau, ${ }^{119}$ A. Rozanov, ${ }^{88}$ Y. Rozen, ${ }^{154}$ X. Ruan, ${ }^{147 c}$ F. Rubbo, ${ }^{145}$ F. Rühr, ${ }^{51}$ A. Ruiz-Martinez, ${ }^{31}$ Z. Rurikova, ${ }^{51}$ N. A. Rusakovich, ${ }^{68}$ H. L. Russell, ${ }^{90}$

J. P. Rutherfoord, ${ }^{7}$ N. Ruthmann, ${ }^{32}$ Y. F. Ryabov, ${ }^{125}$ M. Rybar, ${ }^{169}$ G. Rybkin, ${ }^{119}$ S. Ryu, ${ }^{6}$ A. Ryzhov, ${ }^{132}$ G. F. Rzehorz, ${ }^{57}$ A. F. Saavedra, ${ }^{152}$ G. Sabato, ${ }^{109}$ S. Sacerdoti, ${ }^{29}$ H. F-W. Sadrozinski, ${ }^{139}$ R. Sadykov, ${ }^{68}$ F. Safai Tehrani, ${ }^{134 a}$ P. Saha, ${ }^{110}$ M. Sahinsoy, ${ }^{60 \mathrm{a}}$ M. Saimpert, ${ }^{45}$ M. Saito, ${ }^{157}$ T. Saito, ${ }^{157}$ H. Sakamoto,${ }^{157}$ Y. Sakurai,${ }^{174}$ G. Salamanna, ${ }^{136 a, 136 \mathrm{~b}}$

J. E. Salazar Loyola, ${ }^{34 \mathrm{~b}}$ D. Salek, ${ }^{109}$ P. H. Sales De Bruin, ${ }^{168}$ D. Salihagic, ${ }^{103}$ A. Salnikov, ${ }^{145}$ J. Salt, ${ }^{170}$ D. Salvatore, ${ }^{40 \mathrm{a}, 40 \mathrm{~b}}$ F. Salvatore, ${ }^{151}$ A. Salvucci, ${ }^{62 a, 62 b, 62 c}$ A. Salzburger,${ }^{32}$ D. Sammel,${ }^{51}$ D. Sampsonidis, ${ }^{156}$ D. Sampsonidou, ${ }^{156}$ J. Sánchez, ${ }^{170}$ V. Sanchez Martinez, ${ }^{170}$ A. Sanchez Pineda, ${ }^{167 a, 167 c}$ H. Sandaker, ${ }^{121}$ R. L. Sandbach, ${ }^{79}$ C. O. Sander, ${ }^{45}$ M. Sandhoff, ${ }^{178}$ C. Sandoval, ${ }^{21}$ D. P. C. Sankey, ${ }^{133}$ M. Sannino,${ }^{53 a, 53 b}$ Y. Sano, ${ }^{105}$ A. Sansoni,${ }^{50}$ C. Santoni, ${ }^{37}$ H. Santos, ${ }^{128 a}$ I. Santoyo Castillo, ${ }^{151}$ A. Sapronov, ${ }^{68}$ J. G. Saraiva, ${ }^{128 a, 128 d}$ B. Sarrazin, ${ }^{23}$ O. Sasaki, ${ }^{69}$ K. Sato, ${ }^{164}$ E. Sauvan, ${ }^{5}$ G. Savage, ${ }^{80}$ P. Savard, ${ }^{161, \mathrm{e}}$ N. Savic, ${ }^{103}$ C. Sawyer, ${ }^{133}$ L. Sawyer, ${ }^{82, \mathrm{v}}$ J. Saxon, ${ }^{33}$ C. Sbarra, ${ }^{22 \mathrm{a}}$ A. Sbrizzi, ${ }^{22 a, 22 b}$ T. Scanlon, ${ }^{81}$

D. A. Scannicchio, ${ }^{166}$ M. Scarcella, ${ }^{152}$ J. Schaarschmidt, ${ }^{140}$ P. Schacht, ${ }^{103}$ B. M. Schachtner, ${ }^{102}$ D. Schaefer, ${ }^{32}$ L. Schaefer, ${ }^{124}$

R. Schaefer, ${ }^{45}$ J. Schaeffer, ${ }^{86}$ S. Schaepe ${ }^{23}$ S. Schaetzel, ${ }^{60 b}$ U. Schäfer ${ }^{86}$ A. C. Schaffer, ${ }^{119}$ D. Schaile, ${ }^{102}$

R. D. Schamberger, ${ }^{150}$ V. A. Schegelsky, ${ }^{125}$ D. Scheirich, ${ }^{131}$ M. Schernau, ${ }^{166}$ C. Schiavi, ${ }^{53 a, 53 b}$ S. Schier, ${ }^{139}$

L. K. Schildgen, ${ }^{23}$ C. Schillo, ${ }^{51}$ M. Schioppa, ${ }^{40 a, 40 b}$ S. Schlenker, ${ }^{32}$ K. R. Schmidt-Sommerfeld, ${ }^{103}$ K. Schmieden, ${ }^{32}$ C. Schmitt, ${ }^{86}$ S. Schmitt, ${ }^{45}$ S. Schmitz, ${ }^{86}$ U. Schnoor, ${ }^{51}$ L. Schoeffel, ${ }^{138}$ A. Schoening, ${ }^{60 b}$ B. D. Schoenrock, ${ }^{93}$ E. Schopf,${ }^{23}$ M. Schott,${ }^{86}$ J. F. P. Schouwenberg, ${ }^{108}$ J. Schovancova, ${ }^{32}$ S. Schramm, ${ }^{52}$ N. Schuh, ${ }^{86}$ A. Schulte, ${ }^{86}$ M. J. Schultens, ${ }^{23}$

H.-C. Schultz-Coulon, ${ }^{60 a}$ H. Schulz ${ }^{17}$ M. Schumacher, ${ }^{51}$ B. A. Schumm, ${ }^{139}$ Ph. Schune, ${ }^{138}$ A. Schwartzman, ${ }^{145}$

T. A. Schwarz, ${ }^{92}$ H. Schweiger, ${ }^{87}$ Ph. Schwemling, ${ }^{138}$ R. Schwienhorst, ${ }^{93}$ J. Schwindling, ${ }^{138}$ A. Sciandra, ${ }^{23}$ G. Sciolla, ${ }^{25}$ M. Scornajenghi, ${ }^{40 a, 40 b}$ F. Scuri, ${ }^{126 a, 126 b}$ F. Scutti, ${ }^{91}$ J. Searcy, ${ }^{92}$ P. Seema, ${ }^{23}$ S. C. Seidel, ${ }^{107}$ A. Seiden, ${ }^{139}$ J. M. Seixas, ${ }^{26 a}$ G. Sekhniaidze, ${ }^{106 a}$ K. Sekhon, ${ }^{92}$ S. J. Sekula, ${ }^{43}$ N. Semprini-Cesari, ${ }^{22 a, 22 b}$ S. Senkin, ${ }^{37}$ C. Serfon, ${ }^{121}$ L. Serin, ${ }^{119}$

L. Serkin, ${ }^{167 a, 167 b}$ M. Sessa, ${ }^{136 a, 136 b}$ R. Seuster, ${ }^{172}$ H. Severini, ${ }^{115}$ T. Sfiligoj, ${ }^{78}$ F. Sforza, ${ }^{32}$ A. Sfyrla, ${ }^{52}$ E. Shabalina, ${ }^{57}$

N. W. Shaikh, ${ }^{148 a, 148 b}$ L. Y. Shan, ${ }^{35 a}$ R. Shang, ${ }^{169}$ J. T. Shank, ${ }^{24}$ M. Shapiro, ${ }^{16}$ P. B. Shatalov, ${ }^{99}$ K. Shaw, ${ }^{167 a, 167 b}$

S. M. Shaw ${ }^{87}$ A. Shcherbakova, ${ }^{148 a, 148 b}$ C. Y. Shehu, ${ }^{151}$ Y. Shen, ${ }^{115}$ N. Sherafati, ${ }^{31}$ P. Sherwood, ${ }^{81}$ L. Shi, ${ }^{153, p p}$ S. Shimizu, ${ }^{70}$ C. O. Shimmin, ${ }^{179}$ M. Shimojima, ${ }^{104}$ I. P. J. Shipsey, ${ }^{122}$ S. Shirabe, ${ }^{73}$ M. Shiyakova, ${ }^{68, q 9}$ J. Shlomi, ${ }^{175}$ A. Shmeleva, ${ }^{98}$ D. Shoaleh Saadi, ${ }^{97}$ M. J. Shochet, ${ }^{33}$ S. Shojaii, ${ }^{94 a}$ D. R. Shope, ${ }^{115}$ S. Shrestha, ${ }^{113}$ E. Shulga, ${ }^{100}$ M. A. Shupe, ${ }^{7}$ P. Sicho, ${ }^{129}$ A. M. Sickles, ${ }^{169}$ P. E. Sidebo, ${ }^{149}$ E. Sideras Haddad, ${ }^{147 c}$ O. Sidiropoulou,${ }^{177}$ A. Sidoti, ${ }^{22 a, 22 b}$ F. Siegert, ${ }^{47}$ Dj. Sijacki, ${ }^{14}$ J. Silva, ${ }^{128 a, 128 d}$ S. B. Silverstein, ${ }^{148 a}$ V. Simak, ${ }^{130}$ L. Simic, ${ }^{14}$ S. Simion, ${ }^{119}$ E. Simioni,${ }^{86}$ B. Simmons, ${ }^{81}$ M. Simon, ${ }^{86}$ P. Sinervo, ${ }^{161}$ N. B. Sinev, ${ }^{118}$ M. Sioli, ${ }^{22 a, 22 b}$ G. Siragusa, ${ }^{177}$ I. Siral,${ }^{92}$ S. Yu. Sivoklokov, ${ }^{101}$ J. Sjölin, ${ }^{148 a, 148 b}$ M. B. Skinner,${ }^{75}$ P. Skubic, ${ }^{115}$ M. Slater, ${ }^{19}$ T. Slavicek, ${ }^{130}$ M. Slawinska, ${ }^{42}$ K. Sliwa, ${ }^{165}$ R. Slovak, ${ }^{131}$ V. Smakhtin, ${ }^{175}$ B. H. Smart, ${ }^{5}$ J. Smiesko, ${ }^{146 a}$ N. Smirnov, ${ }^{100}$ S. Yu. Smirnov, ${ }^{100}$ Y. Smirnov, ${ }^{100}$ L. N. Smirnova, ${ }^{101, \mathrm{rr}}$ O. Smirnova, ${ }^{84}$ J. W. Smith, ${ }^{57}$ M. N. K. Smith, ${ }^{38}$ R. W. Smith, ${ }^{38}$ M. Smizanska, ${ }^{75}$ K. Smolek, ${ }^{130}$ A. A. Snesarev, ${ }^{98}$ I. M. Snyder, ${ }^{118}$ S. Snyder, ${ }^{27}$ R. Sobie,${ }^{172, p}$ F. Socher, ${ }^{47}$ A. Soffer, ${ }^{155}$ A. Søgaard,${ }^{49}$ D. A. Soh,${ }^{153}$ G. Sokhrannyi, ${ }^{78}$ C. A. Solans Sanchez,${ }^{32}$ M. Solar, ${ }^{130}$ E. Yu. Soldatov, ${ }^{100}$ U. Soldevila, ${ }^{170}$ A. A. Solodkov, ${ }^{132}$ A. Soloshenko, ${ }^{68}$ O. V. Solovyanov, ${ }^{132}$ V. Solovyev, ${ }^{125}$ P. Sommer, ${ }^{51}$ H. Son, ${ }^{165}$ A. Sopczak, ${ }^{130}$ D. Sosa, ${ }^{60 b}$ C. L. Sotiropoulou, ${ }^{126 a, 126 b}$ R. Soualah, ${ }^{167 a, 167 c}$ A. M. Soukharev, ${ }^{111, d}$ D. South, ${ }^{45}$

B. C. Sowden, ${ }^{80}$ S. Spagnolo, ${ }^{76 a, 76 b}$ M. Spalla, ${ }^{126 a, 126 b}$ M. Spangenberg, ${ }^{173}$ F. Spanò, ${ }^{80}$ D. Sperlich, ${ }^{17}$ F. Spettel,${ }^{103}$

T. M. Spieker, ${ }^{60}$ R. Spighi, ${ }^{22 a}$ G. Spigo, ${ }^{32}$ L. A. Spiller, ${ }^{91}$ M. Spousta, ${ }^{131}$ R. D. St. Denis, ${ }^{56, a}$ A. Stabile, ${ }^{94 a}$ R. Stamen, ${ }^{60 a}$ S. Stamm, ${ }^{17}$ E. Stanecka, ${ }^{42}$ R. W. Stanek, ${ }^{6}$ C. Stanescu, ${ }^{136 a}$ M. M. Stanitzki, ${ }^{45}$ B. S. Stapf, ${ }^{109}$ S. Stapnes, ${ }^{121}$

E. A. Starchenko, ${ }^{132}$ G. H. Stark, ${ }^{33}$ J. Stark, ${ }^{58}$ S. H Stark, ${ }^{39}$ P. Staroba, ${ }^{129}$ P. Starovoitov, ${ }^{60 a}$ S. Stärz, ${ }^{32}$ R. Staszewski, ${ }^{42}$ 
P. Steinberg, ${ }^{27}$ B. Stelzer, ${ }^{144}$ H. J. Stelzer, ${ }^{32}$ O. Stelzer-Chilton, ${ }^{163 a}$ H. Stenzel, ${ }^{55}$ G. A. Stewart ${ }^{56}$ M. C. Stockton, ${ }^{118}$ M. Stoebe ${ }^{90}$ G. Stoicea, ${ }^{28 b}$ P. Stolte,${ }^{57}$ S. Stonjek, ${ }^{103}$ A. R. Stradling, ${ }^{8}$ A. Straessner, ${ }^{47}$ M. E. Stramaglia, ${ }^{18}$ J. Strandberg, ${ }^{149}$ S. Strandberg, ${ }^{148 a, 148 b}$ M. Strauss, ${ }^{115}$ P. Strizenec, ${ }^{146 b}$ R. Ströhmer, ${ }^{177}$ D. M. Strom, ${ }^{118}$ R. Stroynowski, ${ }^{43}$ A. Strubig, ${ }^{49}$ S. A. Stucci, ${ }^{27}$ B. Stugu, ${ }^{15}$ N. A. Styles, ${ }^{45}$ D. Su, ${ }^{145}$ J. Su, ${ }^{127}$ S. Suchek,${ }^{60 a}$ Y. Sugaya, ${ }^{120}$ M. Suk,${ }^{130}$ V. V. Sulin, ${ }^{98}$ DMS Sultan, ${ }^{162 a, 162 b}$ S. Sultansoy, ${ }^{4 \mathrm{c}}$ T. Sumida, ${ }^{71}$ S. Sun, ${ }^{59}$ X. Sun, ${ }^{3}$ K. Suruliz, ${ }^{151}$ C. J. E. Suster, ${ }^{152}$ M. R. Sutton, ${ }^{151}$ S. Suzuki, ${ }^{69}$ M. Svatos, ${ }^{129}$ M. Swiatlowski, ${ }^{33}$ S. P. Swift, ${ }^{2}$ I. Sykora, ${ }^{146 a}$ T. Sykora,${ }^{131}$ D. Ta, ${ }^{51}$ K. Tackmann, ${ }^{45}$ J. Taenzer, ${ }^{155}$ A. Taffard, ${ }^{166}$ R. Tafirout, ${ }^{163 a}$ N. Taiblum,,${ }^{155}$ H. Takai, ${ }^{27}$ R. Takashima,${ }^{72}$ E. H. Takasugi, ${ }^{103}$ T. Takeshita,${ }^{142}$ Y. Takubo, ${ }^{69}$ M. Talby, ${ }^{88}$ A. A. Talyshev, ${ }^{11, d}$ J. Tanaka, ${ }^{157}$ M. Tanaka, ${ }^{159}$ R. Tanaka, ${ }^{119}$ S. Tanaka, ${ }^{69}$ R. Tanioka, ${ }^{70}$ B. B. Tannenwald,${ }^{113}$ S. Tapia Araya, ${ }^{34 \mathrm{~b}}$ S. Tapprogge ${ }^{86}$ S. Tarem, ${ }^{154}$ G. F. Tartarelli, ${ }^{94 a}$ P. Tas, ${ }^{131}$ M. Tasevsky, ${ }^{129}$ T. Tashiro, ${ }^{71}$ E. Tassi, ${ }^{40 a, 40 b}$ A. Tavares Delgado, ${ }^{128 a, 128 b}$ Y. Tayalati, ${ }^{137 \mathrm{e}}$ A. C. Taylor, ${ }^{107}$ G. N. Taylor, ${ }^{91}$ P. T. E. Taylor, ${ }^{91}$ W. Taylor, ${ }^{163 b}$

P. Teixeira-Dias, ${ }^{80}$ D. Temple, ${ }^{144}$ H. Ten Kate, ${ }^{32}$ P. K. Teng, ${ }^{153}$ J. J. Teoh, ${ }^{120}$ F. Tepel, ${ }^{178}$ S. Terada, ${ }^{69}$ K. Terashi, ${ }^{157}$

J. Terron, ${ }^{85}$ S. Terzo, ${ }^{13}$ M. Testa, ${ }^{50}$ R. J. Teuscher, ${ }^{161, \mathrm{p}}$ T. Theveneaux-Pelzer, ${ }^{88}$ F. Thiele, ${ }^{39}$ J. P. Thomas, ${ }^{19}$ J. Thomas-Wilsker, ${ }^{80}$ P. D. Thompson, ${ }^{19}$ A. S. Thompson,${ }^{56}$ L. A. Thomsen, ${ }^{179}$ E. Thomson, ${ }^{124}$ M. J. Tibbetts, ${ }^{16}$

R. E. Ticse Torres, ${ }^{88}$ V. O. Tikhomirov, ${ }^{98, s s}$ Yu. A. Tikhonov, ${ }^{11, d}$ S. Timoshenko, ${ }^{100}$ P. Tipton, ${ }^{179}$ S. Tisserant,${ }^{88}$

K. Todome ${ }^{159}$ S. Todorova-Nova, ${ }^{5}$ S. Todt, ${ }^{47}$ J. Tojo, ${ }^{73}$ S. Tokár, ${ }^{146 a}$ K. Tokushuku, ${ }^{69}$ E. Tolley, ${ }^{113}$ L. Tomlinson, ${ }^{87}$ M. Tomoto, ${ }^{105}$ L. Tompkins, ${ }^{145, t \mathrm{~K}} \mathrm{~K}$. Toms, ${ }^{107}$ B. Tong, ${ }^{59}$ P. Tornambe,${ }^{51}$ E. Torrence, ${ }^{118}$ H. Torres, ${ }^{144}$ E. Torró Pastor, ${ }^{140}$ J. Toth, ${ }^{88, \text { uu }}$ F. Touchard ${ }^{88}$ D. R. Tovey ${ }^{141}$ C. J. Treado, ${ }^{112}$ T. Trefzger, ${ }^{177}$ F. Tresoldi, ${ }^{151}$ A. Tricoli, ${ }^{27}$ I. M. Trigger, ${ }^{163 a}$ S. Trincaz-Duvoid, ${ }^{83}$ M. F. Tripiana, ${ }^{13}$ W. Trischuk, ${ }^{161}$ B. Trocmé,${ }^{58}$ A. Trofymov,${ }^{45}$ C. Troncon, ${ }^{94 a}$

M. Trottier-McDonald, ${ }^{16}$ M. Trovatelli, ${ }^{172}$ L. Truong, ${ }^{147 b}$ M. Trzebinski, ${ }^{42}$ A. Trzupek, ${ }^{42}$ K. W. Tsang, ${ }^{62 \mathrm{a}}$ J. C-L. Tseng, ${ }^{122}$ P. V. Tsiareshka, ${ }^{95}$ G. Tsipolitis, ${ }^{10}$ N. Tsirintanis, ${ }^{9}$ S. Tsiskaridze, ${ }^{13}$ V. Tsiskaridze,${ }^{51}$ E. G. Tskhadadze,${ }^{54 a}$ K. M. Tsui, ${ }^{62 a}$ I. I. Tsukerman, ${ }^{99}$ V. Tsulaia, ${ }^{16}$ S. Tsuno, ${ }^{69}$ D. Tsybychev, ${ }^{150} \mathrm{Y}$. Tu, ${ }^{62 \mathrm{~b}}$ A. Tudorache, ${ }^{28 \mathrm{~b}}$ V. Tudorache ${ }^{28 \mathrm{~b}}$ T. T. Tulbure, ${ }^{28 \mathrm{a}}$ A. N. Tuna ${ }^{59}$ S. A. Tupputi, ${ }^{22 a, 22 b}$ S. Turchikhin, ${ }^{68}$ D. Turgeman, ${ }^{175}$ I. Turk Cakir, ${ }^{4 b, v v}$ R. Turra,${ }^{94 a}$ P. M. Tuts, ${ }^{38}$ G. Ucchielli, ${ }^{22 a, 22 b}$ I. Ueda, ${ }^{69}$ M. Ughetto, ${ }^{148 a, 148 b}$ F. Ukegawa, ${ }^{164}$ G. Unal, ${ }^{32}$ A. Undrus, ${ }^{27}$ G. Unel, ${ }^{166}$ F. C. Ungaro, ${ }^{91}$ Y. Unno, ${ }^{69}$ C. Unverdorben, ${ }^{102}$ J. Urban, ${ }^{146 b}$ P. Urquijo, ${ }^{91}$ P. Urrejola, ${ }^{86}$ G. Usai,${ }^{8}$ J. Usui,${ }^{69}$ L. Vacavant ${ }^{88}$ V. Vacek, ${ }^{130}$ B. Vachon, ${ }^{90}$ K. O. H. Vadla, ${ }^{121}$ A. Vaidya, ${ }^{81}$ C. Valderanis, ${ }^{102}$ E. Valdes Santurio, ${ }^{148 a, 148 b}$ S. Valentinetti, ${ }^{22 a, 22 b}$ A. Valero, ${ }^{170}$ L. Valéry, ${ }^{13}$ S. Valkar, ${ }^{131}$ A. Vallier, ${ }^{5}$ J. A. Valls Ferrer, ${ }^{170}$ W. Van Den Wollenberg, ${ }^{109}$ H. van der Graaf, ${ }^{109}$ P. van Gemmeren, ${ }^{6}$ J. Van Nieuwkoop, ${ }^{144}$ I. van Vulpen, ${ }^{109}$ M. C. van Woerden, ${ }^{109}$ M. Vanadia, ${ }^{135 a, 135 b}$ W. Vandelli, ${ }^{32}$ A. Vaniachine, ${ }^{160}$ P. Vankov, ${ }^{109}$ G. Vardanyan, ${ }^{180}$ R. Vari, ${ }^{134 a}$ E. W. Varnes, ${ }^{7}$ C. Varni, ${ }^{53 a, 53 b}$ T. Varol, ${ }^{43}$ D. Varouchas, ${ }^{119}$ A. Vartapetian, ${ }^{8}$ K. E. Varvell, ${ }^{152}$ J. G. Vasquez, ${ }^{179}$ G. A. Vasquez, ${ }^{34 b}$ F. Vazeille, ${ }^{37}$ T. Vazquez Schroeder, ${ }^{90}$ J. Veatch, ${ }^{57}$ V. Veeraraghavan, ${ }^{7}$ L. M. Veloce, ${ }^{161}$ F. Veloso, ${ }^{128 a, 128 c}$ S. Veneziano, ${ }^{134 a}$ A. Ventura, ${ }^{76 a, 76 b}$ M. Venturi, ${ }^{172}$ N. Venturi, ${ }^{32}$ A. Venturini, ${ }^{25}$ V. Vercesi, ${ }^{123 a}$ M. Verducci, ${ }^{136 a, 136 \mathrm{~b}}$ W. Verkerke, ${ }^{109}$ A. T. Vermeulen, ${ }^{109}$ J. C. Vermeulen, ${ }^{109}$ M. C. Vetterli, ${ }^{144, e}$ N. Viaux Maira,${ }^{34 b}$ O. Viazlo,${ }^{84}$ I. Vichou, ${ }^{169, a}$ T. Vickey, ${ }^{141}$ O. E. Vickey Boeriu, ${ }^{141}$ G. H. A. Viehhauser, ${ }^{122}$ S. Viel, ${ }^{16}$ L. Vigani, ${ }^{122}$ M. Villa, ${ }^{22 a, 22 b}$ M. Villaplana Perez, ${ }^{94 a, 94 b}$ E. Vilucchi, ${ }^{50}$ M. G. Vincter, ${ }^{31}$ V. B. Vinogradov, ${ }^{68}$ A. Vishwakarma, ${ }^{45}$ C. Vittori, ${ }^{22 a, 22 b}$ I. Vivarelli, ${ }^{151}$ S. Vlachos, ${ }^{10}$ M. Vogel,${ }^{178}$ P. Vokac, ${ }^{130}$ G. Volpi, ${ }^{126 a, 126 b}$ H. von der Schmitt, ${ }^{103}$ E. von Toerne, ${ }^{23}$ V. Vorobel, ${ }^{131}$ K. Vorobev,${ }^{100}$ M. Vos, ${ }^{170}$ R. Voss, ${ }^{32}$ J. H. Vossebeld, ${ }^{77}$ N. Vranjes,${ }^{14}$ M. Vranjes Milosavljevic, ${ }^{14}$ V. Vrba, ${ }^{130}$ M. Vreeswijk, ${ }^{109}$ R. Vuillermet, ${ }^{32}$ I. Vukotic,${ }^{33}$ P. Wagner, ${ }^{23}$ W. Wagner, ${ }^{178}$ J. Wagner-Kuhr, ${ }^{102}$ H. Wahlberg, ${ }^{74}$ S. Wahrmund,${ }^{47}$ J. Wakabayashi, ${ }^{105}$ J. Walder ${ }^{75}$ R. Walker, ${ }^{102}$ W. Walkowiak, ${ }^{143}$ V. Wallangen, ${ }^{148 a, 148 b}$ C. Wang, ${ }^{35 b}$ C. Wang, ${ }^{36 b, w w}$ F. Wang, ${ }^{176} \mathrm{H}$. Wang, ${ }^{16} \mathrm{H}$. Wang, ${ }^{3}$ J. Wang, ${ }^{45}$ J. Wang, ${ }^{152}$ Q. Wang, ${ }^{115}$ R. Wang, ${ }^{6}$ S. M. Wang, ${ }^{153}$ T. Wang, ${ }^{38}$ W. Wang, ${ }^{153, x x}$ W. Wang, ${ }^{36 a, y y}$ Z. Wang, ${ }^{36 c}$ C. Wanotayaroj, ${ }^{18}$ A. Warburton, ${ }^{90}$ C. P. Ward, ${ }^{30}$ D. R. Wardrope, ${ }^{81}$ A. Washbrook ${ }^{49}$ P. M. Watkins, ${ }^{19}$ A. T. Watson, ${ }^{19}$ M. F. Watson, ${ }^{19}$ G. Watts, ${ }^{140}$ S. Watts,${ }^{87}$ B. M. Waugh, ${ }^{81}$ A. F. Webb, ${ }^{11}$ S. Webb, ${ }^{86}$ M. S. Weber, ${ }^{18}$ S. W. Weber, ${ }^{177}$ S. A. Weber, ${ }^{31}$ J. S. Webster, ${ }^{6}$ A. R. Weidberg, ${ }^{122}$ B. Weinert, ${ }^{64}$ J. Weingarten, ${ }^{57}$ M. Weirich, ${ }^{86}$ C. Weiser,${ }^{51}$ H. Weits, ${ }^{109}$ P. S. Wells, ${ }^{32}$ T. Wenaus, ${ }^{27}$ T. Wengler, ${ }^{32}$ S. Wenig, ${ }^{32}$ N. Wermes, ${ }^{23}$ M. D. Werner, ${ }^{67}$ P. Werner, ${ }^{32}$ M. Wessels, ${ }^{60 a}$

K. Whalen, ${ }^{118}$ N. L. Whallon, ${ }^{140}$ A. M. Wharton, ${ }^{75}$ A. S. White, ${ }^{92}$ A. White, ${ }^{8}$ M. J. White, ${ }^{1}$ R. White, ${ }^{34 b}$ D. Whiteson, ${ }^{166}$ B. W. Whitmore, ${ }^{75}$ F. J. Wickens, ${ }^{133}$ W. Wiedenmann, ${ }^{176}$ M. Wielers, ${ }^{133}$ C. Wiglesworth, ${ }^{39}$ L. A. M. Wiik-Fuchs,${ }^{51}$ A. Wildauer, ${ }^{103}$ F. Wilk, ${ }^{87}$ H. G. Wilkens, ${ }^{32}$ H. H. Williams, ${ }^{124}$ S. Williams,${ }^{109}$ C. Willis, ${ }^{93}$ S. Willocq, ${ }^{89}$ J. A. Wilson, ${ }^{19}$ I. Wingerter-Seez, ${ }^{5}$ E. Winkels, ${ }^{151}$ F. Winklmeier, ${ }^{118}$ O. J. Winston, ${ }^{151}$ B. T. Winter ${ }^{23}$ M. Wittgen, ${ }^{145}$ M. Wobisch, ${ }^{82, v}$ T. M. H. Wolf, ${ }^{109}$ R. Wolff, ${ }^{88}$ M. W. Wolter, ${ }^{42}$ H. Wolters, ${ }^{128 a, 128 c}$ V. W. S. Wong, ${ }^{171}$ S. D. Worm, ${ }^{19}$ B. K. Wosiek, ${ }^{42}$ J. Wotschack, ${ }^{32}$ K. W. Wozniak, ${ }^{42}$ M. Wu, ${ }^{33}$ S. L. Wu, ${ }^{176}$ X. Wu, ${ }^{52}$ Y. Wu, ${ }^{22}$ T. R. Wyatt, ${ }^{87}$ B. M. Wynne, ${ }^{49}$ S. Xella, ${ }^{39}$ 
Z. Xi ${ }^{92}$ L. Xia ${ }^{35 c}$ D. Xu, ${ }^{35 a}$ L. Xu, ${ }^{27}$ T. Xu ${ }^{138}$ B. Yabsley ${ }^{152}$ S. Yacoob,${ }^{147 a}$ D. Yamaguchi, ${ }^{159}$ Y. Yamaguchi, ${ }^{120}$ A. Yamamoto, ${ }^{69}$ S. Yamamoto, ${ }^{157}$ T. Yamanaka, ${ }^{157}$ M. Yamatani, ${ }^{157}$ K. Yamauchi, ${ }^{105}$ Y. Yamazaki, ${ }^{70}$ Z. Yan,${ }^{24}$ H. Yang, ${ }^{36 c}$ H. Yang, ${ }^{16}$ Y. Yang, ${ }^{153}$ Z. Yang, ${ }^{15}$ W-M. Yao, ${ }^{16}$ Y. C. Yap ${ }^{83}$ Y. Yasu, ${ }^{69}$ E. Yatsenko, ${ }^{5}$ K. H. Yau Wong, ${ }^{23}$ J. Ye, ${ }^{43}$ S. Ye, ${ }^{27}$ I. Yeletskikh, ${ }^{68}$ E. Yigitbasi, ${ }^{24}$ E. Yildirim,${ }^{86} \mathrm{~K}$. Yorita, ${ }^{174} \mathrm{~K}$. Yoshihara, ${ }^{124} \mathrm{C}$. Young, ${ }^{145}$ C. J. S. Young, ${ }^{32} \mathrm{~J}$. Yu, ${ }^{8} \mathrm{~J}$. Yu, ${ }^{67}$ S. P. Y. Yuen, ${ }^{23}$ I. Yusuff, ${ }^{30, z z}$ B. Zabinski, ${ }^{42}$ G. Zacharis, ${ }^{10}$ R. Zaidan, ${ }^{13}$ A. M. Zaitsev, ${ }^{132, m m}$ N. Zakharchuk, ${ }^{45}$ J. Zalieckas, ${ }^{15}$ A. Zaman, ${ }^{150}$ S. Zambito, ${ }^{59}$ D. Zanzi, ${ }^{91}$ C. Zeitnitz, ${ }^{178}$ G. Zemaityte, ${ }^{122}$ A. Zemla, ${ }^{41 a}$ J. C. Zeng, ${ }^{169}$ Q. Zeng, ${ }^{145}$ O. Zenin, ${ }^{132}$ T. Ženiš ${ }^{146 a}$ D. Zerwas, ${ }^{119}$ D. Zhang, ${ }^{92}$ F. Zhang, ${ }^{176}$ G. Zhang, ${ }^{36 a, y y}$ H. Zhang, ${ }^{35 b}$ J. Zhang, ${ }^{6}$ L. Zhang, ${ }^{51}$ L. Zhang, ${ }^{36 a}$ M. Zhang, ${ }^{169}$ P. Zhang, ${ }^{35 b}$ R. Zhang, ${ }^{23}$ R. Zhang, ${ }^{36, w w}$ X. Zhang, ${ }^{36 b}$ Y. Zhang, ${ }^{35 a, 35 d}$ Z. Zhang, ${ }^{119}$ X. Zhao, ${ }^{43}$ Y. Zhao, ${ }^{36 b, a a a}$ Z. Zhao, ${ }^{36 a}$ A. Zhemchugov, ${ }^{68}$ B. Zhou, ${ }^{92}$ C. Zhou, ${ }^{176}$ L. Zhou, ${ }^{43}$ M. Zhou, ${ }^{35 a, 35 d}$ M. Zhou, ${ }^{150}$ N. Zhou, ${ }^{35 \mathrm{c}}$ C. G. Zhu, ${ }^{36 \mathrm{~b}}$ H. Zhu, ${ }^{35 \mathrm{a}}$ J. Zhu, ${ }^{92}$ Y. Zhu, ${ }^{36 \mathrm{a}}$ X. Zhuang, ${ }^{35 \mathrm{a}}$ K. Zhukov, ${ }^{98}$ A. Zibell, ${ }^{177}$ D. Zieminska, ${ }^{64}$ N. I. Zimine, ${ }^{68}$ C. Zimmermann, ${ }^{86}$ S. Zimmermann, ${ }^{51}$ Z. Zinonos, ${ }^{103}$ M. Zinser, ${ }^{86}$ M. Ziolkowski, ${ }^{143}$ L. Živković, ${ }^{14}$ G. Zobernig, ${ }^{176}$ A. Zoccoli, ${ }^{22 a, 22 b}$ R. Zou, ${ }^{33}$ M. zur Nedden, ${ }^{17}$ and L. Zwalinski ${ }^{32}$

\title{
(ATLAS Collaboration)
}

\author{
${ }^{1}$ Department of Physics, University of Adelaide, Adelaide, Australia \\ ${ }^{2}$ Physics Department, SUNY Albany, Albany, New York, USA \\ ${ }^{3}$ Department of Physics, University of Alberta, Edmonton, Alberta, Canada \\ ${ }^{4 a}$ Department of Physics, Ankara University, Ankara, Turkey \\ ${ }^{4 b}$ Istanbul Aydin University, Istanbul, Turkey \\ ${ }^{4 \mathrm{c}}$ Division of Physics, TOBB University of Economics and Technology, Ankara, Turkey \\ ${ }^{5}$ LAPP, CNRS/IN2P3 and Université Savoie Mont Blanc, Annecy-le-Vieux, France \\ ${ }^{6}$ High Energy Physics Division, Argonne National Laboratory, Argonne, Illinois, USA \\ ${ }^{7}$ Department of Physics, University of Arizona, Tucson, Arizona, USA \\ ${ }^{8}$ Department of Physics, The University of Texas at Arlington, Arlington, Texas, USA \\ ${ }^{9}$ Physics Department, National and Kapodistrian University of Athens, Athens, Greece \\ ${ }^{10}$ Physics Department, National Technical University of Athens, Zografou, Greece \\ ${ }^{11}$ Department of Physics, The University of Texas at Austin, Austin, Texas, USA \\ ${ }^{12}$ Institute of Physics, Azerbaijan Academy of Sciences, Baku, Azerbaijan \\ ${ }^{13}$ Institut de Física d'Altes Energies (IFAE), The Barcelona Institute of Science and Technology, \\ Barcelona, Spain \\ ${ }^{14}$ Institute of Physics, University of Belgrade, Belgrade, Serbia \\ ${ }^{15}$ Department for Physics and Technology, University of Bergen, Bergen, Norway \\ ${ }^{16}$ Physics Division, Lawrence Berkeley National Laboratory and University of California, Berkeley, \\ California, USA \\ ${ }^{17}$ Department of Physics, Humboldt University, Berlin, Germany \\ ${ }^{18}$ Albert Einstein Center for Fundamental Physics and Laboratory for High Energy Physics, \\ University of Bern, Bern, Switzerland \\ ${ }^{19}$ School of Physics and Astronomy, University of Birmingham, Birmingham, United Kingdom \\ ${ }^{20 \mathrm{a}}$ Department of Physics, Bogazici University, Istanbul, Turkey \\ ${ }^{20 \mathrm{~b}}$ Department of Physics Engineering, Gaziantep University, Gaziantep, Turkey \\ ${ }^{20 \mathrm{~d}}$ Istanbul Bilgi University, Faculty of Engineering and Natural Sciences, Istanbul, Turkey \\ ${ }^{20 \mathrm{e}}$ Bahcesehir University, Faculty of Engineering and Natural Sciences, Istanbul, Turkey \\ ${ }^{21}$ Centro de Investigaciones, Universidad Antonio Narino, Bogota, Colombia \\ ${ }^{22 a}$ INFN Sezione di Bologna, Italy \\ ${ }^{22 \mathrm{~b}}$ Dipartimento di Fisica e Astronomia, Università di Bologna, Bologna, Italy \\ ${ }^{23}$ Physikalisches Institut, University of Bonn, Bonn, Germany \\ ${ }^{24}$ Department of Physics, Boston University, Boston, Massachusetts, USA \\ ${ }^{25}$ Department of Physics, Brandeis University, Waltham, Massachusetts, USA \\ ${ }^{26 a}$ Universidade Federal do Rio De Janeiro COPPE/EE/IF, Rio de Janeiro, Brazil \\ ${ }^{26 \mathrm{~b}}$ Electrical Circuits Department, Federal University of Juiz de Fora (UFJF), Juiz de Fora, Brazil \\ ${ }^{26 c}$ Federal University of Sao Joao del Rei (UFSJ), Sao Joao del Rei, Brazil \\ ${ }^{26 \mathrm{~d}}$ Instituto de Fisica, Universidade de Sao Paulo, Sao Paulo, Brazil \\ ${ }^{27}$ Physics Department, Brookhaven National Laboratory, Upton, New York, USA \\ ${ }^{28 a}$ Transilvania University of Brasov, Brasov, Romania \\ ${ }^{28 \mathrm{~b}}$ Horia Hulubei National Institute of Physics and Nuclear Engineering, Bucharest, Romania \\ ${ }^{28 c}$ Department of Physics, Alexandru Ioan Cuza University of Iasi, Iasi, Romania
}


${ }^{28 \mathrm{~d}}$ National Institute for Research and Development of Isotopic and Molecular Technologies, Physics Department, Cluj Napoca, Romania

${ }^{28 \mathrm{e}}$ University Politehnica Bucharest, Bucharest, Romania

${ }^{28 \mathrm{f}}$ West University in Timisoara, Timisoara, Romania

${ }^{29}$ Departamento de Física, Universidad de Buenos Aires, Buenos Aires, Argentina

${ }^{30}$ Cavendish Laboratory, University of Cambridge, Cambridge, United Kingdom

${ }^{31}$ Department of Physics, Carleton University, Ottawa ON, Canada

${ }^{32}$ CERN, Geneva, Switzerland

${ }^{33}$ Enrico Fermi Institute, University of Chicago, Chicago, Illinois, USA

${ }^{34 a}$ Departamento de Física, Pontificia Universidad Católica de Chile, Santiago, Chile

${ }^{34 \mathrm{~b}}$ Departamento de Física, Universidad Técnica Federico Santa María, Valparaíso, Chile

${ }^{35 a}$ Institute of High Energy Physics, Chinese Academy of Sciences, Beijing, China

${ }^{35 \mathrm{~b}}$ Department of Physics, Nanjing University, Jiangsu, China

${ }^{35 c}$ Physics Department, Tsinghua University, Beijing 100084, China

${ }^{35 \mathrm{~d}}$ University of Chinese Academy of Science (UCAS), Beijing, China

${ }^{36 a}$ Department of Modern Physics and State Key Laboratory of Particle Detection and Electronics, University of Science and Technology of China, Anhui, China

${ }^{36 \mathrm{~b}}$ School of Physics, Shandong University, Shandong, China

${ }^{36 c}$ Department of Physics and Astronomy, Key Laboratory for Particle Physics, Astrophysics and Cosmology, Ministry of Education; Shanghai Key Laboratory for Particle Physics and Cosmology, Shanghai Jiao Tong University, Shanghai(also at PKU-CHEP), China

${ }^{37}$ Université Clermont Auvergne, CNRS/IN2P3, LPC, Clermont-Ferrand, France

${ }^{38}$ Nevis Laboratory, Columbia University, Irvington, New York, USA

${ }^{39}$ Niels Bohr Institute, University of Copenhagen, Kobenhavn, Denmark

${ }^{40 a}$ INFN Gruppo Collegato di Cosenza, Laboratori Nazionali di Frascati, Italy

${ }^{40 \mathrm{~b}}$ Dipartimento di Fisica, Università della Calabria, Rende, Italy

${ }^{41 \mathrm{a}}$ AGH University of Science and Technology, Faculty of Physics and Applied Computer Science, Krakow, Poland

${ }^{41 \mathrm{~b}}$ Marian Smoluchowski Institute of Physics, Jagiellonian University, Krakow, Poland

${ }^{42}$ Institute of Nuclear Physics Polish Academy of Sciences, Krakow, Poland

${ }^{43}$ Physics Department, Southern Methodist University, Dallas, Texas, USA

${ }^{44}$ Physics Department, University of Texas at Dallas, Richardson, Texas, USA ${ }^{45}$ DESY, Hamburg and Zeuthen, Germany

${ }^{46}$ Lehrstuhl für Experimentelle Physik IV, Technische Universität Dortmund, Dortmund, Germany

${ }^{47}$ Institut für Kern- und Teilchenphysik, Technische Universität Dresden, Dresden, Germany

${ }^{48}$ Department of Physics, Duke University, Durham, North Carolina, USA

${ }^{49}$ SUPA - School of Physics and Astronomy, University of Edinburgh, Edinburgh, United Kingdom

${ }^{50}$ INFN e Laboratori Nazionali di Frascati, Frascati, Italy

${ }^{51}$ Fakultät für Mathematik und Physik, Albert-Ludwigs-Universität, Freiburg, Germany

${ }^{52}$ Departement de Physique Nucleaire et Corpusculaire, Université de Genève, Geneva, Switzerland

${ }^{53 a}$ INFN Sezione di GenovaGenova, Italy

${ }^{53 \mathrm{~b}}$ Dipartimento di Fisica, Università di Genova, Genova, Italy

${ }^{54 a}$ E. Andronikashvili Institute of Physics, Iv. Javakhishvili Tbilisi State University, Tbilisi, Georgia

${ }^{54 \mathrm{~b}}$ High Energy Physics Institute, Tbilisi State University, Tbilisi, Georgia

${ }^{55}$ II Physikalisches Institut, Justus-Liebig-Universität Giessen, Giessen, Germany

${ }^{56}$ SUPA - School of Physics and Astronomy, University of Glasgow, Glasgow, United Kingdom

${ }^{57}$ II Physikalisches Institut, Georg-August-Universität, Göttingen, Germany

${ }^{58}$ Laboratoire de Physique Subatomique et de Cosmologie, Université Grenoble-Alpes, CNRS/IN2P3, Grenoble, France

${ }^{59}$ Laboratory for Particle Physics and Cosmology, Harvard University, Cambridge, Massachusetts, USA

${ }^{60 \mathrm{a}}$ Kirchhoff-Institut für Physik, Ruprecht-Karls-Universität Heidelberg, Heidelberg, Germany

${ }^{60 \mathrm{~b}}$ Physikalisches Institut, Ruprecht-Karls-Universität Heidelberg, Heidelberg, Germany

${ }^{61}$ Faculty of Applied Information Science, Hiroshima Institute of Technology, Hiroshima, Japan

${ }^{62 \mathrm{a}}$ Department of Physics, The Chinese University of Hong Kong, Shatin, N.T., Hong Kong, China

${ }^{62 \mathrm{~b}}$ Department of Physics, The University of Hong Kong, Hong Kong, China

${ }^{62 \mathrm{c}}$ Department of Physics and Institute for Advanced Study, The Hong Kong University of Science and

Technology, Clear Water Bay, Kowloon, Hong Kong, China

${ }^{63}$ Department of Physics, National Tsing Hua University, Taiwan, Taiwan

${ }^{64}$ Department of Physics, Indiana University, Bloomington, Indiana, USA

${ }^{65}$ Institut für Astro- und Teilchenphysik, Leopold-Franzens-Universität, Innsbruck, Austria 
${ }^{66}$ University of Iowa, Iowa City, Iowa, USA

${ }^{67}$ Department of Physics and Astronomy, Iowa State University, Ames, Iowa, USA

${ }^{68}$ Joint Institute for Nuclear Research, JINR Dubna, Dubna, Russia

${ }^{69}$ KEK, High Energy Accelerator Research Organization, Tsukuba, Japan

${ }^{70}$ Graduate School of Science, Kobe University, Kobe, Japan

${ }^{71}$ Faculty of Science, Kyoto University, Kyoto, Japan

${ }^{72}$ Kyoto University of Education, Kyoto, Japan

${ }^{73}$ Research Center for Advanced Particle Physics and Department of Physics, Kyushu University, Fukuoka, Japan

${ }^{74}$ Instituto de Física La Plata, Universidad Nacional de La Plata and CONICET, La Plata, Argentina

${ }^{75}$ Physics Department, Lancaster University, Lancaster, United Kingdom

${ }^{76 a}$ INFN Sezione di Lecce, Italy

${ }^{76 b}$ Dipartimento di Matematica e Fisica, Università del Salento, Lecce, Italy

${ }^{77}$ Oliver Lodge Laboratory, University of Liverpool, Liverpool, United Kingdom

${ }^{78}$ Department of Experimental Particle Physics, Jožef Stefan Institute and Department of Physics, University of Ljubljana, Ljubljana, Slovenia

${ }^{79}$ School of Physics and Astronomy, Queen Mary University of London, London, United Kingdom

${ }^{80}$ Department of Physics, Royal Holloway University of London, Surrey, United Kingdom

${ }^{81}$ Department of Physics and Astronomy, University College London, London, United Kingdom

${ }^{82}$ Louisiana Tech University, Ruston, Los Angeles, USA

${ }^{83}$ Laboratoire de Physique Nucléaire et de Hautes Energies, UPMC and Université Paris-Diderot and CNRS/IN2P3, Paris, France

${ }^{84}$ Fysiska institutionen, Lunds universitet, Lund, Sweden

${ }^{85}$ Departamento de Fisica Teorica C-15, Universidad Autonoma de Madrid, Madrid, Spain

${ }^{86}$ Institut für Physik, Universität Mainz, Mainz, Germany

${ }^{87}$ School of Physics and Astronomy, University of Manchester, Manchester, United Kingdom

${ }^{88}$ CPPM, Aix-Marseille Université and CNRS/IN2P3, Marseille, France

${ }^{89}$ Department of Physics, University of Massachusetts, Amherst Massachusetts, USA

${ }^{90}$ Department of Physics, McGill University, Montreal QC, Canada

${ }^{91}$ School of Physics, University of Melbourne, Victoria, Australia

${ }^{92}$ Department of Physics, The University of Michigan, Ann Arbor, Michigan, USA

${ }^{93}$ Department of Physics and Astronomy, Michigan State University, East Lansing, Michigan, USA

${ }^{94 a}$ INFN Sezione di Milano, Milano, Italy

${ }^{94 \mathrm{~b}}$ Dipartimento di Fisica, Università di Milano, Milano, Italy

${ }^{95}$ B.I. Stepanov Institute of Physics, National Academy of Sciences of Belarus, Minsk, Republic of Belarus

${ }^{96}$ Research Institute for Nuclear Problems of Byelorussian State University, Minsk, Republic of Belarus

${ }^{97}$ Group of Particle Physics, University of Montreal, Montreal QC, Canada

${ }^{98}$ P.N. Lebedev Physical Institute of the Russian Academy of Sciences, Moscow, Russia

${ }^{99}$ Institute for Theoretical and Experimental Physics (ITEP), Moscow, Russia

${ }^{100}$ National Research Nuclear University MEPhI, Moscow, Russia

${ }^{101}$ D.V. Skobeltsyn Institute of Nuclear Physics, M.V. Lomonosov Moscow State University, Moscow, Russia

${ }^{102}$ Fakultät für Physik, Ludwig-Maximilians-Universität München, München, Germany

${ }^{103}$ Max-Planck-Institut für Physik (Werner-Heisenberg-Institut), München, Germany

${ }^{104}$ Nagasaki Institute of Applied Science, Nagasaki, Japan

${ }^{105}$ Graduate School of Science and Kobayashi-Maskawa Institute, Nagoya University, Nagoya, Japan

${ }^{106 a}$ INFN Sezione di Napoli, Italy

${ }^{106 \mathrm{~b}}$ Dipartimento di Fisica, Università di Napoli, Napoli, Italy

${ }^{107}$ Department of Physics and Astronomy, University of New Mexico, Albuquerque New Mexico, USA

${ }^{108}$ Institute for Mathematics, Astrophysics and Particle Physics, Radboud University Nijmegen/Nikhef, Nijmegen, Netherlands

${ }^{109}$ Nikhef National Institute for Subatomic Physics and University of Amsterdam, Amsterdam, Netherlands

${ }^{110}$ Department of Physics, Northern Illinois University, DeKalb, Illinois, USA

${ }^{111}$ Budker Institute of Nuclear Physics, SB RAS, Novosibirsk, Russia

${ }^{112}$ Department of Physics, New York University, New York, New York, USA

${ }^{113}$ Ohio State University, Columbus, Ohio, USA

${ }^{114}$ Faculty of Science, Okayama University, Okayama, Japan

${ }^{115}$ Homer L. Dodge Department of Physics and Astronomy, University of Oklahoma, Norman Oklahoma, USA

${ }^{116}$ Department of Physics, Oklahoma State University, Stillwater, Oklahoma, USA 
${ }^{117}$ Palacký University, RCPTM, Olomouc, Czech Republic

${ }^{118}$ Center for High Energy Physics, University of Oregon, Eugene, Oregon, USA

${ }^{119}$ LAL, Univ. Paris-Sud, CNRS/IN2P3, Université Paris-Saclay, Orsay, France

${ }^{120}$ Graduate School of Science, Osaka University, Osaka, Japan

${ }^{121}$ Department of Physics, University of Oslo, Oslo, Norway

${ }^{122}$ Department of Physics, Oxford University, Oxford, United Kingdom

${ }^{123 a}$ INFN Sezione di Pavia, Italy

${ }^{123 \mathrm{~b}}$ Dipartimento di Fisica, Università di Pavia, Pavia, Italy

${ }^{124}$ Department of Physics, University of Pennsylvania, Philadelphia Pennsylvania, USA

${ }^{125}$ National Research Centre "Kurchatov Institute" B.P.Konstantinov Petersburg Nuclear Physics Institute,

St. Petersburg, Russia

${ }^{126 a}$ INFN Sezione di Pisa, Italy

${ }^{126 b}$ Dipartimento di Fisica E. Fermi, Università di Pisa, Pisa, Italy

${ }^{127}$ Department of Physics and Astronomy, University of Pittsburgh, Pittsburgh, Pennsylvania, USA

${ }^{128 a}$ Laboratório de Instrumentação e Física Experimental de Partículas - LIP, Lisboa, Portugal

${ }^{128 \mathrm{~b}}$ Faculdade de Ciências, Universidade de Lisboa, Lisboa, Portugal

${ }^{128 c}$ Department of Physics, University of Coimbra, Coimbra, Portugal

${ }^{128 \mathrm{~d}}$ Centro de Física Nuclear da Universidade de Lisboa, Lisboa, Portugal

${ }^{128 \mathrm{e}}$ Departamento de Fisica, Universidade do Minho, Braga, Portugal

${ }^{128 \mathrm{f}}$ Departamento de Fisica Teorica y del Cosmos, Universidad de Granada, Granada, Portugal

${ }^{128 g}$ Dep Fisica and CEFITEC of Faculdade de Ciencias e Tecnologia, Universidade Nova de Lisboa, Caparica, Portugal

${ }^{129}$ Institute of Physics, Academy of Sciences of the Czech Republic, Praha, Czech Republic

${ }^{130}$ Czech Technical University in Prague, Praha, Czech Republic

${ }^{131}$ Charles University, Faculty of Mathematics and Physics, Prague, Czech Republic

${ }^{132}$ State Research Center Institute for High Energy Physics (Protvino), NRC KI, Russia

${ }^{133}$ Particle Physics Department, Rutherford Appleton Laboratory, Didcot, United Kingdom

${ }^{134 a}$ INFN Sezione di Roma, Italy

${ }^{134 b}$ Dipartimento di Fisica, Sapienza Università di Roma, Roma, Italy

${ }^{135 a}$ INFN Sezione di Roma Tor Vergata, Italy

${ }^{135 \mathrm{~b}}$ Dipartimento di Fisica, Università di Roma Tor Vergata, Roma, Italy

${ }^{136 a}$ INFN Sezione di Roma Tre, Italy

${ }^{136 \mathrm{~b}}$ Dipartimento di Matematica e Fisica, Università Roma Tre, Roma, Italy

${ }^{137 a}$ Faculté des Sciences Ain Chock, Réseau Universitaire de Physique des Hautes Energies Université Hassan II, Casablanca, Morocco

${ }^{137 \mathrm{~b}}$ Centre National de l'Energie des Sciences Techniques Nucleaires, Rabat, Morocco

${ }^{137 c}$ Faculté des Sciences Semlalia, Université Cadi Ayyad, LPHEA-Marrakech, Morocco

${ }^{137 \mathrm{~d}}$ Faculté des Sciences, Université Mohamed Premier and LPTPM, Oujda, Morocco

${ }^{137 \mathrm{e}}$ Faculté des sciences, Université Mohammed V, Rabat, Morocco

${ }^{138}$ DSM/IRFU (Institut de Recherches sur les Lois Fondamentales de l'Univers), CEA Saclay

(Commissariat à l'Energie Atomique et aux Energies Alternatives), Gif-sur-Yvette, France

${ }^{139}$ Santa Cruz Institute for Particle Physics, University of California Santa Cruz, Santa Cruz, California, USA

${ }^{140}$ Department of Physics, University of Washington, Seattle, Washington, USA

${ }^{141}$ Department of Physics and Astronomy, University of Sheffield, Sheffield, United Kingdom

${ }^{142}$ Department of Physics, Shinshu University, Nagano, Japan

${ }^{143}$ Department Physik, Universität Siegen, Siegen, Germany

${ }^{144}$ Department of Physics, Simon Fraser University, Burnaby BC, Canada

${ }^{145}$ SLAC National Accelerator Laboratory, Stanford, California, USA

${ }^{146 a}$ Faculty of Mathematics, Physics \& Informatics, Comenius University, Bratislava, Slovak Republic

${ }^{146 \mathrm{~b}}$ Department of Subnuclear Physics, Institute of Experimental Physics of the Slovak Academy of Sciences,

Kosice, Slovak Republic

${ }^{147 a}$ Department of Physics, University of Cape Town, Cape Town, South Africa

${ }^{147 \mathrm{~b}}$ Department of Physics, University of Johannesburg, Johannesburg, South Africa

${ }^{147 \mathrm{c}}$ School of Physics, University of the Witwatersrand, Johannesburg, South Africa

${ }^{148 a}$ Department of Physics, Stockholm University, Stockholm, Sweden

${ }^{148 \mathrm{~b}}$ The Oskar Klein Centre, Stockholm, Sweden

${ }^{149}$ Physics Department, Royal Institute of Technology, Stockholm, Sweden

${ }^{150}$ Departments of Physics and Astronomy and Chemistry, Stony Brook University,

Stony Brook, New York, USA 
${ }^{151}$ Department of Physics and Astronomy, University of Sussex, Brighton, United Kingdom

${ }^{152}$ School of Physics, University of Sydney, Sydney, Australia

${ }^{153}$ Institute of Physics, Academia Sinica, Taipei, Taiwan

${ }^{154}$ Department of Physics, Technion: Israel Institute of Technology, Haifa, Israel

${ }^{155}$ Raymond and Beverly Sackler School of Physics and Astronomy, Tel Aviv University, Tel Aviv, Israel

${ }^{156}$ Department of Physics, Aristotle University of Thessaloniki, Thessaloniki, Greece

${ }^{157}$ International Center for Elementary Particle Physics and Department of Physics, The University of Tokyo, Tokyo, Japan

${ }^{158}$ Graduate School of Science and Technology, Tokyo Metropolitan University, Tokyo, Japan

${ }^{159}$ Department of Physics, Tokyo Institute of Technology, Tokyo, Japan

${ }^{160}$ Tomsk State University, Tomsk, Russia

${ }^{161}$ Department of Physics, University of Toronto, Toronto ON, Canada ${ }^{162 \mathrm{a}}$ INFN-TIFPA, Trento, Italy

${ }^{162 \mathrm{~b}}$ University of Trento, Trento, Italy

${ }^{163 \mathrm{a}}$ TRIUMF, Vancouver BC, Canada

${ }^{163 \mathrm{~b}}$ Department of Physics and Astronomy, York University, Toronto ON, Canada

${ }^{164}$ Faculty of Pure and Applied Sciences, and Center for Integrated Research in Fundamental Science and Engineering, University of Tsukuba, Tsukuba, Japan

${ }^{165}$ Department of Physics and Astronomy, Tufts University, Medford, Massachusetts, USA

${ }^{166}$ Department of Physics and Astronomy, University of California Irvine, Irvine, California, USA

${ }^{167 a}$ INFN Gruppo Collegato di Udine, Sezione di Trieste, Udine, Italy

${ }^{167 \mathrm{~b}}$ ICTP, Trieste, Italy

${ }^{167 c}$ Dipartimento di Chimica, Fisica e Ambiente, Università di Udine, Udine, Italy

${ }^{168}$ Department of Physics and Astronomy, University of Uppsala, Uppsala, Sweden

${ }^{169}$ Department of Physics, University of Illinois, Urbana, Illinois, USA

${ }^{170}$ Instituto de Fisica Corpuscular (IFIC), Centro Mixto Universidad de Valencia - CSIC, Valencia, Spain

${ }^{171}$ Department of Physics, University of British Columbia, Vancouver BC, Canada

${ }^{172}$ Department of Physics and Astronomy, University of Victoria, Victoria BC, Canada

${ }^{173}$ Department of Physics, University of Warwick, Coventry, United Kingdom

${ }^{174}$ Waseda University, Tokyo, Japan

${ }^{175}$ Department of Particle Physics, The Weizmann Institute of Science, Rehovot, Israel

${ }^{176}$ Department of Physics, University of Wisconsin, Madison, Wisconsin, USA

${ }^{177}$ Fakultät für Physik und Astronomie, Julius-Maximilians-Universität, Würzburg, Germany

${ }^{178}$ Fakultät für Mathematik und Naturwissenschaften, Fachgruppe Physik, Bergische Universität Wuppertal, Wuppertal, Germany

${ }^{179}$ Department of Physics, Yale University, New Haven Connecticut, USA

${ }^{180}$ Yerevan Physics Institute, Yerevan, Armenia

${ }^{181}$ Centre de Calcul de l'Institut National de Physique Nucléaire et de Physique des Particules (IN2P3), Villeurbanne, France

${ }^{182}$ Academia Sinica Grid Computing, Institute of Physics, Academia Sinica, Taipei, Taiwan

${ }^{\mathrm{a}}$ Deceased.

${ }^{\mathrm{b}}$ Also at Department of Physics, King's College London, London, United Kingdom.

${ }^{c}$ Also at Institute of Physics, Azerbaijan Academy of Sciences, Baku, Azerbaijan.

${ }^{\mathrm{d}}$ Also at Novosibirsk State University, Novosibirsk, Russia.

${ }^{\mathrm{e}}$ Also at TRIUMF, Vancouver BC, Canada.

${ }^{\mathrm{f}}$ Also at Department of Physics and Astronomy, University of Louisville, Louisville, KY, USA.

${ }^{g}$ Also at Physics Department, An-Najah National University, Nablus, Palestine.

${ }^{\mathrm{h}}$ Also at Department of Physics, California State University, Fresno CA, USA.

${ }^{\mathrm{i}}$ Also at Department of Physics, University of Fribourg, Fribourg, Switzerland.

${ }^{\mathrm{j}}$ Also at II Physikalisches Institut, Georg-August-Universität, Göttingen, Germany.

${ }^{k}$ Also at Departament de Fisica de la Universitat Autonoma de Barcelona, Barcelona, Spain.

${ }^{1}$ Also at Departamento de Fisica e Astronomia, Faculdade de Ciencias, Universidade do Porto, Portugal.

${ }^{\mathrm{m}}$ Also at Tomsk State University, Tomsk, and Moscow Institute of Physics and Technology State University, Dolgoprudny, Russia.

${ }^{\mathrm{n}}$ Also at The Collaborative Innovation Center of Quantum Matter (CICQM), Beijing, China.

${ }^{\circ}$ Also at Universita di Napoli Parthenope, Napoli, Italy.

${ }^{\mathrm{p}}$ Also at Institute of Particle Physics (IPP), Canada.

${ }^{\mathrm{q}}$ Also at Horia Hulubei National Institute of Physics and Nuclear Engineering, Bucharest, Romania.

${ }^{\mathrm{r}}$ Also at Department of Physics, St. Petersburg State Polytechnical University, St. Petersburg, Russia.

${ }^{\mathrm{s}}$ Also at Borough of Manhattan Community College, City University of New York, New York City, USA. 
${ }^{t}$ Also at Department of Financial and Management Engineering, University of the Aegean, Chios, Greece.

${ }^{\mathrm{u}}$ Also at Centre for High Performance Computing, CSIR Campus, Rosebank, Cape Town, South Africa.

${ }^{\mathrm{v}}$ Also at Louisiana Tech University, Ruston LA, USA.

${ }^{\mathrm{w}}$ Also at Institucio Catalana de Recerca i Estudis Avancats, ICREA, Barcelona, Spain.

${ }^{\mathrm{x}}$ Also at Department of Physics, The University of Michigan, Ann Arbor MI, USA.

${ }^{y}$ Also at Graduate School of Science, Osaka University, Osaka, Japan.

${ }^{\mathrm{z}}$ Also at Fakultät für Mathematik und Physik, Albert-Ludwigs-Universität, Freiburg, Germany.

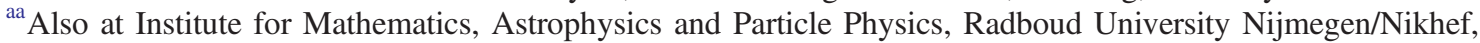
Nijmegen, Netherlands.

${ }^{\mathrm{bb}}$ Also at Department of Physics, The University of Texas at Austin, Austin TX, USA.

${ }^{c c}$ Also at Institute of Theoretical Physics, Ilia State University, Tbilisi, Georgia.

${ }^{\mathrm{dd}}$ Also at CERN, Geneva, Switzerland.

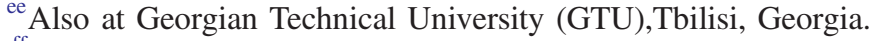

${ }^{\mathrm{ff}}$ Also at Ochadai Academic Production, Ochanomizu University, Tokyo, Japan.

${ }^{g g}$ Also at Manhattan College, New York, New York, USA.

${ }^{\text {hh }}$ Also at Departamento de Física, Pontificia Universidad Católica de Chile, Santiago, Chile.

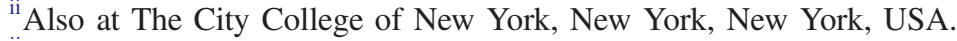

${ }^{\mathrm{jj}}$ Also at School of Physics, Shandong University, Shandong, China.

${ }^{k k}$ Also at Departamento de Fisica Teorica y del Cosmos, Universidad de Granada, Granada, Portugal.

${ }^{11}$ Also at Department of Physics, California State University, Sacramento CA, USA.

${ }^{\mathrm{mm}}$ Also at Moscow Institute of Physics and Technology State University, Dolgoprudny, Russia.

${ }^{n n}$ Also at Departement de Physique Nucleaire et Corpusculaire, Université de Genève, Geneva, Switzerland.

${ }^{\circ o}$ Also at Institut de Física d'Altes Energies (IFAE), The Barcelona Institute of Science and Technology, Barcelona, Spain.

${ }^{\mathrm{pp}}$ Also at School of Physics, Sun Yat-sen University, Guangzhou, China.

${ }^{\mathrm{qq}}$ Also at Institute for Nuclear Research and Nuclear Energy (INRNE) of the Bulgarian Academy of Sciences, Sofia, Bulgaria.

${ }^{\mathrm{rr}}$ Also at Faculty of Physics, M.V.Lomonosov Moscow State University, Moscow, Russia.

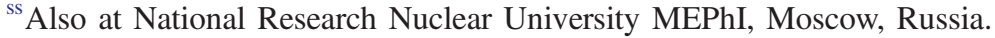

${ }^{\text {tt} A l s o ~ a t ~ D e p a r t m e n t ~ o f ~ P h y s i c s, ~ S t a n f o r d ~ U n i v e r s i t y, ~ S t a n f o r d ~ C A, ~ U S A . ~}$

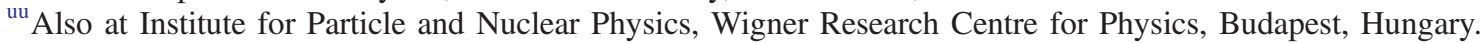

${ }^{\mathrm{v}}$ Also at Giresun University, Faculty of Engineering, Turkey.

${ }^{\text {ww }}$ Also at CPPM, Aix-Marseille Université and CNRS/IN2P3, Marseille, France.

${ }^{\mathrm{xx}}$ Also at Department of Physics, Nanjing University, Jiangsu, China.

${ }^{\text {yy }}$ Also at Institute of Physics, Academia Sinica, Taipei, Taiwan.

${ }^{\mathrm{zz}}$ Also at University of Malaya, Department of Physics, Kuala Lumpur, Malaysia.

${ }^{\text {aaa }}$ Also at LAL, Univ. Paris-Sud, CNRS/IN2P3, Université Paris-Saclay, Orsay, France. 